\title{
Determinantal Calabi-Yau varieties in Grassmannians and the Givental $I$-functions
}

\author{
Yoshinori Honma $^{a}$ Masahide Manabe $^{b}$ \\ ${ }^{a}$ Department of Current Legal Studies, Meiji Gakuin University, \\ Yokohama, Kanagawa 244-8539, Japan \\ ${ }^{b}$ School of Mathematics and Statistics, University of Melbourne, \\ Royal Parade, Parkville, VIC 3010, Australia \\ E-mail: yhonma@law.meijigakuin.ac.jp, masahidemanabe@gmail.com
}

ABStract: We examine a class of Calabi-Yau varieties of the determinantal type in Grassmannians and clarify what kind of examples can be constructed explicitly. We also demonstrate how to compute their genus-0 Gromov-Witten invariants from the analysis of the Givental $I$-functions. By constructing $I$-functions from the supersymmetric localization formula for the two dimensional gauged linear sigma models, we describe an algorithm to evaluate the genus-0 A-model correlation functions appropriately. We also check that our results for the Gromov-Witten invariants are consistent with previous results for known examples included in our construction.

KEywords: Topological Strings, Differential and Algebraic Geometry, Sigma Models

ARXiv EPrint: 1810.03685 


\section{Contents}

1 Introduction 1

2 Determinantal Calabi-Yau varieties in Grassmannians 2

2.1 Definitions 2

2.2 General dimensions 4

2.2.1 Ansatz (I) 4

2.2.2 Ansatz (II) 4

2.3 Determinantal Calabi-Yau 3-folds 5

2.3.1 $k=1 \quad 5$

$2.3 .2 k=2 \quad 6$

$\begin{array}{lll}2.3 .3 \quad k \geq 3 & 7\end{array}$

$3 \quad I$-functions and Gromov-Witten invariants $\quad 7$

3.1 Building blocks of $I$-functions

$\begin{array}{llr}3.2 & \text { Examples } & 9\end{array}$

3.2.1 Complete intersections in $\mathbb{P}^{n-1} \quad 9$

$\begin{array}{ll}\text { 3.2.2 Complete intersections in } G(k, n) & 11\end{array}$

$4 \quad$ I-functions of determinantal Calabi-Yau varieties $\quad \mathbf{1 5}$

$\begin{array}{ll}4.1 \text { I-functions and A-model correlators } & 16\end{array}$

$\begin{array}{ll}\text { 4.2 An algorithm to compute genus-0 invariants } & 17\end{array}$

$\begin{array}{lll}4.3 & \text { Illustrative examples of the computations } & 18\end{array}$

$\begin{array}{ll}\text { 4.3.1 Quintic family } & 18\end{array}$

4.3.2 Determinantal Calabi-Yau 3-folds in (2.23) 20

4.3.3 Determinantal Calabi-Yau 3-folds in (2.25) 20

5 Conclusions $\quad 24$

A Determinantal Calabi-Yau 2-folds and 4-folds 25

A.1 Determinantal Calabi-Yau 2-folds 25

$\begin{array}{ll}\text { A.1.1 } k=1 & 25\end{array}$

A.1.2 $k \geq 2 \quad 26$

A.2 Determinantal Calabi-Yau 4-folds 26

$\begin{array}{lll}\text { A.2.1 } k=1 & 26\end{array}$

$\begin{array}{lll}\text { A.2.2 } k=2 & 26\end{array}$

$\begin{array}{lll}\text { A. } 2.3 \quad k \geq 3 & 27\end{array}$

$\begin{array}{ll}\text { B Hodge number calculations via the Koszul complex } & 27\end{array}$

$\begin{array}{ll}\text { B.1 General algorithm } & 27\end{array}$

B.1.1 Step 1: computation of (B.2) 28

B.1.2 Step 2: computation of (B.1) 30 
B.2.1 Quintic Calabi-Yau 3-fold: $\mathcal{E}_{1}=\mathcal{O}_{V}(5)$ on $V=\mathbb{P}^{4}$

B.2.2 Grassmannian Calabi-Yau 3-fold: $\mathcal{E}_{3}=\mathcal{S}^{*}(1) \oplus \mathcal{O}_{V}(2)$ on $V=G(2,5) \quad 32$

B.2.3 Determinantal Calabi-Yau 3-fold in $(2.25)$ with $\mathcal{F}_{3}=\mathcal{S}^{*}(1) \oplus \mathcal{O}_{V}(1) \quad 34$

C Genus-0 invariants of determinantal Calabi-Yau 4-folds 36

$\begin{array}{lll}\text { C.1 Sextic family } & 36\end{array}$

C.2 Determinantal Calabi-Yau 4-folds in (A.8) 41

\section{Introduction}

String compactifications to lower dimensions preserving supersymmetry motivate us to study Calabi-Yau varieties as initiated in [1]. To analyze various properties of CalabiYau backgrounds, it is useful to consider the two dimensional gauged linear sigma model (GLSM) [2]. This model corresponds to the UV description of the non-linear sigma models on Calabi-Yau backgrounds and has provided a powerful technique to compute the correlation functions exactly (see for example [3]). Utilizing the duality property of the model, a physical understanding about mirror symmetry has also been developed [4] (see also [5, 6] for several recent developments).

On the other hand, by using the supersymmetric localization techniques [7], exact formula for the GLSM partition functions $[8,9]$ (see also [10, 11]) and correlation functions $[12,13]$ on the 2 -sphere backgrounds has been clarified. This means that one can evaluate the genus-0 Gromov-Witten invariants from the GLSM calculation in a direct fashion. This methodology has also been applied to the GLSMs with non-abelian gauge groups and the study of the complete intersection Calabi-Yau varieties in Grassmannians has progressed in the last few years $[14,15]$. Several notable aspects of the GLSM correlation functions have also been clarified in $[16,17]$.

While the toric complete intersection varieties described by abelian GLSMs have been thoroughly investigated in various contexts, a comprehensive understanding about the noncomplete intersection varieties requires further efforts. In [18], as an example of a class of non-complete intersections, the determinantal varieties $[19,20]$ and the associated nonabelian GLSMs have been investigated. The aim of our work is to make advances in the study of the determinantal varieties and provides a further step toward the comprehensive understanding of general Calabi-Yau backgrounds with non-abelian GLSM descriptions.

In this paper we explicitly clarify what kind of determinantal Calabi-Yau varieties in Grassmannians can be constructed while satisfying several requirements. We mainly focus on the 3-fold examples and the analysis for the determinantal Calabi-Yau 2-folds and 4-folds is summarized in appendix. We will also study the quantum aspects of the GLSM associated with determinantal varieties. Our method is based on the analysis of the so-called Givental $I$-functions [21-23] which can be extracted from the localization formula for the GLSM on a supersymmetric 2-sphere. In particular, we will compute 
the genus-0 Gromov-Witten invariants of determinantal Calabi-Yau varieties by using a conjectural handy formula, and check that our results coincide with previous results for known examples included in our classification.

This paper is organized as follows. First we examine a class of determinantal CalabiYau varieties in Grassmannians satisfying several requirements and specify the possible examples in section 2. In section 3 , we briefly review the computation of the genus- 0 invariants of complete intersection Calabi-Yau varieties in complex projective spaces and Grassmannians utilizing the $I$-functions, and provide a conjectural formula for Grassmannian Calabi-Yau varieties. In section 4, we compute the genus-0 invariants of determinantal Calabi-Yau varieties with GLSM realizations by using the algorithm described in section 3. Section 5 is devoted to conclusions and discussions. In appendix A we summarize our results of the analysis for determinantal Calabi-Yau 2-folds and 4-folds. In appendix B, we take a brief look at the computation of Hodge numbers using the Koszul complex and demonstrate several computations explicitly. In appendix $\mathrm{C}$ we summarize the data of the genus-0 invariants of several determinantal Calabi-Yau 4-folds.

\section{Determinantal Calabi-Yau varieties in Grassmannians}

In this section, we first review a minimal ingredient of determinantal varieties, following [18] (see also $[19,20]$ ). Afterwards we classify a class of determinantal Calabi-Yau 3-folds in Grassmannians satisfying appropriate conditions.

\subsection{Definitions}

Let $V$ be a compact algebraic variety, and $A: \mathcal{E}_{p} \rightarrow \mathcal{F}_{q}$ be a linear map from a rank $p$ vector bundle $\mathcal{E}_{p}$ on $V$ to a rank $q$ vector bundle $\mathcal{F}_{q}$ on $V$. Here we assume that the linear map $A$ is a global holomorphic section of the rank $p q$-bundle $\operatorname{Hom}\left(\mathcal{E}_{p}, \mathcal{F}_{q}\right) \cong \mathcal{E}_{p}^{*} \otimes \mathcal{F}_{q}$ with maximal rank at a generic point of $V$. By representing the linear map $A$ locally as a $q \times p$ matrix $A(\phi)$ of the holomorphic sections, a determinantal variety is defined as

$$
Z(A, \ell)=\{\phi \in V \mid \operatorname{rank} A(\phi) \leq \ell\}, \quad 0 \leq \ell<\min (p, q),
$$

where $\phi$ denotes the homogeneous coordinates on $V$. The complex codimension of $Z(A, \ell)$ in $V$ is given by

$$
\operatorname{codim} Z(A, \ell)=(p-\ell)(q-\ell)
$$

Here $(\ell+1) \times(\ell+1)$ minors of $A(\phi)$ generate the ideal $I(Z(A, \ell))$. Since $\operatorname{codim} Z(A, \ell)=$ $(p-\ell)(q-\ell)<\left(\begin{array}{c}p \\ \ell+1\end{array}\right)\left(\begin{array}{c}q \\ \ell+1\end{array}\right)$ for $\ell \geq 1$, the ideal $I(Z(A, \ell))$ has non-trivial relations called syzygies and the determinantal variety $Z(A, \ell)$ for $\ell \geq 1$ is not a complete intersection. As argued in [18], a simple analysis of the Jacobian matrix implies that $Z(A, \ell)$ for $\ell \geq 1$ has singular loci along $Z(A, \ell-1) \subset Z(A, \ell)$ only. One can resolve these singularities by the so-called incidence correspondence [18, 19],

$$
X_{A}^{V}=\left\{(\phi, x) \in V_{\mathcal{E}_{p}, p-\ell} \mid A(\phi) x=0\right\} \longrightarrow Z(A, \ell)
$$


where $V_{\mathcal{E}_{p}, p-\ell}$ denotes the fibration

$$
G\left(p-\ell, \mathcal{E}_{p}\right) \longrightarrow V_{\mathcal{E}_{p}, p-\ell} \stackrel{\pi}{\longrightarrow} V
$$

with Grassmannian fibers $G\left(p-\ell, \mathcal{E}_{p}\right)$ of $(p-\ell)$-planes with respect to the $p$-dimensional fibers of $\mathcal{E}_{p}$. It is worth noting that the codimension of the singular loci in $V$ is $\operatorname{codim} Z(A, \ell-1)=\operatorname{codim} Z(A, \ell)+p+q-2 \ell+1$, and then the determinantal variety $Z(A, \ell)$ with the dimension less than $p+q-2 \ell+1$ does not have singular loci [18]. ${ }^{1}$

Remark $2.1([18])$. Since $\ell<\min (p, q)$, the determinantal varieties with dimension less than 2 do not have singular loci. The determinantal 3 -folds have singular points only when $p=q=\ell+1$. The determinantal 4 -folds have singular lines only when $p=q=\ell+1$, and have singular points only when $(p, q)=(\ell+1, \ell+2)$ or $(p, q)=(\ell+2, \ell+1)$.

In this paper we only consider the square $(p=q)$ determinantal varieties with

$$
V=G(k, n), \quad \mathcal{E}_{p}=\mathcal{O}_{V}^{\oplus p}, \quad \operatorname{rank} \mathcal{F}_{p}=p,
$$

where $G(k, n)$ is the complex Grassmannian defined by the set of $k$-planes in $\mathbb{C}^{n}$, and $\mathcal{O}_{V}$ is the structure sheaf of $V$. Then the variety $V_{\mathcal{E}_{p}, p-\ell}$ can be described by a product variety $V_{\mathcal{E}_{p}, p-\ell} \cong G(k, n) \times G(p-\ell, p)$ and the incidence correspondence (2.3) becomes

$$
X_{A}:=X_{A}^{G(k, n)}=\left\{(\phi, x) \in G(k, n) \times G\left(\ell_{p}^{\vee}, p\right) \mid A(\phi) x=0\right\}, \quad \ell_{p}^{\vee}:=p-\ell .
$$

In addition we require $n=\ell_{p}^{\vee} \mathfrak{c}_{1}\left(\mathcal{F}_{p}\right)$ derived from Calabi-Yau condition [18]. Here

$$
c_{1}\left(\mathcal{F}_{p}\right)=\mathfrak{c}_{1}\left(\mathcal{F}_{p}\right) \sigma_{1}
$$

is the first Chern class of $\mathcal{F}_{p}$ and $\sigma_{1}=c_{1}(\mathcal{Q})$ is the Schubert class of $G(k, n)$. $\mathcal{Q}$ is the universal quotient bundle on $G(k, n)$. The dimension of $X_{A}$ is given by $\operatorname{dim} X_{A}=$ $k(n-k)-(p-\ell)^{2}=\ell_{p}^{\vee}\left(k \mathfrak{c}_{1}\left(\mathcal{F}_{p}\right)-\ell_{p}^{\vee}\right)-k^{2}$. By taking the duality $G(k, n) \cong G(n-k, n)$ into consideration, here we only consider the case with $2 k \leq n$. Furthermore, the rank condition $0 \leq \ell<p$ can be rephrased as $0<\ell_{p}^{\vee} \leq p=\operatorname{rank} \mathcal{F}_{p}$.

In summary, we have seen that the following conditions ${ }^{2}$ must be satisfied in order to realize the determinantal varieties appropriately.

$$
\begin{array}{lll}
\text { 1. } & \text { Dimensional condition: } & \ell_{p}^{\vee}\left(k \mathfrak{c}_{1}\left(\mathcal{F}_{p}\right)-\ell_{p}^{\vee}\right)=k^{2}+\operatorname{dim} X_{A} . \\
\text { 2. } & \text { Calabi-Yau condition: } & n=\ell_{p}^{\vee} \mathfrak{c}_{1}\left(\mathcal{F}_{p}\right) . \\
\text { 3. } & \text { Duality condition: } & 2 k \leq n . \\
\text { 4. } & \text { Rank condition: } & 0<\ell_{p}^{\vee} \leq p=\operatorname{rank} \mathcal{F}_{p} .
\end{array}
$$

In the following, we will classify the determinantal Calabi-Yau 3-folds satisfying the above four conditions. Although we consider the desingularized determinantal varieties $X_{A}$, the following analysis also gives a classification of $Z(A, \ell) .^{3}$

\footnotetext{
${ }^{1}$ As noted in [18], one can also use the incidence correspondence (2.3) to describe the determinantal varieties without singular loci.

${ }^{2}$ Note that we do not impose irreducibility or the conditions $H^{i}\left(X_{A}, \mathcal{O}_{X_{A}}\right)=0$ in our analysis.

${ }^{3}$ See appendix A for the analysis of determinantal Calabi-Yau 2-folds and 4-folds.
} 


\subsection{General dimensions}

Before moving on to the discussion about the determinantal Calabi-Yau 3-folds, let us consider general implications of the above requirements. In general dimensions, obviously the following two ansatz always satisfy the dimensional condition (2.7).

$$
\begin{array}{lll}
\text { Ansatz (I) : } & \left(\ell_{p}^{\vee}, k \mathfrak{c}_{1}\left(\mathcal{F}_{p}\right)\right)=\left(1, \operatorname{dim} X_{A}+k^{2}+1\right), \\
\text { Ansatz (II) : } & \left(\ell_{p}^{\vee}, k \mathfrak{c}_{1}\left(\mathcal{F}_{p}\right)\right)=\left(\operatorname{dim} X_{A}+k^{2}, \operatorname{dim} X_{A}+k^{2}+1\right) .
\end{array}
$$

In the following, we will illustrate what kind of setups for $\left(k, n ; \ell_{p}^{\vee}, \mathfrak{c}_{1}\left(\mathcal{F}_{p}\right)\right)$ satisfy all the above requirements if we start from the Ansatz (I) or (II). ${ }^{4}$

\subsubsection{Ansatz (I)}

In this case, the Calabi-Yau condition (2.8) becomes

$$
n=\frac{1}{k}\left(\operatorname{dim} X_{A}+k^{2}+1\right) .
$$

Then the duality condition (2.9) implies

$$
k^{2} \leq \operatorname{dim} X_{A}+1
$$

which means that examples with $k \geq 3$ provide determinantal varieties with $\operatorname{dim} X_{A} \geq 8$.

When $k=1, V$ is given by $G(1, n) \cong \mathbb{P}^{n-1}$ and one obtains the solutions with

$$
\left(k, n ; \ell_{p}^{\vee}, \mathfrak{c}_{1}\left(\mathcal{F}_{p}\right)\right)=\left(1, \operatorname{dim} X_{A}+2 ; 1, \operatorname{dim} X_{A}+2\right) .
$$

In this case the rank condition (2.10) is trivially satisfied, and appropriate $\mathcal{F}_{p}$ on $V$ are given by the following vector bundles associated with the integer partitions of $\operatorname{dim} X_{A}+2$ :

$$
\mathcal{F}_{p}=\oplus_{i=1}^{r} \mathcal{O}_{V}\left(p_{i}\right), \quad p_{1} \geq p_{2} \geq \cdots \geq p_{r}>0, \quad \sum_{i=1}^{r} p_{i}=\operatorname{dim} X_{A}+2 .
$$

When $k=2, V$ becomes $G(2, n)$ and one finds the solutions with

$$
\left(k, n ; \ell_{p}^{\vee}, \mathfrak{c}_{1}\left(\mathcal{F}_{p}\right)\right)=\left(2,\left(\operatorname{dim} X_{A}+5\right) / 2 ; 1,\left(\operatorname{dim} X_{A}+5\right) / 2\right) .
$$

Since $n$ has to be an integer, this type of solution can exist only when the dimension of $X_{A}$ is odd. Moreover, (2.14) requires $\operatorname{dim} X_{A} \geq 3$.

\subsubsection{Ansatz (II)}

In this case, the Calabi-Yau condition (2.8) becomes

$$
n=\frac{1}{k}\left(\operatorname{dim} X_{A}+k^{2}\right)\left(\operatorname{dim} X_{A}+k^{2}+1\right),
$$

\footnotetext{
${ }^{4}$ Of course there exist other solutions which do not belong to the Ansatz (I) or (II). In section 2.3 we have also taken into account this kind of solutions and checked, by Mathematica and Maple, that our result exhausted all the possible solutions up to $k=50$.
} 
and the duality condition (2.9) is trivially satisfied. Thus we only need to consider the rank condition given by

$$
\operatorname{dim} X_{A}+k^{2} \leq \operatorname{rank} \mathcal{F}_{p}
$$

For example, when $k=1$, we obtain the following solutions

$$
\begin{aligned}
& \left(k, n ; \ell_{p}^{\vee}, \mathfrak{c}_{1}\left(\mathcal{F}_{p}\right)\right)=\left(1,\left(\operatorname{dim} X_{A}+1\right)\left(\operatorname{dim} X_{A}+2\right) ; \operatorname{dim} X_{A}+1, \operatorname{dim} X_{A}+2\right) \\
& \text { with } \mathcal{F}_{p}=\mathcal{O}_{V}(1)^{\oplus \operatorname{dim} X_{A}} \oplus \mathcal{O}_{V}(2), \mathcal{O}_{V}(1)^{\oplus\left(\operatorname{dim} X_{A}+2\right)} .
\end{aligned}
$$

Note that the rank condition (2.19) strongly constrain the possible vector bundles.

\subsection{Determinantal Calabi-Yau 3-folds}

Here we will focus on the square determinantal Calabi-Yau 3-folds and determine what kind of setups satisfy the above four conditions. Let us start with the dimensional condition given by

$$
\ell_{p}^{\vee}\left(k \mathfrak{c}_{1}\left(\mathcal{F}_{p}\right)-\ell_{p}^{\vee}\right)=k^{2}+3
$$

Then we will find out which type of choices for $\left(k, n ; \ell_{p}^{\vee}, \mathfrak{c}_{1}\left(\mathcal{F}_{p}\right)\right)$ can be possible while changing the parameter $k$.

\subsection{1 $k=1$}

In this case we have $V=G(1, n) \cong \mathbb{P}^{n-1}$. From (2.15) and (2.16) one finds that there exists a "quintic family" (see for example [24]) given by

$$
\left(k, n ; \ell_{p}^{\vee}, \mathfrak{c}_{1}\left(\mathcal{F}_{p}\right)\right)=(1,5 ; 1,5) \text { with } \mathcal{F}_{p}=\oplus_{i=1}^{r} \mathcal{O}_{V}\left(p_{i}\right), p_{1} \geq p_{2} \geq \cdots \geq p_{r}>0, \sum_{i=1}^{r} p_{i}=5
$$

The example constructed from $\mathcal{F}_{p}=\mathcal{O}_{V}(5)$ with $p=1$ (i.e. $\ell=0$ ) is the well-known quintic Calabi-Yau 3-fold, which is the zero locus of a holomorphic section of $\mathcal{O}_{\mathbb{P}^{4}}(5)$.

Since $\ell_{p}^{\vee}=1$ (i.e. $p=\ell+1$ ), according to the Remark 2.1 , generically the above quintic families have singular points. The determinantal Calabi-Yau 3-folds in this class are connected by the deformations of complex structures, and it is known that the desingularized 3 -folds are related by the so-called extremal transitions. ${ }^{5}$

Apart from the above quintic family, one can also find the following solutions

$$
\begin{aligned}
\left(k, n ; \ell_{p}^{\vee}, \mathfrak{c}_{1}\left(\mathcal{F}_{p}\right)\right) & =(1,8 ; 2,4) \text { with } \\
\mathcal{F}_{p} & =\mathcal{O}_{V}(1) \oplus \mathcal{O}_{V}(3), \mathcal{O}_{V}(2)^{\oplus 2}, \mathcal{O}_{V}(1)^{\oplus 2} \oplus \mathcal{O}_{V}(2), \mathcal{O}_{V}(1)^{\oplus 4}
\end{aligned}
$$

Here the first two examples with $p=2$ (i.e. $\ell=0$ ) in $(2.23)$ can be identified with the well-known complete intersection Calabi-Yau 3-folds as

$$
X_{A} \text { with } \mathcal{O}_{V}(1) \oplus \mathcal{O}_{V}(3) \longleftrightarrow X_{3,3} \subset \mathbb{P}^{5}, \quad X_{A} \text { with } \mathcal{O}_{V}(2)^{\oplus 2} \longleftrightarrow X_{2,2,2,2} \subset \mathbb{P}^{7}
$$

\footnotetext{
${ }^{5}$ The comparison of topological invariants in section 4.3.1 makes this point clearly understandable.
} 
where $X_{d_{1}, \ldots, d_{r}} \subset \mathbb{P}^{n-1}$ denotes the complete intersection variety defined by the zero locus of a holomorphic section of the vector bundle $\oplus_{a=1}^{r} \mathcal{O}_{\mathbb{P}^{n-1}}\left(d_{a}\right)$. The last two examples in (2.23) are Gulliksen-Negård type 3-folds studied in [25].

Moreover, (2.20) provides another type of solutions given by

$$
\left(k, n ; \ell_{p}^{\vee}, \mathfrak{c}_{1}\left(\mathcal{F}_{p}\right)\right)=(1,20 ; 4,5) \text { with } \mathcal{F}_{p}=\mathcal{O}_{V}(1)^{\oplus 3} \oplus \mathcal{O}_{V}(2), \mathcal{O}_{V}(1)^{\oplus 5}
$$

The Calabi-Yau 3-fold $X_{A}$ in (2.24) constructed from $\mathcal{F}_{p}=\mathcal{O}_{V}(1)^{\oplus 3} \oplus \mathcal{O}_{V}(2)$ with $p=4$ (i.e. $\ell=0$ ) has the following isomorphism:

$$
X_{A} \text { with } \mathcal{O}_{V}(1)^{\oplus 3} \oplus \mathcal{O}_{V}(2) \longleftrightarrow X_{2,2,2,2} \subset \mathbb{P}^{7}
$$

The other example constructed from $\mathcal{F}_{p}=\mathcal{O}_{V}(1)^{\oplus 5}$ has been studied in [18].

\subsection{2 $k=2$}

In this case, $V$ becomes the Grassmannians $V=G(2, n)$. Compared with the complex projective spaces, there exist additional components for the vector bundles on the Grassmannians, as explained in the followings.

When $k \geq 2$, beside the line bundle $\mathcal{O}_{V}(d)$ on $V=G(k, n)$, one can also consider vector bundles with rank greater than one denoted by

$$
\mathcal{S}^{*} \text { and } \mathcal{Q} \text {. }
$$

These are known as the dual of the universal subbundle and the universal quotient bundle on $G(k, n)$, respectively. Note that they fulfill the relation $\wedge^{k} \mathcal{S}^{*} \cong \mathcal{O}_{V}(1)$ and satisfy

$$
\operatorname{rank} \mathcal{S}^{*}=k, \quad \mathfrak{c}_{1}\left(\mathcal{S}^{*}\right)=1, \quad \operatorname{rank} \mathcal{Q}=n-k, \quad \mathfrak{c}_{1}(\mathcal{Q})=1 .
$$

Accordingly, general irreducible vector bundles can be constructed as

$$
\begin{array}{rlrl}
\operatorname{Sym}^{m} \mathcal{S}^{*}(d) & :=\operatorname{Sym}^{m} \mathcal{S}^{*} \otimes \mathcal{O}_{V}(d), & & \wedge^{m} \mathcal{S}^{*}(d):=\wedge^{m} \mathcal{S}^{*} \otimes \mathcal{O}_{V}(d), \\
\operatorname{Sym}^{m} \mathcal{Q}(d):=\operatorname{Sym}^{m} \mathcal{Q}^{*} \otimes \mathcal{O}_{V}(d), & \wedge^{m} \mathcal{Q}(d):=\wedge^{m} \mathcal{Q}^{*} \otimes \mathcal{O}_{V}(d),
\end{array}
$$

where

$$
\begin{array}{rlrl}
\operatorname{rank} \operatorname{Sym}^{m} \mathcal{S}^{*}(d) & =\left(\begin{array}{c}
k+m-1 \\
m
\end{array}\right), & \mathfrak{c}_{1}\left(\operatorname{Sym}^{m} \mathcal{S}^{*}(d)\right) & =\left(\begin{array}{c}
k+m-1 \\
k
\end{array}\right)+d\left(\begin{array}{c}
k+m-1 \\
m
\end{array}\right), \\
\operatorname{rank} \wedge^{m} \mathcal{S}^{*}(d) & =\left(\begin{array}{c}
k \\
m
\end{array}\right), \\
\mathfrak{c}_{1}\left(\wedge^{m} \mathcal{S}^{*}(d)\right) & =\left(\begin{array}{c}
k-1 \\
m-1
\end{array}\right)+d\left(\begin{array}{c}
k \\
m
\end{array}\right), \\
\operatorname{rank} \operatorname{Sym}^{m} \mathcal{Q}(d) & =\left(\begin{array}{c}
n-k+m-1 \\
m
\end{array}\right), \\
\mathfrak{c}_{1}\left(\operatorname{Sym}^{m} \mathcal{Q}(d)\right) & =\left(\begin{array}{c}
n-k+m-1 \\
n-k
\end{array}\right)+d\left(\begin{array}{c}
n-k+m-1 \\
m
\end{array}\right), \\
\operatorname{rank} \wedge^{m} \mathcal{Q}(d) & =\left(\begin{array}{c}
n-k \\
m
\end{array}\right), & \mathfrak{c}_{1}\left(\wedge^{m} \mathcal{Q}(d)\right)=\left(\begin{array}{c}
n-k-1 \\
m-1
\end{array}\right)+d\left(\begin{array}{c}
n-k \\
m
\end{array}\right) .
\end{array}
$$


Returning to the main subject of the classification, (2.17) with the rank condition (2.10) implies that the following setups are possible

$$
\begin{aligned}
\left(k, n ; \ell_{p}^{\vee}, \mathfrak{c}_{1}\left(\mathcal{F}_{p}\right)\right)=(2,4 ; 1,4) \text { with } \\
\mathcal{F}_{p}=\mathcal{O}_{V}(4), \mathcal{O}_{V}(1) \oplus \mathcal{O}_{V}(3), \mathcal{O}_{V}(2)^{\oplus 2}, \mathcal{O}_{V}(1)^{\oplus 2} \oplus \mathcal{O}_{V}(2), \mathcal{S}^{*} \oplus \mathcal{O}_{V}(3), \\
\quad \mathcal{S}^{*}(1) \oplus \mathcal{O}_{V}(1), \mathcal{O}_{V}(1)^{\oplus 4}, \mathcal{S}^{*} \oplus \mathcal{O}_{V}(1) \oplus \mathcal{O}_{V}(2), \operatorname{Sym}^{2} \mathcal{S}^{*} \oplus \mathcal{O}_{V}(1), \\
\quad \mathcal{S}^{*} \oplus \mathcal{O}_{V}(1)^{\oplus 3},\left(\mathcal{S}^{*}\right)^{\oplus 2} \oplus \mathcal{O}_{V}(2),\left(\mathcal{S}^{*}\right)^{\oplus 2} \oplus \mathcal{O}_{V}(1)^{\oplus 2},\left(\mathcal{S}^{*}\right)^{\oplus 3} \oplus \mathcal{O}_{V}(1),\left(\mathcal{S}^{*}\right)^{\oplus 4}
\end{aligned}
$$

Note that $\mathcal{S}^{*} \cong \mathcal{Q}$ on $G(2,4)$ and $\wedge^{2} \mathcal{S}^{*} \cong \mathcal{O}_{V}(1)$ when $k=2$. The example constructed from $\mathcal{F}_{p}=\mathcal{O}_{V}(4)$ with $p=1$ (i.e. $\ell=0$ ) is the complete intersection Grassmannian CalabiYau 3-fold in $G(2,4)$ with the vector bundle $\mathcal{O}_{G(2,4)}(4)$. Since $\ell_{p}^{\vee}=1$ (i.e. $p=\ell+1$ ), as discussed in the case for the quintic family (2.22), these determinantal Calabi-Yau 3-folds generically have singular points and the desingularized 3 -folds are connected through the extremal transitions (see also section 4.3.3).

Another class of solutions can be obtained by the ansatz (II) in (2.12) and the result is

$$
\begin{aligned}
\left(k, n ; \ell_{p}^{\vee}, \mathfrak{c}_{1}\left(\mathcal{F}_{p}\right)\right)=(2,28 ; 7,4) \text { with } & \\
\mathcal{F}_{p}= & \left(\mathcal{S}^{*}\right)^{\oplus 3} \oplus \mathcal{O}_{V}(1),\left(\mathcal{S}^{*}\right)^{\oplus 4}, \mathcal{Q} \oplus \mathcal{O}_{V}(3), \mathcal{Q} \oplus \mathcal{O}_{V}(1) \oplus \mathcal{O}_{V}(2), \mathcal{Q} \oplus \mathcal{O}_{V}(1)^{\oplus 3}, \\
& \mathcal{Q}^{\oplus 2} \oplus \mathcal{O}_{V}(2), \mathcal{Q}^{\oplus 2} \oplus \mathcal{O}_{V}(1)^{\oplus 2}, \mathcal{Q}^{\oplus 3} \oplus \mathcal{O}_{V}(1), \mathcal{Q}^{\oplus 4} .
\end{aligned}
$$

Here the Calabi-Yau 3-fold constructed from $\mathcal{F}_{p}=\left(\mathcal{S}^{*}\right)^{\oplus 3} \oplus \mathcal{O}_{V}(1)$ with $p=7$ (i.e. $\ell=0$ ) can be identified with the complete intersection Grassmannian Calabi-Yau 3-fold in $G(2,7)$ with the vector bundle $\mathcal{O}_{G(2,7)}(1)^{\oplus 7}$.

\subsection{3 $k \geq 3$}

In this case, we have $V=G(k, n)$. Interestingly, there exist four "infinite families" given by

$$
\begin{aligned}
\left(k, n ; \ell_{p}^{\vee}, \mathfrak{c}_{1}\left(\mathcal{F}_{p}\right)\right) & =\left(k_{i}, 5 \ell_{i} ; \ell_{i}, 5\right), \quad i \in \mathbb{N} \\
\text { with } k_{1}=4, \ell_{1} & =19, k_{i+1}=\ell_{i}, \ell_{i+1}=-k_{i}+5 \ell_{i}: \mathcal{F}_{p}=\left(\mathcal{S}^{*}\right)^{\oplus 5}, \mathcal{Q}^{\oplus 5}, \\
\left(k, n ; \ell_{p}^{\vee}, \mathfrak{c}_{1}\left(\mathcal{F}_{p}\right)\right) & =\left(k_{i}, 4 \ell_{i} ; \ell_{i}, 4\right), \quad i \in \mathbb{N} \\
\text { with } k_{1}=7, \ell_{1} & =26, k_{i+1}=\ell_{i}, \ell_{i+1}=-k_{i}+4 \ell_{i}: \mathcal{F}_{p}=\left(\mathcal{S}^{*}\right)^{\oplus 4}, \mathcal{Q}^{\oplus 4} .
\end{aligned}
$$

By using mathematical induction, one can check that the duality condition (2.9), the rank condition (2.10), and in particular $\ell_{p}^{\vee}<\operatorname{rank} \mathcal{F}_{p}$, are maintained. Since we do not impose the irreducibility condition in our analysis, it is still possible that the above infinite families can be reduced to other trivial or non-trivial examples. In any case, it is required to thoroughly investigate various topological invariants of these higher rank examples, and this would require a considerable effort and we leave this issue as an open problem.

\section{$3 \quad I$-functions and Gromov-Witten invariants}

In this section, we briefly overview the computation of genus-0 Gromov-Witten invariants using the Givental $I$-functions [21-23] (see also [26]). We will also provide a handy formula for the computations of the Gromov-Witten invariants of Grassmannian Calabi-Yau varieties, which is also applicable to the determinantal varieties. 


\subsection{Building blocks of $I$-functions}

When a Fano or a Calabi-Yau variety $X$ has a GLSM realization with gauge group $G$, one can easily construct the Givental $I$-function of $X$ by using the supersymmetric localization formula (see $[14-17,27]$ ). Here we clarify the building blocks of the $I$-function of $X$ associated with such a GLSM on the $\Omega$-deformed 2-sphere $S_{\hbar}^{2}$ which has a vector multiplet and chiral matter multiplets with $R$-charge 0 or 2 under $\mathrm{U}(1)_{R}$. The deformation parameter $\hbar$ is identified with an equivariant parameter.

Let $\mathbf{x}=\left(x_{1}, \ldots, x_{\mathrm{rk}(\mathfrak{g})}\right) \in \mathfrak{h} \otimes_{\mathbb{R}} \mathbb{C}$ be Coulomb branch parameters and $\mathbf{q}=$ $\left(q_{1}, \ldots, q_{\mathrm{rk}(\mathfrak{g})}\right) \in \mathbb{Z}^{\mathrm{rk}(\mathfrak{g})} \subset i \mathfrak{h}$ be magnetic charges for Cartan subalgebra $\mathfrak{h}$ of a Lie algebra $\mathfrak{g}$ associated with $G$, where $\operatorname{rk}(\mathfrak{g})$ denotes the rank of $\mathfrak{g}$. Here the parameters x are identified with the Chern roots of $X$ which give the total Chern class of $X$ as

$$
c(X)=\prod_{i=1}^{\mathrm{rk}(\mathfrak{g})}\left(1+x_{i}\right) .
$$

To construct the $I$-function of $X$, first we need a "classical block" associated with the subgroup $\mathrm{U}(1)^{\mathrm{c}} \subset G$, where $\mathrm{c}$ is the number of the central. The Fayet-Iliopoulos (FI) parameters $\xi_{a}$ and theta angles $\theta_{a}, a=1, \ldots, \mathrm{c}$, are associated with each $\mathrm{U}(1)^{\mathrm{c}}$ factor, and the classical block of the $I$-function is given by

$$
I_{\mathbf{q}}^{\mathrm{c}}(\mathbf{z} ; \mathbf{x} ; \hbar)=\mathrm{e}^{2 \pi \sqrt{-1} \boldsymbol{\tau}(\mathbf{x} / \hbar+\mathbf{q})}, \quad \boldsymbol{\tau}=\left\{\tau_{a}\right\}:=\sqrt{-1} \xi_{a}+\frac{1}{2 \pi} \theta_{a} .
$$

Here the parameters $\mathbf{z}=\left\{z_{a}\right\}=\mathrm{e}^{2 \pi \sqrt{-1}} \tau_{a}$ represent the exponentiated Kähler moduli of $X$, and the canonical pairing $\boldsymbol{\tau}(*)$ is defined by embedding $\boldsymbol{\tau}$ into $\mathfrak{h}^{*} \otimes_{\mathbb{R}} \mathbb{C}$.

Other contributions come from the 1-loop determinants of multiplets of the GLSM. The vector multiplet provides a block given by

$$
I_{\mathbf{q}}^{\mathrm{vec}}(\mathbf{x} ; \hbar)=\prod_{\alpha \in \Delta_{+}}(-1)^{\alpha(\mathbf{q})} \frac{\alpha(\mathbf{x})+\alpha(\mathbf{q}) \hbar}{\alpha(\mathbf{x})},
$$

where $\Delta_{+}$is the set of positive roots of $\mathfrak{g}$. In general, the GLSM also has chiral matter multiplets $\Phi$ with $R$-charge 0 and $P$ with $R$-charge 2 in a certain representation $\mathbf{R}$. Note that one can turn on a twisted mass parameter $\lambda$ while preserving supersymmetry, which is identified with an equivariant parameter. Their contributions are given as follows:

$$
I_{\mathbf{q}}^{\Phi}(\mathbf{x}, \lambda ; \hbar)= \begin{cases}\prod_{\rho \in \mathbf{R}} \prod_{p=1}^{\rho(\mathbf{q})}(\rho(\mathbf{x})+\lambda+p \hbar)^{-1}, & \text { for } \rho(\mathbf{q}) \geq 1, \\ 1, & \text { for } \rho(\mathbf{q})=0, \\ \prod_{\rho \in \mathbf{R}} \prod_{p=0}^{-\rho(\mathbf{q})-1}(\rho(\mathbf{x})+\lambda-p \hbar), & \text { for } \rho(\mathbf{q}) \leq-1,\end{cases}
$$

and

$$
I_{\mathbf{q}}^{P}(\mathbf{x}, \lambda ; \hbar)= \begin{cases}\prod_{\rho \in \mathbf{R}} \prod_{p=1}^{-\rho(\mathbf{q})}(-\rho(\mathbf{x})-\lambda+p \hbar), & \text { for } \rho(\mathbf{q}) \leq-1, \\ 1, & \text { for } \rho(\mathbf{q})=0, \\ \prod_{\rho \in \mathbf{R}} \prod_{p=0}^{\rho(\mathbf{q})-1}(-\rho(\mathbf{x})-\lambda-p \hbar)^{-1}, & \text { for } \rho(\mathbf{q}) \geq 1,\end{cases}
$$




\begin{tabular}{|c|c|c|c|}
\hline Field & $\mathrm{U}(1)$ & twisted mass & $\mathrm{U}(1)_{R}$ \\
\hline$\Phi_{i}$ & +1 & $-w_{i}$ & 0 \\
$P_{a}$ & $-d_{a}$ & $\lambda_{a}$ & 2 \\
\hline
\end{tabular}

Table 1. Matter content of the U(1) GLSM for the complete intersection variety $X_{1}$ in $\mathbb{P}^{n-1}$. Here $i=0, \ldots, n-1$ and $a=1, \ldots, r$.

where $\rho$ denotes the weight of $\mathbf{R}$. Note that the products $\alpha(*)$ and $\rho(*)$ are defined by the canonical pairing.

Combining all the above building blocks (3.2), (3.3), (3.4), and (3.5), after taking a sum over the magnetic charges q, one can construct the Givental $I$-function as (3.7), (3.19), and (4.3). As we will see next, the genus-0 Gromov-Witten invariants can be extracted from this function.

\subsection{Examples}

Here we will demonstrate how to compute genus-0 Gromov-Witten invariants via the $I$ functions for well-studied examples, and find out a useful formula for treating Grassmannian Calabi-Yau varieties.

\subsubsection{Complete intersections in $\mathbb{P}^{n-1}$}

Let us consider a complete intersection variety $X_{1}=X_{d_{1}, \ldots, d_{r}} \subset \mathbb{P}^{n-1}$ defined by the zero locus of a holomorphic section of a vector bundle $\mathcal{E}=\oplus_{a=1}^{r} \mathcal{O}_{V}\left(d_{a}\right)$ on $V=\mathbb{P}^{n-1}$ satisfying Fano or Calabi-Yau condition $\sum_{a=1}^{r} d_{a} \leq n$. Note that $\operatorname{rank} \mathcal{E}=r$ and $\mathfrak{c}_{1}(\mathcal{E})=\sum_{a=1}^{r} d_{a}$. This variety has a complex dimension

$$
\operatorname{dim} X_{1}=n-r-1
$$

and is described by a U(1) GLSM whose matter content is shown in table 1. This model has a superpotential $W=\sum_{a=1}^{r} P_{a} G_{a}(\Phi)$ where $G_{a}(\Phi)$ are homogeneous degree $d_{a}$ polynomials of the chiral matter multiplets $\Phi_{i}$.

For each matter multiplet we assign twisted masses and $\mathrm{U}(1) R$-charges as described in table 1. Combining the building blocks (3.2), (3.4) and (3.5) with the assignment in table 1 , the $I$-function in the geometric large volume phase with FI parameter $\xi>0$ is constructed as [21-23]

$$
\begin{aligned}
I_{X_{1}}^{\left\{w_{i}\right\},\left\{\lambda_{a}\right\}}(z ; x ; \hbar) & =\sum_{q=0}^{\infty} I_{q}^{\mathrm{c}}(z ; x ; \hbar)\left(\prod_{i=0}^{n-1} I_{q}^{\Phi_{i}}\left(x, w_{i} ; \hbar\right)\right)\left(\prod_{a=1}^{r} I_{q}^{P_{a}}\left(x, \lambda_{a} ; \hbar\right)\right) \\
& =z^{x / \hbar} \sum_{q=0}^{\infty} \frac{\prod_{a=1}^{r} \prod_{p=1}^{d_{a} q}\left(d_{a} x-\lambda_{a}+p \hbar\right)}{\prod_{i=0}^{n-1} \prod_{p=1}^{q}\left(x-w_{i}+p \hbar\right)} z^{q} .
\end{aligned}
$$

Geometrically $z=\mathrm{e}^{-2 \pi \xi+\sqrt{-1} \theta}$ provides the Kähler moduli parameter of $X_{1}$, and $x$ is identified with the equivariant second cohomology element of $X_{1}$ satisfying $\prod_{i=0}^{n-1}\left(x-w_{i}\right)=$ 
0 , where the twisted masses $w_{i}$ give the equivariant parameters acting on $\mathbb{P}^{n-1}$. The twisted masses $\lambda_{a}$ correspond to the equivariant parameters acting on $\mathcal{E}=\oplus_{a=1}^{r} \mathcal{O}_{V}\left(d_{a}\right)$.

Then it can be shown that the $I$-function (3.7) obeys the ordinary differential equation

$$
\left[\prod_{i=0}^{n-1}\left(\hbar \Theta_{z}-w_{i}\right)-z \prod_{a=1}^{r} \prod_{p=1}^{d_{a}}\left(\hbar d_{a} \Theta_{z}-\lambda_{a}+p \hbar\right)\right] I_{X_{1}}^{\left\{w_{i}\right\},\left\{\lambda_{a}\right\}}(z ; x ; \hbar)=0, \quad \Theta_{z}:=z \frac{d}{d z} .
$$

In the Calabi-Yau case $\sum_{a=1}^{r} d_{a}=n$ with vanishing equivariant parameters $w_{i}=\lambda_{a}=$ 0 , the differential equation (3.8) yields the Picard-Fuchs equation for the periods of the holomorphic $(n-r-1)$-form on $X_{1}$ given by [28-31]

$$
\Theta_{z}^{r}\left[\Theta_{z}^{n-r}-\left(\prod_{a=1}^{r} d_{a}\right) z \prod_{a=1}^{r} \prod_{p=1}^{d_{a}-1}\left(d_{a} \Theta_{z}+p\right)\right] I_{X_{1}}(z ; x ; \hbar)=0,
$$

where $I_{X_{1}}(z ; x ; \hbar):=I_{X_{1}}^{\{\mathbf{0}\},\{\mathbf{0}\}}(z ; x ; \hbar)$. If we expand the $I$-function around $\hbar=\infty$ as

$$
I_{X_{1}}(z ; x ; \hbar)=\sum_{k=0}^{n-r-1} I_{k}(z)\left(\frac{x}{\hbar}\right)^{k}
$$

the coefficients $I_{k}(z)$ precisely give the solutions to the Picard-Fuchs equation. One can also obtain the flat coordinate $q$ on the Kähler moduli space of $X_{1}$ through the relation

$$
\log q=\frac{I_{1}(z)}{I_{0}(z)}=\log z+O(z)
$$

called the mirror map. It has been shown in [32] that the genus-0 3-point A-model correlators $\left\langle\mathcal{O}_{h} \mathcal{O}_{h^{k}} \mathcal{O}_{h^{n-r-k-2}}\right\rangle_{\mathbb{P}^{1}}, k=1, \ldots, n-r-1$, which enumerate the number of rational curves are given by

$$
\begin{aligned}
\left\langle\mathcal{O}_{h} \mathcal{O}_{h^{k}} \mathcal{O}_{h^{n-r-k-2}}\right\rangle_{\mathbb{P}^{1}} & =\kappa \frac{\widehat{I}_{k+1}(z(q))}{\widehat{I}_{1}(z(q))} \\
& =\kappa+\sum_{d=1}^{\infty} n_{d}\left(h^{1}, h^{k}, h^{n-r-k-2}\right) \frac{q^{d}}{1-q^{d}},
\end{aligned}
$$

where $\widehat{I}_{k}(z)$ are inductively constructed from the $I$-functions as

$$
\begin{aligned}
& \widehat{I}_{0}(z)=I_{0}(z) \\
& \widehat{I}_{k}(z)=\Theta_{z} \frac{1}{\widehat{I}_{k-1}(z)} \Theta_{z} \frac{1}{\widehat{I}_{k-2}(z)} \cdots \Theta_{z} \frac{1}{\widehat{I}_{1}(z)} \Theta_{z} \frac{I_{k}(z)}{\widehat{I}_{0}(z)}, \quad k=1, \ldots, n-r-1 .
\end{aligned}
$$

Here the observable $\mathcal{O}_{h^{p}}$ is associated with the hyperplane class $h \in H^{1,1}\left(X_{1}\right)$, and

$$
\kappa=\int_{X_{1}} h^{n-r-1}=\left(\prod_{a=1}^{r} d_{a}\right) \int_{\mathbb{P}^{n-1}} h^{n-1}=\prod_{a=1}^{r} d_{a}
$$




\begin{tabular}{|c|c|c|c|}
\hline Field & $\mathrm{U}(\mathrm{k})$ & twisted mass & $\mathrm{U}(1)_{R}$ \\
\hline$\Phi_{I}$ & $\mathbf{k}$ & $-w_{I}$ & 0 \\
$P_{a}$ & $\operatorname{det}^{-d_{a}}$ & $\lambda_{a}$ & 2 \\
\hline
\end{tabular}

Table 2. Matter content of the $\mathrm{U}(k)$ GLSM for the complete intersection variety $X_{2}$ in $G(k, n)$. Here $I=1, \ldots, n$ and $a=1, \ldots, r$.

is the classical intersection number of $X_{1} . \widehat{I}_{k}(z)$ have relations

$$
\widehat{I}_{k}(z)=\widehat{I}_{n-r-k}(z), \quad k=1, \ldots, n-r-1 .
$$

Note that there is a selection rule $\sum_{i=1}^{n} p_{i}=\operatorname{dim} X_{1}+n-3$ to realize non-trivial genus$0 n$-point correlators $\left\langle\mathcal{O}_{h^{p_{1}}} \cdots \mathcal{O}_{h^{p_{n}}}\right\rangle_{\mathbb{P}^{1}}$ arising from the index theorem. The number $n_{d}\left(h^{1}, h^{k}, h^{n-r-k-2}\right)$ in (3.12) is an integer and enumerates the number of degree $d$ holomorphic maps intersecting with the cycles dual to $h, h^{k}$, and $h^{n-r-k-2}[30,31,33]$ (see also $[34,35])$.

In a special case with $k=1$, the relation $\Theta_{z}=\widehat{I}_{1}(z) \Theta_{q}$ and the so-called divisor equation $\left\langle\mathcal{O}_{h} \cdots\right\rangle_{\mathbb{P}^{1}}=\Theta_{q}\langle\cdots\rangle_{\mathbb{P}^{1}}$ imply that

$$
\left\langle\mathcal{O}_{h^{n-r-3}}\right\rangle_{\mathbb{P}^{1}}=\kappa \frac{I_{2}(z(q))}{I_{0}(z(q))}=\frac{\kappa}{2}(\log q)^{2}+\sum_{d=1}^{\infty} n_{d}\left(h^{n-r-3}\right) \operatorname{Li}_{2}\left(q^{d}\right),
$$

where $\operatorname{Li}_{p}(z)=\sum_{k=1}^{\infty} \frac{z^{k}}{k^{p}}$. Here $n_{d}\left(h^{n-r-3}\right)=n_{d}\left(h, h, h^{n-r-3}\right) / d^{2}$ is an integer which enumerates the number of degree $d$ holomorphic maps intersecting with the cycle dual to $h^{n-r-3}$. When $n-r=4$ (i.e. $\operatorname{dim} X_{1}=3$ ), by the divisor equation, (3.16) yields [36, 37]

$$
\langle *\rangle_{\mathbb{P}^{1}}=\kappa \int^{q} \frac{I_{2}\left(z\left(q^{\prime}\right)\right)}{I_{0}\left(z\left(q^{\prime}\right)\right)} \frac{d q^{\prime}}{q^{\prime}}=\frac{\kappa}{3 !}(\log q)^{3}+\sum_{d=1}^{\infty} n_{d} \operatorname{Li}_{3}\left(q^{d}\right),
$$

where the number $n_{d}=n_{d}(h) / d$ is a genus-0 integer invariant.

\subsubsection{Complete intersections in $G(k, n)$}

Let us consider a complete intersection variety $X_{2}$ defined by the zero locus of a holomorphic section of $\mathcal{E}=\oplus_{a=1}^{r} \mathcal{O}_{V}\left(d_{a}\right)$ on Grassmannian $V=G(k, n)$ satisfying Fano or Calabi-Yau condition $\sum_{a=1}^{r} d_{a} \leq n$. Note that $\operatorname{rank} \mathcal{E}=r$ and $\mathfrak{c}_{1}(\mathcal{E})=\sum_{a=1}^{r} d_{a}$. The variety $X_{2}$ has a complex dimension

$$
\operatorname{dim} X_{2}=k(n-k)-r
$$

and can be described by a $\mathrm{U}(k)$ GLSM whose matter content is given in table 2. This model has a superpotential $W=\sum_{a=1}^{r} P_{a} G_{a}(B)$ where $G_{a}(B)$ are homogeneous degree $d_{a}$ polynomials of the baryonic variables $B_{I_{1} \ldots I_{k}}=\epsilon_{i_{1} \ldots i_{k}} \Phi_{I_{1}}^{i_{1}} \ldots \Phi_{I_{k}}^{i_{k}}$ called the Plücker coordinates [38].

For each matter multiplet we assign twisted masses and $\mathrm{U}(1) R$-charges as described in table 2. Combining the associated building blocks (3.2), (3.3), (3.4) and (3.5) for the 
$\mathrm{U}(k)$ vector multiplet and the chiral multiples in table 2 , we can construct the $I$-function for $X_{2}$ in the geometric phase with large FI parameter $\xi>0$ as [39]

$$
\begin{aligned}
I_{X_{2}}^{\left\{w_{I}\right\},\left\{\lambda_{a}\right\}}(z ; \mathbf{x} ; \hbar)= & \sum_{\mathbf{q} \in\left(\mathbb{Z}_{\geq 0}\right)^{k}} I_{\mathbf{q}}^{\mathrm{c}}(z ; \mathbf{x} ; \hbar) I_{\mathbf{q}}^{\mathrm{vec}}(\mathbf{x} ; \hbar)\left(\prod_{I=1}^{n} I_{\mathbf{q}}^{\Phi_{I}}\left(\mathbf{x}, w_{I} ; \hbar\right)\right)\left(\prod_{a=1}^{r} I_{\mathbf{q}}^{P_{a}}\left(\mathbf{x}, \lambda_{a} ; \hbar\right)\right) \\
= & z^{\sum_{i=1}^{k} x_{i} / \hbar} \sum_{\mathbf{q} \in\left(\mathbb{Z}_{\geq 0}\right)^{k}}\left((-1)^{k-1} z\right)^{\sum_{i=1}^{k} q_{i}} \prod_{1 \leq i<j \leq k} \frac{x_{i}-x_{j}+\left(q_{i}-q_{j}\right) \hbar}{x_{i}-x_{j}} \\
& \times \frac{\prod_{a=1}^{r} \prod_{p=1}^{d_{a} \sum_{i=1}^{k} q_{i}}\left(d_{a} \sum_{i=1}^{k} x_{i}-\lambda_{a}+p \hbar\right)}{\prod_{I=1}^{n} \prod_{i=1}^{k} \prod_{p=1}^{q_{i}}\left(x_{i}-w_{I}+p \hbar\right)} .
\end{aligned}
$$

Geometrically $z=\mathrm{e}^{-2 \pi \xi+\sqrt{-1} \theta}$ provides the Kähler moduli parameter of $X_{2}$, and $x_{i}$ are identified with the degree 2 elements in the equivariant cohomology of $X_{2}$. The twisted masses $w_{I}$ and $\lambda_{a}$ correspond to the equivariant parameters acting on $G(k, n)$ and $\mathcal{E}=$ $\oplus_{a=1}^{r} \mathcal{O}_{V}\left(d_{a}\right)$, respectively.

Remark 3.1. For $w_{I}=0$, the cohomology ring of $G(k, n)$ is given by [40] (see [41] for the equivariant quantum cohomology ring):

$$
H^{*}(G(k, n)) \cong \mathbb{C}\left[x_{1}, \ldots, x_{k}\right]^{S_{k}} /\left(h_{n-k+1}(\mathbf{x}), \ldots, h_{n}(\mathbf{x})\right),
$$

where $S_{k}$ is the symmetric group on $k$ elements and

$$
h_{p}(\mathbf{x})=\sum_{1 \leq i_{1} \leq i_{2} \leq \ldots \leq i_{p} \leq k} x_{i_{1}} x_{i_{2}} \cdots x_{i_{p}}
$$

are the complete symmetric polynomials.

For the Calabi-Yau case $\sum_{a=1}^{r} d_{a}=n$ with vanishing equivariant parameters $w_{I}=$ $\lambda_{a}=0$, the $I$-function $I_{X_{2}}(z ; \mathbf{x} ; \hbar)=I_{X_{2}}^{\{\mathbf{0}\},\{\mathbf{0}\}}(z ; \mathbf{x} ; \hbar)$ can be expanded around $\hbar=\infty$ as

$$
I_{X_{2}}(z ; \mathbf{x} ; \hbar)=\sum_{|P|=0}^{\infty} I_{P}(z) \frac{s_{P}(\mathbf{x})}{\hbar^{|P|}}, \quad|P|=\sum_{i=1}^{k} p_{i}
$$

where $s_{P}(\mathbf{x})=s_{P}\left(x_{1}, \ldots, x_{k}\right)$ is the Schur polynomial for a partition $P=\left\{p_{1}, \ldots, p_{k}\right\}$, and note that $s_{p, 0, \ldots, 0}(\mathbf{x})=h_{p}(\mathbf{x})$ and $s_{1,1, \ldots, 1}(\mathbf{x})=e_{p}(\mathbf{x})=\sum_{1 \leq i_{1}<i_{2}<\ldots<i_{p} \leq k} x_{i_{1}} x_{i_{2}} \cdots x_{i_{p}}$. The flat coordinate which provides the mirror map is given by

$$
\log q=\frac{I_{1}(z)}{I_{0}(z)}=\log z+O(z)
$$

As a non-abelian generalization of the formula (3.16), here we conjecture that the genus-0 1-point A-model correlator

$$
\left\langle\mathcal{O}_{H}\right\rangle_{\mathbb{P}^{1}}=\frac{\kappa_{H}}{2}(\log q)^{2}+\sum_{d=1}^{\infty} n_{d}(H) \operatorname{Li}_{2}\left(q^{d}\right),
$$


for the Grassmannian Calabi-Yau variety $X_{2}$ is given by

$$
\left\langle\mathcal{O}_{H}\right\rangle_{\mathbb{P}^{1}}=\int_{X_{2}} H\left(\frac{I_{2}(z(q))}{I_{0}(z(q))} \sigma_{2}+\frac{I_{1,1}(z(q))}{I_{0}(z(q))} \sigma_{1,1}\right) .
$$

Here

$$
\kappa_{H}=\int_{X_{2}} H\left(\sigma_{2}+\sigma_{1,1}\right)=\int_{X_{2}} H \sigma_{1}^{2}
$$

is the classical intersection number associated with the Poincaré dual $H$ of a codimension $\operatorname{dim} X_{2}-2$ cycle in $X_{2}$, where $\sigma_{P}$ denotes the Poincaré dual of a Schubert cycle of codimension $|P|$ in $G(k, n)$ [42]. The numbers $n_{d}(H)$ are integer invariants associated with $H$ which are related to Gromov-Witten invariants of $X_{2}$ [11, 43-45].

The Giambelli's formula and the definition of Schur polynomials yield

$$
\sigma_{1,1}=\sigma_{1}^{2}-\sigma_{2}, \quad s_{1,1}(\mathbf{x})=\sum_{1 \leq i<j \leq k} x_{i} x_{j}=s_{1}(\mathbf{x})^{2}-s_{2}(\mathbf{x}) .
$$

Then one can reformulate the expression in (3.24) in terms of the classes $\sigma_{1}$ and $\sigma_{2}$ as

$$
\begin{aligned}
\frac{I_{2}(z)}{I_{0}(z)} \sigma_{2}+\frac{I_{1,1}(z)}{I_{0}(z)} \sigma_{1,1} & =\frac{I_{1,1}(z)}{I_{0}(z)} \sigma_{1}^{2}+\frac{I_{2}(z)-I_{1,1}(z)}{I_{0}(z)} \sigma_{2} \\
& =\frac{-I_{X_{2}}\left[x_{1}^{2}\right]+I_{X_{2}}\left[x_{1} x_{2}\right]}{I_{X_{2}}[1]} \sigma_{1}^{2}+\frac{2 I_{X_{2}}\left[x_{1}^{2}\right]-I_{X_{2}}\left[x_{1} x_{2}\right]}{I_{X_{2}}[1]} \sigma_{2},
\end{aligned}
$$

where $I_{X_{2}}[t]$ denotes the coefficient of $t$ at $\hbar=1$ in the expansion (3.21). Then, it is obvious that the first term in (3.23) is determined from the classical block. One can also see that for $k=1$ the relations $I_{X_{2}}\left[x_{1} x_{2}\right]=0$ and $\sigma_{2}=\sigma_{1}^{2}$ imply that the formula (3.24) reduces to $(3.16)$.

Now we claim that the conjectural formula (3.24) is also applicable not only for complete intersection Grassmannian Calabi-Yau varieties but also for the determinantal CalabiYau varieties, as we will see in the next section.

Remark 3.2. Instead of Pieri's formula for Schubert cycles, the intersection numbers of Grassmannian $G(k, n)$ can also be computed by Martin's formula [40]:

$\int_{G(k, n)} \prod_{|R|=k(n-k)} \sigma_{R}=\frac{(-1)^{\frac{1}{2} k(k-1)}}{k !}\left(\prod_{i=1}^{k} \oint_{x_{i}=0} \frac{d x_{i}}{2 \pi \sqrt{-1}}\right) \frac{\prod_{1 \leq i<j \leq k}\left(x_{i}-x_{j}\right)^{2}}{\prod_{i=1}^{k} x_{i}^{n}} \prod_{|R|=k(n-k)} s_{R}(\mathbf{x})$.

Utilizing this formula, one can then compute the intersection numbers of complete intersection Grassmannian Calabi-Yau varieties by considering the top Chern class of their normal bundles in $G(k, n)$. For example, (3.25) for $X_{2}$ is computed as

$$
\kappa_{H}=\int_{X_{2}} H \sigma_{1}^{2}=\int_{G(k, n)} H \sigma_{1}^{2} \prod_{a=1}^{r} d_{a} \sigma_{1} .
$$

Generic case can also be treated with a slight modification. Suppose that a Grassmannian Calabi-Yau variety $X$, defined by the zero locus of a holomorphic section of a vector bundle 
on $G(k, n)$, has a GLSM realization with a massless matter multiplet $P$ in a representation $\overline{\mathbf{R}}$ of $\mathrm{U}(k)$ with $R$-charge 2 . Then one obtains

$$
\int_{X} \prod_{|R|=\operatorname{dim} X} \sigma_{R}=\frac{(-1)^{\frac{1}{2} k(k-1)}}{k !}\left(\prod_{i=1}^{k} \oint_{x_{i}=0} \frac{d x_{i}}{2 \pi \sqrt{-1}}\right) \frac{\prod_{1 \leq i<j \leq k}\left(x_{i}-x_{j}\right)^{2}}{\prod_{i=1}^{k} x_{i}^{n}} I^{P}(\mathbf{x}) \prod_{|R|=\operatorname{dim} X} s_{R}(\mathbf{x}),
$$

where

$$
I^{P}(\mathbf{x})=\prod_{\rho \in \mathbf{R}} \rho(\mathbf{x})
$$

Let us consider the dual of the universal subbundle $\mathcal{E}=\mathcal{S}^{*}$ on $G(k, n)$. A Grassmannian Calabi-Yau variety defined by the zero locus of a holomorphic section of $\mathcal{E}=\mathcal{S}^{*}$ is described by a $\mathrm{U}(k)$ GLSM with a superpotential $W=P_{i} G(\Phi)^{i}$. Here $G(\Phi)$ is a homogeneous degree 1 polynomial of $\Phi_{I}(I=1, \ldots, n)$ in $\mathbf{k}$ of the $\mathrm{U}(k)$ gauge group with $R$-charge 0 , and $P$ in $\overline{\mathbf{k}}$ with $R$-charge 2 . For the matter multiplet $P$ with twisted mass $\lambda,(3.5)$ becomes

$$
I_{\mathbf{q}}^{P}(\mathbf{x}, \lambda ; \hbar)=\prod_{i=1}^{k} \prod_{p=1}^{q_{i}}\left(x_{i}-\lambda+p \hbar\right), \quad \text { for } P \text { in } \overline{\mathbf{k}} \text { with } R \text {-charge } 2 .
$$

Similarly, for instance, for vector bundles $\mathcal{E}=\operatorname{Sym}^{m} \mathcal{S}^{*}(d)$ and $\mathcal{E}=\wedge^{m} \mathcal{S}^{*}(d)$ we get

$$
I_{\mathbf{q}}^{P}(\mathbf{x}, \lambda ; \hbar)=\prod_{1 \leq i_{1} \leq \cdots \leq i_{m} \leq k} \prod_{p=1}^{\sum_{j=1}^{m} q_{i_{j}}+d \sum_{i=1}^{k} q_{i}}\left(\sum_{j=1}^{m} x_{i_{j}}+d \sum_{i=1}^{k} x_{i}-\lambda+p \hbar\right),
$$

with $P$ in $\operatorname{Sym}^{m} \overline{\mathbf{k}} \otimes \operatorname{det}^{-d}$ and

$$
I_{\mathbf{q}}^{P}(\mathbf{x}, \lambda ; \hbar)=\prod_{1 \leq i_{1}<\cdots<i_{m} \leq k} \prod_{p=1}^{\sum_{j=1}^{m} q_{i_{j}}+d \sum_{i=1}^{k} q_{i}}\left(\sum_{j=1}^{m} x_{i_{j}}+d \sum_{i=1}^{k} x_{i}-\lambda+p \hbar\right),
$$

with $P$ in $\wedge^{m} \overline{\mathbf{k}} \otimes \operatorname{det}^{-d}$, respectively. Using these building blocks with the help of our formula (3.24), one can obtain the genus-0 Gromov-Witten invariants of Grassmannian Calabi-Yau varieties computed e.g. in [14].

To consider a Grassmannian Calabi-Yau variety associated with the universal quotient bundle $\mathcal{E}=\mathcal{Q}$ on $G(k, n)$, a little ingenuity is needed. By tensoring $\mathcal{O}_{G(k, n)}(d)$ with the short exact sequence

$$
0 \longrightarrow \mathcal{S} \longrightarrow \mathcal{O}_{G(k, n)}^{\oplus n} \longrightarrow \mathcal{Q} \longrightarrow 0
$$

as

$$
0 \longrightarrow \mathcal{S}(d) \stackrel{f}{\longrightarrow} \mathcal{O}_{G(k, n)}(d)^{\oplus n} \stackrel{g}{\longrightarrow} \mathcal{Q}(d) \longrightarrow 0
$$




\begin{tabular}{|c|c|c|c|}
\hline Field & $\mathrm{U}(k)$ & $\mathrm{U}\left(\ell_{p}^{\vee}\right)$ & $\mathrm{U}(1)_{R}$ \\
\hline$\Phi_{a}$ & $\mathbf{k}$ & $\mathbf{1}$ & 0 \\
$X_{i}$ & $\mathbf{1}$ & $\bar{\ell}_{p}^{\vee}$ & 0 \\
$P$ & $\overline{\mathbf{R}}_{p}$ & $\ell_{p}^{\vee}$ & 2 \\
\hline
\end{tabular}

\begin{tabular}{|c|c|c|c|c|}
\hline \multirow{5}{*}{$\stackrel{\text { dual }}{\longleftrightarrow}$} & Field & $\mathrm{U}(k)$ & $\mathrm{U}(\ell)$ & $\mathrm{U}(1)_{R}$ \\
\hline & $\Phi_{a}$ & $\mathrm{k}$ & 1 & 0 \\
\hline & $\tilde{X}_{i}$ & 1 & $\ell$ & 0 \\
\hline & $\tilde{Y}$ & $\mathbf{R}_{p}$ & $\bar{\ell}$ & 0 \\
\hline & $\widetilde{P}_{i}$ & $\overline{\mathbf{R}}_{p}$ & 1 & 2 \\
\hline
\end{tabular}

Table 3. The left table describes matter content of the PAX model for the desingularized determinantal Calabi-Yau variety in $G(k, n)$, where $a=1, \ldots, n, i=1, \ldots, p$, and $\mathbf{R}_{p}$ is a representation in the gauge group $\mathrm{U}(k)$ which describes the vector bundle $\mathcal{F}_{p}$. $\mathrm{U}(1)_{R}$ denotes an $R$-charge which is assigned to the matter content. The right table describes matter content of the PAXY model which is a dual GLSM of the PAX model.

one can realize a corresponding GLSM for the vector bundle $\mathcal{E}=\mathcal{Q}(d)$ from the viewpoint of a quotient $\mathcal{O}_{G(k, n)}(d)^{\oplus n} / \operatorname{im}(f)$. The resulting model consists of $n$ matter multiplets $P_{i}$ in $\operatorname{det}^{-d}, i=1, \ldots, n$ of $\mathrm{U}(k)$ with $R$-charge 2 , and a single matter multiplet $Y$ in $\overline{\mathbf{k}} \otimes \operatorname{det}^{d}$ of $\mathrm{U}(k)$ with $R$-charge 0 [46]. The associated building block of the $I$-function without twisted mass is then given by

$$
I_{\mathbf{q}}^{\left\{P_{i}\right\} / Y}(\mathbf{x} ; \hbar)=\frac{\prod_{p=1}^{d \sum_{i=1}^{k} q_{i}}\left(d \sum_{i=1}^{k} x_{i}+p \hbar\right)^{n}}{\prod_{i=1}^{k} \prod_{p=1}^{-q_{i}+d \sum_{j=1}^{k} q_{j}}\left(-x_{i}+d \sum_{j=1}^{k} x_{j}+p \hbar\right)} .
$$

\section{I-functions of determinantal Calabi-Yau varieties}

In this section, we describe how to utilize our formula (3.24) to compute genus-0 GromovWitten invariants of the determinantal Calabi-Yau varieties. Here we focus on the desingularized determinantal Calabi-Yau variety $X_{A}$ in (2.6) which can be described by a $\mathrm{U}(k) \times \mathrm{U}\left(\ell_{p}^{\vee}\right)$ GLSM with matter content in the left of table 3. This GLSM is called a PAX model and has a superpotential [18]

$$
W_{P A X}=\sum_{i, j=1}^{p} \sum_{\alpha=1}^{\ell_{p}^{\vee}} P_{\alpha i} A(\Phi)_{i j} X_{j \alpha}
$$

The PAX model has several distinct phases. Let $\xi_{1}$ and $\xi_{2}$ be the FI parameters associated with the central $\mathrm{U}(1)$ factors of $\mathrm{U}(k)$ and $\mathrm{U}\left(\ell_{p}^{\vee}\right)$, respectively. For example, a geometric phase called a " $X_{A}$ phase" with $\xi_{1}>0$ and $\xi_{2}<0$ of the PAX model in the IR describes the variety $X_{A}$ in (2.6), and another geometric phase " $X_{A^{T}}$ phase" with $k \xi_{1}+\ell_{p}^{\vee} \xi_{2}>0$ and $\xi_{2}>0$ corresponds to an incidence correspondence constructed from the transposed matrix $A(\phi)^{T}$.

Remark 4.1. Alternatively, one can consider Seiberg-like dual [38] of the PAX model as follows. The chiral matter multiplet $P$ in the fundamental representation $\boldsymbol{\ell}_{p}^{\vee}$ under the $\mathrm{U}\left(\ell_{p}^{\vee}\right)$ factor corresponds to the vector bundle $\mathcal{S}^{*}$ on $G\left(\ell_{p}^{\vee}, p\right)$. By taking the Seiberg-like duality with respect to the gauge group $\mathrm{U}\left(\ell_{p}^{\vee}\right), \mathcal{S}^{*}$ is mapped to $\mathcal{Q}$ on $G(\ell, p)$ and as 
indicated by the short exact sequence (3.32), the chiral matter multiplet $P$ is mapped to the dual chiral matter multiplets $\widetilde{Y}$ and $\widetilde{P}_{i}$ in the right of table 3 . This dualized GLSM is called a PAXY model with gauge group $\mathrm{U}(k) \times \mathrm{U}(\ell)$ and has a superpotential given by [18]

$$
W_{P A X Y}=\sum_{i, j=1}^{p} \widetilde{P}_{i j}\left(A(\Phi)_{i j}-\sum_{\beta=1}^{\ell} \widetilde{Y}_{i \beta} \widetilde{X}_{\beta j}\right) .
$$

\subsection{I-functions and A-model correlators}

Let us consider the PAX model with massless matter multiplets shown in table 3. From the building blocks (3.2), (3.3), (3.4) and (3.5), the $I$-function of this model in the $X_{A}$ phase with FI parameters $\xi_{1}>0$ and $\xi_{2}<0$ is given by

$$
\begin{aligned}
I_{X_{A}}(z, w ; \mathbf{x}, \mathbf{y} ; \hbar)= & \sum_{(\mathbf{q}, \mathbf{r}) \in\left(\mathbb{Z}_{\geq 0}\right)^{k} \times\left(\mathbb{Z}_{\geq 0}\right)^{\ell_{p}^{\vee}}} I_{\mathbf{q}}^{\mathrm{c}}(z, w ; \mathbf{x}, \mathbf{y} ; \hbar) I_{\mathbf{q}, \mathbf{r}}^{\mathrm{vec}}(\mathbf{x}, \mathbf{y} ; \hbar) \\
& \times\left(I_{\mathbf{q}}^{\Phi}(\mathbf{x} ; \hbar)\right)^{n}\left(I_{\mathbf{r}}^{X}(\mathbf{y} ; \hbar)\right)^{p} I_{\mathbf{q}, \mathbf{r}}^{P}(\mathbf{x}, \mathbf{y} ; \hbar),
\end{aligned}
$$

where

$$
\begin{aligned}
I_{\mathbf{q}}^{\mathrm{c}}(z, w ; \mathbf{x}, \mathbf{y} ; \hbar) & =z^{\sum_{i=1}^{k} x_{i} / \hbar}\left((-1)^{k-1} z\right)^{\sum_{i=1}^{k} q_{i}} w^{\sum_{i=1}^{\ell_{p}^{\vee}} y_{i} / \hbar}\left((-1)^{\ell_{p}^{\vee}-1} w\right)^{\sum_{i=1}^{\ell_{p}^{\vee}} r_{i}}, \\
I_{\mathbf{q}, \mathbf{r}}^{\mathrm{vec}}(\mathbf{x}, \mathbf{y} ; \hbar) & =\left(\prod_{1 \leq i<j \leq k} \frac{x_{i}-x_{j}+\left(q_{i}-q_{j}\right) \hbar}{x_{i}-x_{j}}\right)\left(\prod_{1 \leq i<j \leq \ell_{p}^{\vee}} \frac{y_{i}-y_{j}+\left(r_{i}-r_{j}\right) \hbar}{y_{i}-y_{j}}\right), \\
I_{\mathbf{q}}^{\Phi}(\mathbf{x} ; \hbar) & =\frac{1}{\prod_{i=1}^{k} \prod_{d=1}^{q_{i}}\left(x_{i}+d \hbar\right)}, \quad I_{\mathbf{r}}^{X}(\mathbf{y} ; \hbar)=\frac{1}{\prod_{i=1}^{\ell_{p}^{\vee}} \prod_{d=1}^{r_{i}}\left(y_{i}+d \hbar\right)}, \\
I_{\mathbf{q}, \mathbf{r}}^{P}(\mathbf{x}, \mathbf{y} ; \hbar) & =\prod_{\rho \in \mathbf{R}_{p}} \prod_{i=1}^{\ell_{p}^{\vee}} \prod_{d=1}^{\rho(\mathbf{q})+r_{i}}\left(\rho(\mathbf{x})+y_{i}+d \hbar\right) .
\end{aligned}
$$

Here $z=\mathrm{e}^{-2 \pi \xi_{1}+\sqrt{-1} \theta_{1}}$ and $w=\mathrm{e}^{2 \pi \xi_{2}-\sqrt{-1} \theta_{2}}$ are moduli parameters associated with the central $\mathrm{U}(1)^{2} \subset \mathrm{U}(k) \times \mathrm{U}\left(\ell_{p}^{\vee}\right)$, and in particular $w$ parametrizes the blowing up in (2.3). $\mathbf{x}($ resp. y) are identified with the degree 2 elements in the cohomology of $G(k, n)$ (resp. $\left.G\left(\ell_{p}^{\vee}, p\right)\right)$.

As performed in (3.21), the $I$-function (4.3) can be expanded around $\hbar=\infty$ in terms of Schur polynomials as

$$
I_{X_{A}}(z, w ; \mathbf{x}, \mathbf{y} ; \hbar)=\sum_{|Q|,|R|=0}^{\infty} I_{Q ; R}(z, w) \frac{s_{Q}(\mathbf{x}) s_{R}(\mathbf{y})}{\hbar^{|Q|+|R|}}
$$

The flat coordinates $q_{z}$ and $q_{w}$, which provide the exponentiated Kähler moduli parameters of $X_{A}$, are given by

$$
\log q_{z}=\frac{I_{1 ; 0}(z, w)}{I_{0 ; 0}(z, w)}=\log z+O(z, w), \quad \log q_{w}=\frac{I_{0 ; 1}(z, w)}{I_{0 ; 0}(z, w)}=\log w+O(z, w) .
$$


From our conjectural formula (3.24), one can deduce a formula for the genus-0 1-point A-model correlator $\left\langle\mathcal{O}_{H}\right\rangle_{\mathbb{P}^{1}}$ in the $X_{A}$ phase as

$$
\begin{aligned}
\left\langle\mathcal{O}_{H}\right\rangle_{\mathbb{P}^{1}}= & \int_{X_{A}} H\left(\frac{I_{2 ; 0}(z, w)}{I_{0 ; 0}(z, w)} \sigma_{2}+\frac{I_{1,1 ; 0}(z, w)}{I_{0 ; 0}(z, w)} \sigma_{1,1}\right. \\
& \left.+\frac{I_{1 ; 1}(z, w)}{I_{0 ; 0}(z, w)} \sigma_{1} \tau_{1}+\frac{I_{0 ; 2}(z, w)}{I_{0 ; 0}(z, w)} \tau_{2}+\frac{I_{0 ; 1,1}(z, w)}{I_{0 ; 0}(z, w)} \tau_{1,1}\right) \\
= & \frac{\kappa_{H}^{\sigma}}{2}\left(\log q_{z}\right)^{2}+\kappa_{H}^{\sigma \tau} \log q_{z} \log q_{w}+\frac{\kappa_{H}^{\tau}}{2}\left(\log q_{w}\right)^{2}+\sum_{d_{1}, d_{2}=1}^{\infty} n_{d_{1}, d_{2}}(H) \operatorname{Li}_{2}\left(q_{z}^{d_{1}} q_{w}^{d_{2}}\right) .
\end{aligned}
$$

Here

$$
\kappa_{H}^{\sigma}=\int_{X_{A}} H \sigma_{1}^{2}, \quad \kappa_{H}^{\sigma \tau}=\int_{X_{A}} H \sigma_{1} \tau_{1}, \quad \kappa_{H}^{\tau}=\int_{X_{A}} H \tau_{1}^{2},
$$

are the classical intersection numbers associated with the Poincaré dual $H$ of a codimension $\operatorname{dim} X_{A}-2$ cycle in $X_{A}$, where $\sigma_{P}\left(\right.$ resp. $\left.\tau_{P}\right)$ is the Poincaré dual of a Schubert cycle of codimension $|P|$ in $G(k, n)$ (resp. $G\left(\ell_{p}^{\vee}, p\right)$ ). The genus-0 invariants $n_{d_{1}, d_{2}}(H)$ associated with $H$, which are related to Gromov-Witten invariants, are conjecturally integers.

Remark 4.2. When $\operatorname{dim} X_{A}=3$, analogous to the formula (3.17), the above result (4.7) yields

$$
\begin{aligned}
\langle *\rangle_{\mathbb{P}^{1}}= & \frac{\kappa_{\sigma_{1}}^{\sigma}}{3 !}\left(\log q_{z}\right)^{3}+\frac{\kappa_{\sigma_{1}}^{\sigma \tau}}{2}\left(\log q_{z}\right)^{2} \log q_{w}+\frac{\kappa_{\tau_{1}}^{\sigma \tau}}{2} \log q_{z}\left(\log q_{w}\right)^{2}+\frac{\kappa_{\tau_{1}}^{\tau}}{3 !}\left(\log q_{w}\right)^{3} \\
& +\sum_{d_{1}, d_{2}=1}^{\infty} n_{d_{1}, d_{2}} \operatorname{Li}_{3}\left(q_{z}^{d_{1}} q_{w}^{d_{2}}\right),
\end{aligned}
$$

where $n_{d_{1}, d_{2}}=n_{d_{1}, d_{2}}\left(\sigma_{1}\right) / d_{1}\left(\right.$ for $\left.d_{1} \neq 0\right)$ and $n_{d_{1}, d_{2}}=n_{d_{1}, d_{2}}\left(\tau_{1}\right) / d_{2}\left(\right.$ for $\left.d_{2} \neq 0\right)$ each provide genus-0 integer invariants.

\subsection{An algorithm to compute genus-0 invariants}

In a similar fashion to the computation (3.26), by taking the classes $\sigma_{1}, \sigma_{2}, \tau_{1}$ and $\tau_{2}$ for the special Schubert cycles, the 1-point correlator (4.7) can be evaluated with

$$
\begin{aligned}
& \frac{I_{2 ; 0}(z, w)}{I_{0 ; 0}(z, w)} \sigma_{2}+\frac{I_{1,1 ; 0}(z, w)}{I_{0 ; 0}(z, w)} \sigma_{1,1}+\frac{I_{1 ; 1}(z, w)}{I_{0 ; 0}(z, w)} \sigma_{1} \tau_{1}+\frac{I_{0 ; 2}(z, w)}{I_{0 ; 0}(z, w)} \tau_{2}+\frac{I_{0 ; 1,1}(z, w)}{I_{0 ; 0}(z, w)} \tau_{1,1} \\
& =\frac{-I_{X_{A}}\left[x_{1}^{2}\right]+I_{X_{A}}\left[x_{1} x_{2}\right]}{I_{X_{A}}[1]} \sigma_{1}^{2}+\frac{2 I_{X_{A}}\left[x_{1}^{2}\right]-I_{X_{A}}\left[x_{1} x_{2}\right]}{I_{X_{2}}[1]} \sigma_{2}+\frac{I_{X_{A}}\left[x_{1} y_{1}\right]}{I_{X_{A}}[1]} \sigma_{1} \tau_{1} \\
& \quad+\frac{-I_{X_{A}}\left[y_{1}^{2}\right]+I_{X_{A}}\left[y_{1} y_{2}\right]}{I_{X_{A}}[1]} \tau_{1}^{2}+\frac{2 I_{X_{A}}\left[y_{1}^{2}\right]-I_{X_{A}}\left[y_{1} y_{2}\right]}{I_{X_{2}}[1]} \tau_{2},
\end{aligned}
$$

where $I_{X_{A}}[t]$ denotes the coefficient of $t$ at $\hbar=1$ in the expansion (4.5). From the coefficients $I_{X_{A}}[t]$ and the classical intersection numbers

$$
\int_{X_{A}} H \sigma_{1}^{2}, \quad \int_{X_{A}} H \sigma_{2}, \quad \int_{X_{A}} H \sigma_{1} \tau_{1}, \quad \int_{X_{A}} H \tau_{1}^{2}, \quad \int_{X_{A}} H \tau_{2}
$$


one can compute the 1-point A-model correlator (4.7) and obtain the integer invariants. The classical intersection numbers can be computed by Martin's formula (3.28) as

$$
\begin{aligned}
\int_{X_{A}|Q|+|R|=\operatorname{dim} X_{A}} \sigma_{Q} \tau_{R}= & \frac{(-1)^{\frac{1}{2} k(k-1)+\frac{1}{2} \ell_{p}^{\vee}\left(\ell_{p}^{\vee}-1\right)}}{k ! \ell_{p}^{\vee} !}\left(\prod_{i=1}^{k} \oint_{x_{i}=0} \frac{d x_{i}}{2 \pi \sqrt{-1}}\right)\left(\prod_{i=1}^{\ell_{p}^{\vee}} \oint_{y_{i}=0} \frac{d y_{i}}{2 \pi \sqrt{-1}}\right) \\
& \times \frac{\prod_{1 \leq i<j \leq k}\left(x_{i}-x_{j}\right)^{2}}{\prod_{i=1}^{k} x_{i}^{n}} \frac{\prod_{1 \leq i<j \leq \ell_{p}^{\vee}}\left(y_{i}-y_{j}\right)^{2}}{\prod_{i=1}^{\ell_{p}^{\vee}} y_{i}^{p}} I^{P}(\mathbf{x}, \mathbf{y}) \\
& \times \prod_{|Q|+|R|=\operatorname{dim} X_{A}} s_{Q}(\mathbf{x}) s_{R}(\mathbf{y}),
\end{aligned}
$$

where

$$
I^{P}(\mathbf{x}, \mathbf{y})=\prod_{\rho \in \mathbf{R}_{p}} \prod_{i=1}^{\ell_{p}^{\vee}}\left(\rho(\mathbf{x})+y_{i}\right) .
$$

\subsection{Illustrative examples of the computations}

Here we will consider several examples of the desingularized determinantal Calabi-Yau 3folds investigated in section 2.3 and compute their genus- 0 invariants $n_{d_{1}, d_{2}}$ defined in (4.9). ${ }^{6}$

\subsubsection{Quintic family}

The determinantal Calabi-Yau 3-folds in (2.22) are connected with the famous quintic Calabi-Yau 3-fold with $\left(h^{1,1}, h^{2,1}\right)=(1,101)$ which can be described as a "trivial" determinantal 3-fold with $\mathcal{F}_{p}=\mathcal{O}_{V}(5)$ on $V=\mathbb{P}^{4}$. In terms of the parameters in section 3.2.1, the quintic 3-fold is characterized as $X_{1}$ with $n=5, r=1$ and $d_{1}=5$. The classical intersection number (3.14) of $X_{1}$ is given by $\kappa=5$ and the genus- 0 invariants $n_{d}$ in (3.17) are well-known to be [36]

$$
\begin{aligned}
& n_{1}=2875, n_{2}=609250, n_{3}=317206375, n_{4}=242467530000, \\
& n_{5}=229305888887625, \ldots
\end{aligned}
$$

The quintic family can be described by GLSMs with $\mathrm{U}(1) \times \mathrm{U}(1)$ gauge group. Following section 4.2 and appendix $\mathrm{B}$, one can compute topological invariants of the quintic family as summarized in table 4 , which is consistent with the previous works. Here one can also check that $h^{1,0}=0$. By comparing (4.13) with the entries $n_{d_{1}, d_{2}}$ in table 4 of each determinantal 3 -fold, we see that they exhibit a behavior of the extremal transition [47] (see also [10]):

$$
n_{d}=\sum_{d_{2}=0}^{N} n_{d, d_{2}}
$$

where $N$ is a certain finite positive integer.

\footnotetext{
${ }^{6}$ We only focus on the determinantal varieties described by $\mathrm{U}(k) \times \mathrm{U}\left(\ell_{p}^{\vee}\right)$ PAX models with $k \leq 2, \ell_{p}^{\vee} \leq 2$. In appendix $\mathrm{C}$ we summarize our computational results for several determinantal Calabi-Yau 4-folds.
} 


\begin{tabular}{|c|c|c|c|c|c|c|c|}
\hline \multicolumn{8}{|c|}{$\mathcal{F}_{p}=\mathcal{O}_{V}(1) \oplus \mathcal{O}_{V}(4):\left(h^{1,1}, h^{2,1}\right)=(2,86)$} \\
\hline \multicolumn{3}{|c|}{ Intersection numbers } & \multicolumn{5}{|c|}{$\overline{\sigma_{1}^{3}=5,} \quad \sigma_{1}^{2} \tau_{1}=4, \quad \sigma_{1} \tau_{1}^{2}=0, \quad \tau_{1}^{3}=0$} \\
\hline$n_{d_{1}, d_{2}}$ & $d_{1}=0$ & 1 & 2 & 3 & 4 & 5 & 6 \\
\hline$d_{2}=0$ & & 640 & 10032 & 288384 & 10979984 & 495269504 & 24945542832 \\
\hline 1 & 16 & 2144 & 231888 & 23953120 & 2388434784 & 232460466048 & 22229609118768 \\
\hline 2 & 0 & 120 & 356368 & 144785584 & 36512550816 & 7251261673320 & 1242876017216016 \\
\hline 3 & 0 & -32 & 14608 & 144051072 & 115675981232 & 50833652046112 & 16156774167471792 \\
\hline 4 & 0 & 3 & -4920 & 5273880 & 85456640608 & 106397389165188 & 69178537204963920 \\
\hline 5 & 0 & 0 & 1680 & -1505472 & 3018009984 & 62800738246496 & 107220234702633360 \\
\hline 6 & 0 & 0 & -480 & 512136 & -748922304 & 2196615443648 & 52910679981204144 \\
\hline \multicolumn{8}{|c|}{$\mathcal{F}_{p}=\mathcal{O}_{V}(2) \oplus \mathcal{O}_{V}(3):\left(h^{1,1}, h^{2,1}\right)=(2,66)$} \\
\hline \multicolumn{3}{|c|}{ Intersection numbers } & \multicolumn{5}{|c|}{$\sigma_{1}^{3}=5, \quad \sigma_{1}^{2} \tau_{1}=6, \quad \sigma_{1} \tau_{1}^{2}=0, \quad \tau_{1}^{3}=0$} \\
\hline$n_{d_{1}, d_{2}}$ & $d_{1}=0$ & 1 & 2 & 3 & 4 & 5 & 6 \\
\hline$d_{2}=0$ & & 366 & 2670 & 35500 & 606264 & 12210702 & 273649804 \\
\hline 1 & 36 & 1584 & 73728 & 3286224 & 142523712 & 6060689280 & 253954899504 \\
\hline 2 & 0 & 909 & 255960 & 34736049 & 3387935304 & 273906849222 & 19594379113848 \\
\hline 3 & 0 & 16 & 231336 & 106245024 & 23702767680 & 3623779411776 & 436922554063224 \\
\hline 4 & 0 & 0 & 45216 & 119474748 & 66922830504 & 19938817169442 & 4093759996324344 \\
\hline 5 & 0 & 0 & 360 & 48046176 & 85607985132 & 53346064121712 & 19206910967576760 \\
\hline 6 & 0 & 0 & -20 & 5357838 & 49765200024 & 74247746393898 & 49456242071288532 \\
\hline \multicolumn{8}{|c|}{$\mathcal{F}_{p}=\mathcal{O}_{V}(1) \oplus \mathcal{O}_{V}(2)^{\oplus 2}:\left(h^{1,1}, h^{2,1}\right)=(2,58)$} \\
\hline \multicolumn{3}{|c|}{ Intersection numbers } & \multicolumn{5}{|c|}{$\sigma_{1}^{3}=5, \quad \sigma_{1}^{2} \tau_{1}=8, \quad \sigma_{1} \tau_{1}^{2}=4, \quad \tau_{1}^{3}=0$} \\
\hline$n_{d_{1}, d_{2}}$ & $d_{1}=0$ & 1 & 2 & 3 & 4 & 5 & 6 \\
\hline$d_{2}=0$ & & 144 & 140 & 144 & 112 & 144 & 140 \\
\hline 1 & 44 & 1120 & 13520 & 107264 & 645048 & 3190528 & 13669600 \\
\hline 2 & 0 & 1354 & 113916 & 3627224 & 68006448 & 901242596 & 9287483360 \\
\hline 3 & 0 & 256 & 258840 & 29390080 & 1463601384 & 44141205824 & 937689927488 \\
\hline 4 & 0 & 1 & 183690 & 89360780 & 11490671144 & 741564140238 & 30303625673624 \\
\hline 5 & 0 & 0 & 37896 & 115185728 & 41359928372 & 5682155162688 & 434288936956304 \\
\hline 6 & 0 & 0 & 1248 & 64102328 & 74832601592 & 22827028536708 & 3267218929443668 \\
\hline
\end{tabular}

\begin{tabular}{|c|rrrrrrr|}
\hline \hline \multicolumn{8}{|c|}{$\mathcal{F}_{p}=\mathcal{O}_{V}(1)^{\oplus 2} \oplus \mathcal{O}_{V}(3):\left(h^{1,1}, h^{2,1}\right)=(2,68)$} \\
\hline \multicolumn{7}{|c|}{ Intersection numbers } & \multicolumn{7}{c|}{$\sigma_{1}^{3}=5}$, & $\sigma_{1}^{2} \tau_{1}=7$, & $\sigma_{1} \tau_{1}^{2}=3$, & $\tau_{1}^{3}=0$ \\
\hline$n_{d_{1}, d_{2}}$ & $d_{1}=0$ & 1 & 2 & 3 & 4 & 5 & 132 \\
\hline$d_{2}=0$ & & 204 & 204 & 132 & 204 & 204 & 123786248 \\
1 & 34 & 1348 & 26843 & 338016 & 3050972 & 21359344 & 103385827082 \\
2 & 0 & 1290 & 179490 & 9621696 & 299056816 & 6401442680 & 9166825459347 \\
3 & 0 & 35 & 292557 & 59496360 & 5101530190 & 260050051116 & 235659178171360 \\
4 & 0 & -2 & 108312 & 127400436 & 29874798664 & 3367972159714 & 2557795024380895 \\
5 & 0 & 0 & 1909 & 97863426 & 75032773743 & 18958650256980 & \\
6 & 0 & 0 & -68 & 22115268 & 84738954674 & 52879556793440 & 13984934136290076 \\
\hline
\end{tabular}

\begin{tabular}{|c|rrrrrrr|}
\hline \hline \multicolumn{7}{|c|}{$\mathcal{F}_{p}=\mathcal{O}_{V}(1)^{\oplus 3} \oplus \mathcal{O}_{V}(2):\left(h^{1,1}, h^{2,1}\right)=(2,56)$} \\
\hline \multicolumn{7}{|c|}{ Intersection numbers } & \multicolumn{7}{c|}{$\sigma_{1}^{3}=5}$, & $\sigma_{1}^{2} \tau_{1}=9$, & $\sigma_{1} \tau_{1}^{2}=7$, & $\tau_{1}^{3}=2$ & \\
\hline$n_{d_{1}, d_{2}}$ & $d_{1}=0$ & 1 & 2 & 3 & 4 & 5 & 0 \\
\hline$d_{2}=0$ & & 84 & 10 & 0 & 0 & 0 & 865 \\
1 & 46 & 865 & 4461 & 9380 & 9380 & 4461 & 27724124 \\
2 & 0 & 1478 & 60360 & 760580 & 4423324 & 14207450 & 11758507011 \\
3 & 0 & 438 & 211547 & 10517154 & 200833886 & 1987023580 & 926085646998 \\
4 & 0 & 10 & 238798 & 51571964 & 2762153102 & 67275586298 & 26171128616181 \\
5 & 0 & 0 & 86203 & 107216585 & 16493768487 & 916157767777 & 353098716104028 \\
6 & 0 & 0 & 7826 & 99623760 & 48905658096 & 6224190580040 &
\end{tabular}




\begin{tabular}{|c|rr|rrrrr|}
\hline \multicolumn{7}{|c|}{$\mathcal{F}_{p}=\mathcal{O}_{V}(1)^{\oplus 5}:\left(h^{1,1}, h^{2,1}\right)=(2,52)[24,48,49]$} \\
\hline \multicolumn{2}{|c}{ Intersection numbers } & \multicolumn{7}{c|}{$\sigma_{1}^{3}=5}$, & $\sigma_{1}^{2} \tau_{1}=10$, & $\sigma_{1} \tau_{1}^{2}=10$, & $\tau_{1}^{3}=5$ & \\
\hline$n_{d_{1}, d_{2}}$ & $d_{1}=0$ & 1 & 2 & 3 & 4 & 5 & 0 \\
\hline$d_{2}=0$ & & 50 & 0 & 0 & 0 & 0 & 0 \\
1 & 50 & 650 & 1475 & 650 & 50 & 0 & 29350 \\
2 & 0 & 1475 & 29350 & 148525 & 250550 & 148525 & 110273275 \\
3 & 0 & 650 & 148525 & 3270050 & 24162125 & 75885200 & 22945154050 \\
4 & 0 & 50 & 250550 & 24162125 & 545403950 & 5048036025 & 1231494256550 \\
5 & 0 & 0 & 148525 & 75885200 & 5048036025 & 114678709000 & 27995704239850 \\
6 & 0 & 0 & 29350 & 110273275 & 22945154050 & 1231494256550 & \\
\hline
\end{tabular}

Table 4. Genus-0 invariants of determinantal 3 -folds in $(2.22)$ with $V=\mathbb{P}^{4}$.

\subsubsection{Determinantal Calabi-Yau 3-folds in (2.23)}

Next, let us consider the determinantal Calabi-Yau 3-folds described in (2.23) with $p \neq 2$. These examples can be described by GLSMs with $\mathrm{U}(1) \times \mathrm{U}(2)$ gauge group. Using the methodology we established in section 4.2 , one can obtain the genus-0 invariants summarized in table 5 . Here one can also check that $h^{1,0}=0$.

\subsubsection{Determinantal Calabi-Yau 3-folds in (2.25)}

In a similar spirit to the quintic family discussed above, the determinantal Calabi-Yau 3 -folds in (2.25) are connected with the complete intersection Calabi-Yau 3-fold with $\left(h^{1,1}, h^{2,1}\right)=(1,89)$ corresponding to the "trivial" determinantal Calabi-Yau 3-fold with $\mathcal{F}_{p}=\mathcal{O}_{V}(4)$ on $V=G(2,4)$, namely $X_{2}$ with $k=2, n=4, r=1$ and $d_{1}=4$ in the language of section 3.2.2. This family is described by GLSMs with $\mathrm{U}(2) \times \mathrm{U}(1)$ gauge group.

The classical intersection numbers (3.25) of $X_{2}$ are given by $\sigma_{1}^{3}=8, \sigma_{1} \sigma_{2}=4$, and the genus-0 invariants $n_{d}=n_{d}\left(\sigma_{1}\right) / d$ in $(3.24)$ are

$$
n_{1}=1280, n_{2}=92288, n_{3}=15655168, n_{4}=3883902528, n_{5}=1190923282176, \ldots
$$

The genus-0 invariants for other determinantal Calabi-Yau 3-folds in (2.25) are summarized in table 6 , where one can check that they exhibit the behavior (4.14) of the extremal transition and $h^{1,0}=0$.

Note that, via the incidence correspondence (2.6), a geometric phase of the determinantal Calabi-Yau variety with $\mathcal{F}_{p}=\mathcal{O}_{V}(1)^{\oplus 4}$ on $V=\mathbb{P}^{7}$ in $(2.23)$ can be identified with a geometric phase of the variety with $\mathcal{F}_{p}=\left(\mathcal{S}^{*}\right)^{\oplus 4}$ on $V=G(2,4)$ in (2.25) [18]. Indeed, by taking $d_{1} \leftrightarrow d_{2}$, the genus-0 invariants $n_{d_{1}, d_{2}}$ of the former coincide with the genus-0 invariants of the latter [10]. 


\begin{tabular}{|c|c|c|c|c|c|c|c|c|}
\hline \multicolumn{9}{|c|}{$\mathcal{F}_{p}=\mathcal{O}_{V}(1)^{\oplus 2} \oplus \mathcal{O}_{V}(2):\left(h^{1,1}, h^{2,1}\right)=(2,58)$ (Gulliksen-Negård Calabi-Yau 3-fold [18, 25]) } \\
\hline \multicolumn{3}{|c|}{ Intersection numbers } & \multicolumn{3}{|c|}{$\sigma_{1}^{3}=17, \quad \sigma_{1}^{2} \tau_{1}=10$} & \multirow{2}{*}{$\begin{array}{r}\sigma_{1} \tau_{1}^{2}=4, \quad \\
5\end{array}$} & \multirow{2}{*}{$\begin{array}{c}\tau_{1}^{3}=0 \\
6\end{array}$} & \multirow{2}{*}{$\begin{array}{r}\tau_{1} \tau_{2}=0 \\
7\end{array}$} \\
\hline$n_{d_{1}, d_{2}}$ & $d_{1}=0$ & 1 & 2 & 3 & 4 & & & \\
\hline$d_{2}=0$ & & 156 & 116 & 156 & 112 & & 116 & 156 \\
\hline 1 & 0 & 256 & 6656 & 63232 & 415232 & 2159360 & 9583104 & 37772288 \\
\hline 2 & 0 & 1 & 1248 & 193678 & 8278144 & 172114785 & 2326878112 & 23641531470 \\
\hline 3 & 0 & 0 & 0 & 10496 & 5211136 & 592671744 & 28906081792 & 822717728768 \\
\hline 4 & 0 & 0 & 0 & 0 & 111712 & 136564760 & 31768995672 & 2999009092032 \\
\hline 5 & 0 & 0 & 0 & 0 & 0 & 1394944 & 3522539520 & 1444421355520 \\
\hline 6 & 0 & 0 & 0 & 0 & 0 & 0 & 19318752 & 89779792749 \\
\hline \multicolumn{9}{|c|}{$\mathcal{F}_{p}=\mathcal{O}_{V}(1)^{\oplus 4}:\left(h^{1,1}, h^{2,1}\right)=(2,34)$ (Gulliksen-Negård Calabi-Yau 3-fold $\left.[10,25,50,51]\right)$} \\
\hline \multicolumn{3}{|c|}{ Intersection numbers } & \multicolumn{3}{|c|}{$\sigma_{1}^{3}=20, \quad \sigma_{1}^{2} \tau_{1}=20$} & $\sigma_{1} \tau_{1}^{2}=16$ & $\sigma_{1} \tau_{2}=6, \quad \tau_{1}^{3}$ & $\tau_{1} \tau_{2}=4$ \\
\hline$n_{d_{1}, d_{2}}$ & $d_{1}=0$ & 1 & 2 & 3 & 4 & 5 & 6 & 7 \\
\hline$d_{2}=0$ & & 56 & 0 & 0 & 0 & 0 & 0 & 0 \\
\hline 1 & 0 & 192 & 896 & 192 & 0 & 0 & 0 & 0 \\
\hline 2 & 0 & 56 & 2544 & 23016 & 41056 & 23016 & 2544 & 56 \\
\hline 3 & 0 & 0 & 896 & 52928 & 813568 & 3814144 & 6292096 & 3814144 \\
\hline 4 & 0 & 0 & 0 & 23016 & 1680576 & 35857016 & 284749056 & 933789504 \\
\hline 5 & 0 & 0 & 0 & 192 & 813568 & 66781440 & 1784024064 & 20090433088 \\
\hline 6 & 0 & 0 & 0 & 0 & 41056 & 35857016 & 3074369392 & 96591652016 \\
\hline
\end{tabular}

Table 5. Genus-0 invariants of determinantal 3 -folds in (2.23) with $V=\mathbb{P}^{7}$.

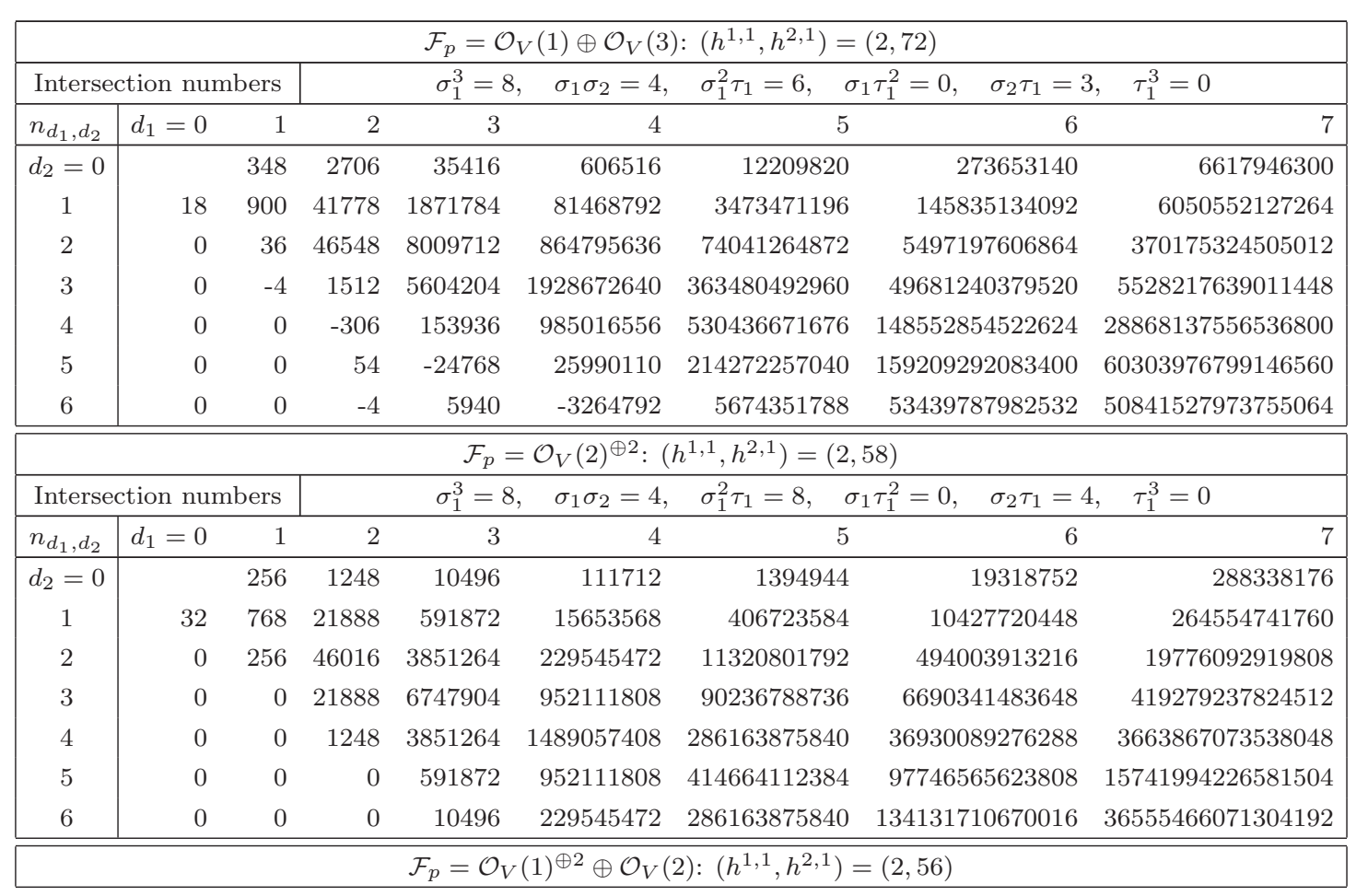




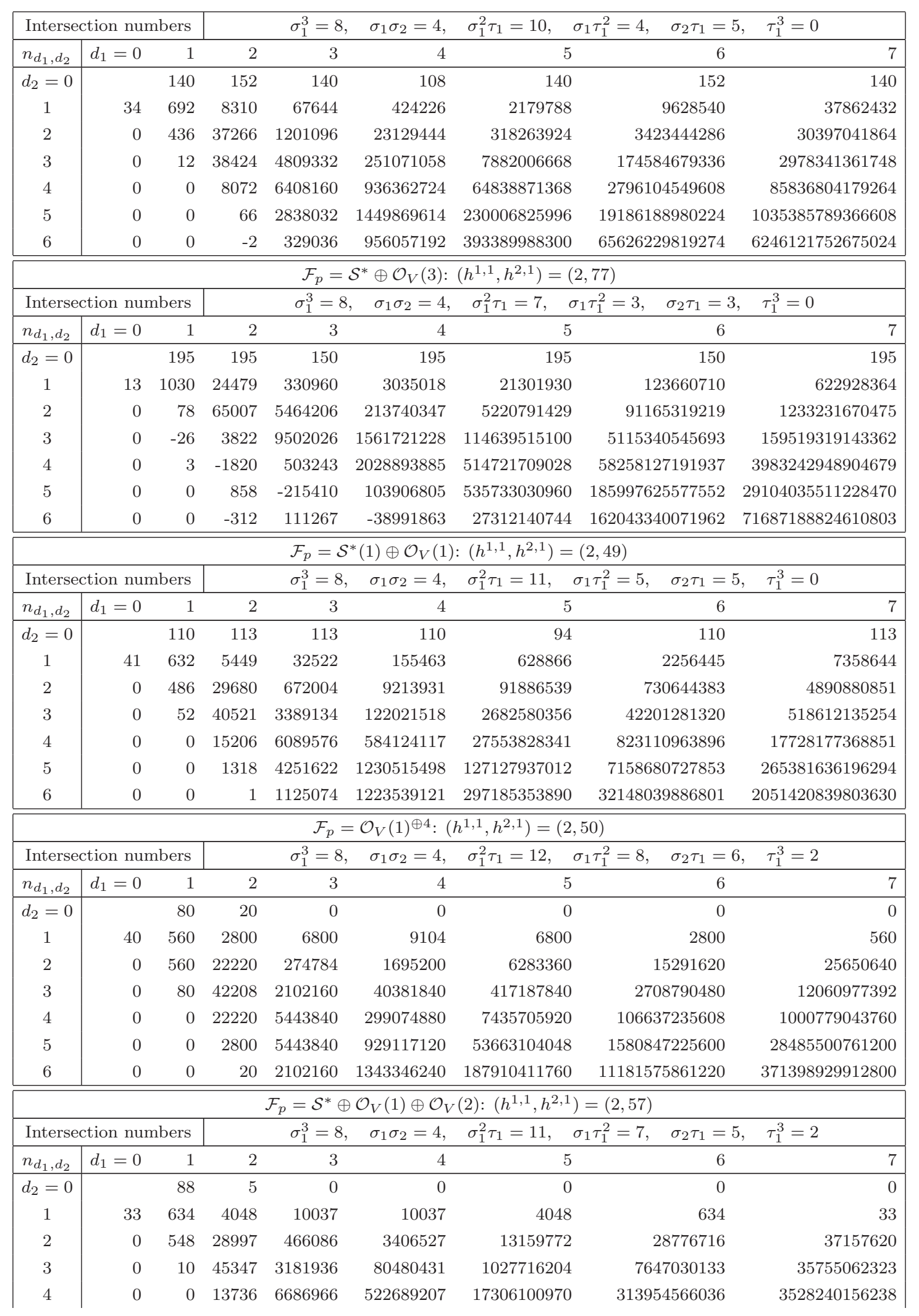




\begin{tabular}{|c|c|c|c|c|c|c|c|c|}
\hline 5 & 0 & 0 & 165 & 4524366 & 1312841562 & 108179999795 & 4280740941876 & 99081868036162 \\
\hline 6 & 0 & 0 & -10 & 780282 & 1358341003 & 305921060292 & 25988397030539 & 1167267498525808 \\
\hline \multicolumn{9}{|c|}{$\mathcal{F}_{p}=\operatorname{Sym}^{2} \mathcal{S}^{*} \oplus \mathcal{O}_{V}(1):\left(h^{1,1}, h^{2,1}\right)=(2,32)$} \\
\hline \multicolumn{3}{|c|}{ Intersection numbers } & & $\sigma_{1}^{3}=8$ & $\sigma_{1} \sigma_{2}=4$ & $\sigma_{1}^{2} \tau_{1}=14, \quad \sigma$ & \multirow{2}{*}{$\begin{array}{rr}\sigma_{1} \tau_{1}^{2}=12, & \sigma_{2} \tau_{1}= \\
5 & 6\end{array}$} & $\tau_{1}^{3}=4$ \\
\hline$n_{d_{1}, d_{2}}$ & $d_{1}=0$ & 1 & 2 & 3 & 4 & 5 & & 7 \\
\hline$d_{2}=0$ & & 20 & 22 & 0 & 0 & 0 & 0 & 0 \\
\hline 1 & 58 & 348 & 870 & 1160 & 870 & 348 & 58 & 0 \\
\hline 2 & 0 & 844 & 9460 & 42320 & 115744 & 200724 & 244280 & 200724 \\
\hline 3 & 0 & 68 & 35968 & 541140 & 3646870 & 14883488 & 41436000 & 83496920 \\
\hline 4 & 0 & 0 & 34722 & 2839040 & 47787096 & 402821800 & 2153902504 & 8105770980 \\
\hline 5 & 0 & 0 & 11050 & 5898656 & 298453714 & 5287652400 & 51848056504 & 335849637824 \\
\hline 6 & 0 & 0 & 196 & 4822716 & 908058576 & 37135584632 & 678692927028 & 7409928380632 \\
\hline \multicolumn{9}{|c|}{$\mathcal{F}_{p}=\mathcal{S}^{*} \oplus \mathcal{O}_{V}(1)^{\oplus 3}:\left(h^{1,1}, h^{2,1}\right)=(2,49)$} \\
\hline \multicolumn{3}{|c|}{ Intersection numbers } & & $\sigma_{1}^{3}=8$ & $\sigma_{1} \sigma_{2}=4$ & $\sigma_{1}^{2} \tau_{1}=13, \quad \sigma$ & \multirow{2}{*}{$\begin{array}{rr}\sigma_{1} \tau_{1}^{2}=11, & \sigma_{2} \tau_{1}= \\
5 & 6 \\
\end{array}$} & $\tau_{1}^{3}=5$ \\
\hline$n_{d_{1}, d_{2}}$ & $d_{1}=0$ & 1 & 2 & 3 & 4 & 5 & & 7 \\
\hline$d_{2}=0$ & & 52 & 1 & 0 & 0 & 0 & 0 & 0 \\
\hline 1 & 41 & 486 & 1318 & 917 & 113 & 0 & 0 & 0 \\
\hline 2 & 0 & 632 & 15206 & 94206 & 216954 & 202196 & 72260 & 7686 \\
\hline 3 & 0 & 110 & 40521 & 1125074 & 10519903 & 43910603 & 91555625 & 99039844 \\
\hline 4 & 0 & 0 & 29680 & 4251622 & 124486831 & 1484582184 & 8931510318 & 29965206018 \\
\hline 5 & 0 & 0 & 5449 & 6089576 & 579108969 & 17340098333 & 242953144372 & 1875605165389 \\
\hline 6 & 0 & 0 & 113 & 3389134 & 1223539121 & 92586552714 & 2802737114627 & 44031493485406 \\
\hline \multicolumn{9}{|c|}{$\mathcal{F}_{p}=\left(\mathcal{S}^{*}\right)^{\oplus 2} \oplus \mathcal{O}_{V}(2):\left(h^{1,1}, h^{2,1}\right)=(2,56)$} \\
\hline \multicolumn{3}{|c|}{ Intersection numbers } & & $\sigma_{1}^{3}=8$ & $\sigma_{1} \sigma_{2}=4$ & $\sigma_{1}^{2} \tau_{1}=12, \quad \sigma$ & \multirow{2}{*}{$\begin{array}{rr}\sigma_{1} \tau_{1}^{2}=10, & \sigma_{2} \tau_{1}= \\
5 & 6\end{array}$} & $\tau_{1}^{3}=5$ \\
\hline$n_{d_{1}, d_{2}}$ & $d_{1}=0$ & 1 & 2 & 3 & 4 & 5 & & 7 \\
\hline$d_{2}=0$ & & 48 & -2 & 0 & 0 & 0 & 0 & 0 \\
\hline 1 & 34 & 544 & 1719 & 544 & 34 & 0 & 0 & 0 \\
\hline 2 & 0 & 688 & 19704 & 138352 & 291762 & 138352 & 19704 & 688 \\
\hline 3 & 0 & 0 & 48165 & 1682784 & 18006204 & 75544928 & 126642213 & 75544928 \\
\hline 4 & 0 & 0 & 22206 & 5807280 & 214145556 & 2945951712 & 18597811286 & 57190487824 \\
\hline 5 & 0 & 0 & 561 & 6279840 & 910538594 & 34261029504 & 557526592367 & 4630265286624 \\
\hline 6 & 0 & 0 & -68 & 1729152 & 1545311902 & 167569246816 & 6356737689516 & 116628229665712 \\
\hline \multicolumn{9}{|c|}{$\mathcal{F}_{p}=\left(\mathcal{S}^{*}\right)^{\oplus 2} \oplus \mathcal{O}_{V}(1)^{\oplus 2}:\left(h^{1,1}, h^{2,1}\right)=(2,46)$} \\
\hline \multicolumn{3}{|c|}{ Intersection numbers } & & $\sigma_{1}^{3}=8$ & $\sigma_{1} \sigma_{2}=4$ & $\sigma_{1}^{2} \tau_{1}=14, \quad \sigma$ & \multirow{2}{*}{$\begin{array}{lr}\sigma_{1} \tau_{1}^{2}=14, \quad \sigma_{2} \tau_{1}= \\
5\end{array}$} & $\tau_{1}^{3}=9$ \\
\hline$n_{d_{1}, d_{2}}$ & $d_{1}=0$ & 1 & 2 & 3 & 4 & 5 & & 7 \\
\hline$d_{2}=0$ & & 28 & 0 & 0 & 0 & 0 & 0 & 0 \\
\hline 1 & 44 & 404 & 579 & 28 & -2 & 0 & 0 & 0 \\
\hline 2 & 0 & 708 & 9486 & 26276 & 15912 & 432 & -2 & 0 \\
\hline 3 & 0 & 140 & 35891 & 511640 & 2079058 & 2757236 & 1011037 & 29956 \\
\hline 4 & 0 & 0 & 36284 & 2887060 & 41253512 & 209384984 & 432768018 & 355277816 \\
\hline 5 & 0 & 0 & 9641 & 5964836 & 295048376 & 4172615020 & 24615473481 & 67966106564 \\
\hline 6 & 0 & 0 & 406 & 4749072 & 923105328 & 35005695588 & 489248692862 & 3213917918364 \\
\hline \multicolumn{9}{|c|}{$\mathcal{F}_{p}=\left(\mathcal{S}^{*}\right)^{\oplus 3} \oplus \mathcal{O}_{V}(1):\left(h^{1,1}, h^{2,1}\right)=(2,41)$} \\
\hline \multicolumn{3}{|c|}{ Intersection numbers } & & $\sigma_{1}^{3}=8$ & $\sigma_{1} \sigma_{2}=4$ & $\sigma_{1}^{2} \tau_{1}=15, \quad \sigma_{1}$ & ${ }_{1}^{2}=17, \quad \sigma_{2} \tau_{1}=$ & $\tau_{1}^{3}=14$ \\
\hline$n_{d_{1}, d_{2}}$ & $d_{1}=0$ & 1 & 2 & 3 & 4 & 5 & 6 & 7 \\
\hline$d_{2}=0$ & & 10 & 0 & 0 & 0 & 0 & 0 & 0 \\
\hline 1 & 49 & 308 & 231 & 0 & 0 & 0 & 0 & 0 \\
\hline 2 & 0 & 794 & 5349 & 5729 & 231 & 0 & 0 & 0 \\
\hline 3 & 0 & 168 & 29491 & 190382 & 287583 & 76182 & 78 & 0 \\
\hline
\end{tabular}




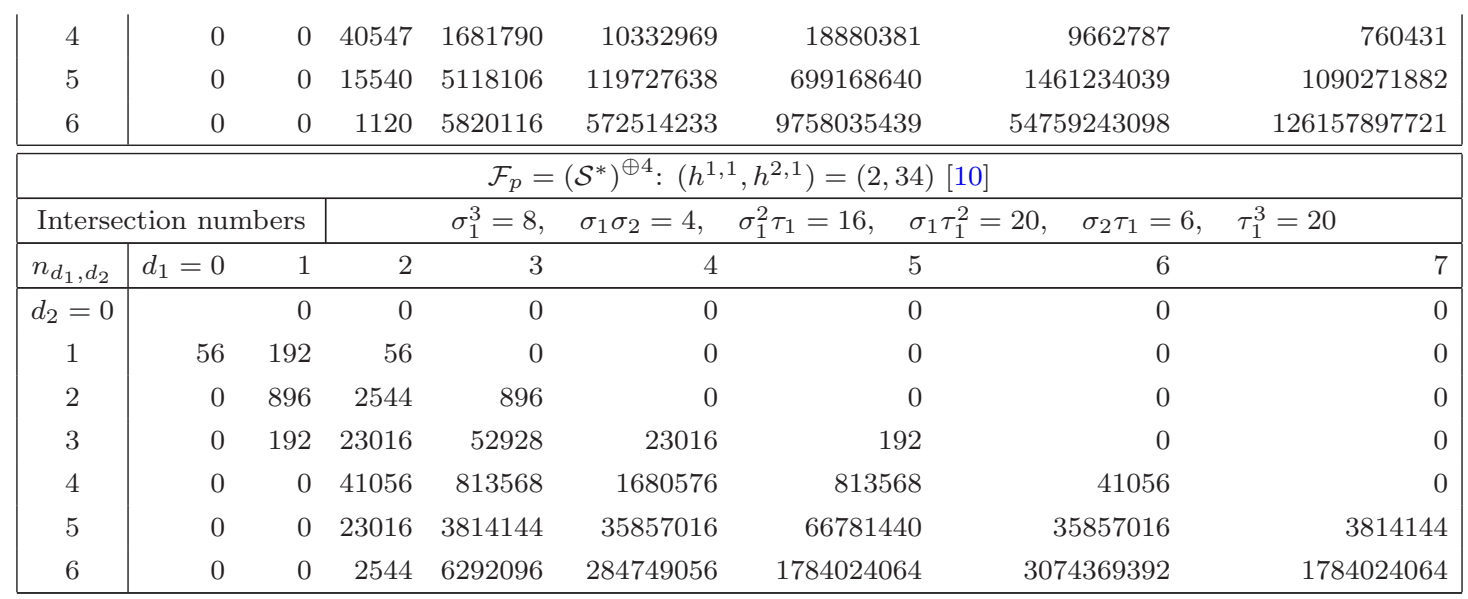

Table 6. Genus-0 invariants of determinantal 3-folds in $(2.25)$ with $V=G(2,4)$.

\section{Conclusions}

In this paper we have examined a class of square determinantal Calabi-Yau varieties in Grassmannians satisfying appropriate conditions about dimension, a Calabi-Yau definition, duality $G(k, n) \cong G(n-k, n)$, and rank of the vector bundles. We found that infinite families of examples associated with non-abelian quiver GLSMs might be possible. Furthermore, we explicitly demonstrated how to compute genus-0 integer invariants of the determinantal Calabi-Yau varieties via the Givental $I$-functions. By constructing the $I$ functions from the supersymmetric localization formula for the GLSM on a supersymmetric 2-sphere, we provided a guideline for the evaluation of the genus-0 A-model correlators. We also found the handy formula for the 1-point correlators for Grassmannian Calabi-Yau varieties, which turned out to be generalized into the cases with the determinantal varieties. We hope that our results would give a clue to understand various properties of the less studied GLSMs with non-abelian gauge groups.

Finally, we comment on possible future research directions.

- Since we have not imposed irreducibility as a requirement, to make our classification more rigorous, a comprehensive study of topological invariants such as Hodge numbers and Gromov-Witten invariants for the infinite families in (2.27), (A.4), and (A.10) is indispensable to check whether they are appropriate irreducible CalabiYau varieties.

- We have classified the square determinantal varieties based on the requirement (2.5). It would be interesting to examine determinantal varieties with general vector bundles $\mathcal{E}_{p}$ such as $\mathcal{E}_{p}=L \otimes \mathcal{O}_{V}^{\oplus p}$, where $L$ is a line bundle, as studied in [25].

- We conjectured the formula (3.24) for the genus-0 1-point A-model correlators for Grassmannian Calabi-Yau varieties, which generalizes the formula (3.16). It would be interesting to find out the 3-point extension of our formula (3.24) as a natural generalization of the formula (3.12) studied in [32], and give a proof of it. 
- In [52], GLSM realizations of the so-called Veronese embeddings and the Segre embeddings were proposed, and it opened up the possibility of more broad class of Calabi-Yau varieties. Various exotic Calabi-Yau examples including the constructions in [53] have also been discussed, and it would be interesting to consider these examples and discuss their $I$-functions.

\section{Acknowledgments}

The work of MM was supported by the ERC Starting Grant no. 335739 "Quantum fields and knot homologies" funded by the European Research Council under the European Union's Seventh Framework Programme, and by the Max-Planck-Institut für Mathematik in Bonn, and by the Australian Research Council.

\section{A Determinantal Calabi-Yau 2-folds and 4-folds}

In section 2.3, we have focused on the realization of a class of determinantal Calabi-Yau 3 -folds of square type. In a similar spirit, here we discuss the classification of determinantal Calabi-Yau 2-folds and 4-folds satisfying the requirements (2.7)-(2.10).

\section{A.1 Determinantal Calabi-Yau 2-folds}

When $\operatorname{dim} X_{A}=2$, the dimensional condition (2.7) becomes

$$
\ell_{p}^{\vee}\left(k \mathfrak{c}_{1}\left(\mathcal{F}_{p}\right)-\ell_{p}^{\vee}\right)=k^{2}+2
$$

Note that, as mentioned in Remark 2.1, the generic determinantal Calabi-Yau 2-folds $X_{A}$ do not have the singular loci. In the following, we clarify which type of choices for $\left(k, n ; \ell_{p}^{\vee}, \mathfrak{c}_{1}\left(\mathcal{F}_{p}\right)\right)$ can be possible while changing the parameter $k$.

\section{A.1.1 $k=1$}

When $k=1$ we have $V=G(1, n) \cong \mathbb{P}^{n-1}$, and the generic solution (2.15) with (2.16) provides a "quartic family" given by

$$
\left(k, n ; \ell_{p}^{\vee}, \mathfrak{c}_{1}\left(\mathcal{F}_{p}\right)\right)=(1,4 ; 1,4) \text { with } \mathcal{F}_{p}=\oplus_{i=1}^{r} \mathcal{O}_{V}\left(p_{i}\right), p_{1} \geq p_{2} \geq \cdots \geq p_{r}>0, \sum_{i=1}^{r} p_{i}=4
$$

In addition, (2.20) provides another class of solutions

$$
\left(k, n ; \ell_{p}^{\vee}, \mathfrak{c}_{1}\left(\mathcal{F}_{p}\right)\right)=(1,12 ; 3,4) \text { with } \mathcal{F}_{p}=\mathcal{O}_{V}(1)^{\oplus 2} \oplus \mathcal{O}_{V}(2), \mathcal{O}_{V}(1)^{\oplus 4}
$$

Here the Calabi-Yau 2-fold $X_{A}$ constructed from $\mathcal{F}_{p}=\mathcal{O}_{V}(1)^{\oplus 2} \oplus \mathcal{O}_{V}(2)$ with $p=3$ (i.e. $\ell=0$ ) can be described by the complete intersection Calabi-Yau 2-fold in $\mathbb{P}^{5}$ with the vector bundle $\mathcal{O}_{\mathbb{P}^{5}}(2)^{\oplus 3}$ as

$$
X_{A} \text { with } \mathcal{O}_{V}(1)^{\oplus 2} \oplus \mathcal{O}_{V}(2) \longleftrightarrow X_{2,2,2} \subset \mathbb{P}^{5} .
$$




\section{A.1.2 $k \geq 2$}

When $k \geq 2$ we have $V=G(k, n)$. In this case, there exist two "infinite families" of solutions as determinantal 2-folds satisfying all the requirements (A.1), (2.8), (2.9), and (2.10).

$$
\left(k, n ; \ell_{p}^{\vee}, \mathfrak{c}_{1}\left(\mathcal{F}_{p}\right)\right)=\left(k_{i}, 4 \ell_{i} ; \ell_{i}, 4\right), \quad i \in \mathbb{N}
$$

where $k_{1}=3, \ell_{1}=11, k_{i+1}=\ell_{i}, \ell_{i+1}=-k_{i}+4 \ell_{i}$ with $\mathcal{F}_{p}=\left(\mathcal{S}^{*}\right)^{\oplus 4}, \mathcal{Q}^{\oplus 4}$.

\section{A.2 Determinantal Calabi-Yau 4-folds}

When $\operatorname{dim} X_{A}=4$, the dimensional condition (2.7) becomes

$$
\ell_{p}^{\vee}\left(k \mathfrak{c}_{1}\left(\mathcal{F}_{p}\right)-\ell_{p}^{\vee}\right)=k^{2}+4
$$

\section{A.2.1 $k=1$}

When $k=1$ we have $V=G(1, n) \cong \mathbb{P}^{n-1}$, and the generic solution (2.15) with (2.16) provides a "sextic family" given by

$\left(k, n ; \ell_{p}^{\vee}, \mathfrak{c}_{1}\left(\mathcal{F}_{p}\right)\right)=(1,6 ; 1,6)$ with $\mathcal{F}_{p}=\oplus_{i=1}^{r} \mathcal{O}_{V}\left(p_{i}\right), p_{1} \geq p_{2} \geq \cdots \geq p_{r}>0, \sum_{i=1}^{r} p_{i}=6$

Via the incidence correspondence (2.6), the sextic family is connected each other through the extremal transitions (see appendix C.1).

In addition, (2.20) provides another class of solutions

$$
\left(k, n ; \ell_{p}^{\vee}, \mathfrak{c}_{1}\left(\mathcal{F}_{p}\right)\right)=(1,30 ; 5,6) \text { with } \mathcal{F}_{p}=\mathcal{O}_{V}(1)^{\oplus 4} \oplus \mathcal{O}_{V}(2), \mathcal{O}_{V}(1)^{\oplus 6}
$$

Here the Calabi-Yau 4-fold $X_{A}$ constructed from $\mathcal{F}_{p}=\mathcal{O}_{V}(1)^{\oplus 4} \oplus \mathcal{O}_{V}(2)$ with $p=5$ (i.e. $\ell=0)$ can be described by the complete intersection Calabi-Yau 4 -fold in $\mathbb{P}^{9}$ with the vector bundle $\mathcal{O}_{\mathbb{P}^{5}}(2)^{\oplus 5}$ as

$$
X_{A} \text { with } \mathcal{O}_{V}(1)^{\oplus 4} \oplus \mathcal{O}_{V}(2) \longleftrightarrow X_{2,2,2,2,2} \subset \mathbb{P}^{9} .
$$

\section{A.2.2 $k=2$}

When $k=2$ we have $V=G(2, n)$. In this case there exists a class of determinantal 4-folds which satisfy the conditions (A.5), (2.8), (2.9), and (2.10) given $\mathrm{by}^{7}$

$$
\begin{aligned}
(k, n ; & \left.\ell_{p}^{\vee}, \mathfrak{c}_{1}\left(\mathcal{F}_{p}\right)\right)=(2,6 ; 2,3) \text { with } \\
\mathcal{F}_{p}= & \mathcal{O}_{V}(1) \oplus \mathcal{O}_{V}(2), \mathcal{S}^{*}(1), \mathcal{O}_{V}(1)^{\oplus 3}, \mathcal{S}^{*} \oplus \mathcal{O}_{V}(2), \operatorname{Sym}^{2} \mathcal{S}^{*}, \mathcal{S}^{*} \oplus \mathcal{O}_{V}(1)^{\oplus 2} \\
& \left(\mathcal{S}^{*}\right)^{\oplus 2} \oplus \mathcal{O}_{V}(1),\left(\mathcal{S}^{*}\right)^{\oplus 3}, \mathcal{Q} \oplus \mathcal{O}_{V}(2), \mathcal{Q} \oplus \mathcal{O}_{V}(1)^{\oplus 2}, \mathcal{Q}^{\oplus 2} \oplus \mathcal{O}_{V}(1), \mathcal{Q}^{\oplus 3} \\
& \wedge^{2} \mathcal{Q}, \wedge^{3} \mathcal{Q}, \mathcal{S}^{*} \oplus \mathcal{Q} \oplus \mathcal{O}_{V}(1),\left(\mathcal{S}^{*}\right)^{\oplus 2} \oplus \mathcal{Q}, \mathcal{S}^{*} \oplus \mathcal{Q}^{\oplus 2} .
\end{aligned}
$$

\footnotetext{
${ }^{7}$ In appendix C.2, we see that the determinantal 4-fold associated with $\mathcal{F}_{p}=\operatorname{Sym}^{2} \mathcal{S}^{*}$ is not a Calabi-Yau variety with $\left(h^{1,0}, h^{2,0}\right)=(0,0)$ but an irreducible holomorphic symplectic variety with $\left(h^{1,0}, h^{2,0}\right)=(0,1)$.
} 
Here the Calabi-Yau 4-fold $X_{A}$ constructed from $\mathcal{F}_{p}=\mathcal{O}_{V}(1) \oplus \mathcal{O}_{V}(2)\left(\right.$ resp. $\mathcal{S}^{*}(1)$ ) with $p=2$ (i.e. $\ell=0$ ) can be described by the complete intersection Grassmannian Calabi-

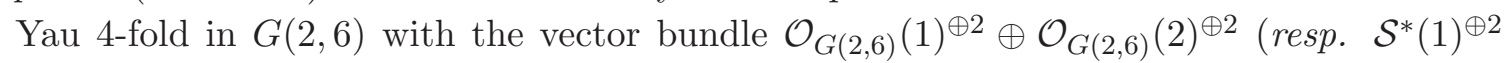
on $G(2,6))$.

We find that there exist another type of solutions satisfying all the requirements (A.5), (2.8), (2.9), and (2.10) given by

$$
\begin{gathered}
\left(k, n ; \ell_{p}^{\vee}, \mathfrak{c}_{1}\left(\mathcal{F}_{p}\right)\right)=(2,12 ; 4,3) \text { with } \\
\mathcal{F}_{p}=\mathcal{S}^{*} \oplus \mathcal{O}_{V}(1)^{\oplus 2},\left(\mathcal{S}^{*}\right)^{\oplus 2} \oplus \mathcal{O}_{V}(1),\left(\mathcal{S}^{*}\right)^{\oplus 3}, \mathcal{Q} \oplus \mathcal{O}_{V}(2), \\
\quad \mathcal{Q} \oplus \mathcal{O}_{V}(1)^{\oplus 2}, \mathcal{Q}^{\oplus 2} \oplus \mathcal{O}_{V}(1), \mathcal{Q}^{\oplus 3} .
\end{gathered}
$$

Here the Calabi-Yau 4-fold $X_{A}$ constructed from $\mathcal{F}_{p}=\mathcal{S}^{*} \oplus \mathcal{O}_{V}(1)^{\oplus 2}$ with $p=4$ (i.e. $\ell=0$ ) can be described by the complete intersection Grassmannian Calabi-Yau 4-fold in $G(2,8)$ with the vector bundle $\mathcal{O}_{G(2,8)}(1)^{\oplus 8}$.

\section{A.2.3 $k \geq 3$}

When $k \geq 3$ we have $V=G(k, n)$, and there exist six "infinite families" of determinantal 4 -folds given by

$$
\begin{aligned}
\left(k, n ; \ell_{p}^{\vee}, \mathfrak{c}_{1}\left(\mathcal{F}_{p}\right)\right) & =\left(k_{i}, 6 \ell_{i} ; \ell_{i}, 6\right), \quad i \in \mathbb{N} \\
\text { with } k_{1}=5, \ell_{1} & =29, k_{i+1}=\ell_{i}, \ell_{i+1}=-k_{i}+6 \ell_{i}: \mathcal{F}_{p}=\left(\mathcal{S}^{*}\right)^{\oplus 6}, \mathcal{Q}^{\oplus 6}, \\
\left(k, n ; \ell_{p}^{\vee}, \mathfrak{c}_{1}\left(\mathcal{F}_{p}\right)\right) & =\left(k_{i}, 3 \ell_{i} ; \ell_{i}, 3\right), \quad i \in \mathbb{N} \\
\text { with } k_{1}=4, \ell_{1} & =10, k_{i+1}=-3 k_{i}+8 \ell_{i}, \ell_{i+1}=-8 k_{i}+21 \ell_{i}: \mathcal{F}_{p}=\left(\mathcal{S}^{*}\right)^{\oplus 3}, \mathcal{Q}^{\oplus 3}, \\
\left(k, n ; \ell_{p}^{\vee}, \mathfrak{c}_{1}\left(\mathcal{F}_{p}\right)\right) & =\left(k_{i}, 3 \ell_{i} ; \ell_{i}, 3\right), \quad i \in \mathbb{N} \\
\text { with } k_{1}=10, \ell_{1} & =26, k_{i+1}=-3 k_{i}+8 \ell_{i}, \ell_{i+1}=-8 k_{i}+21 \ell_{i}: \mathcal{F}_{p}=\left(\mathcal{S}^{*}\right)^{\oplus 3}, \mathcal{Q}^{\oplus 3},
\end{aligned}
$$

where the third (resp. fourth) and the fifth (resp. sixth) families of 4 -folds associated with $\mathcal{F}_{p}=\left(\mathcal{S}^{*}\right)^{\oplus 3}\left(\right.$ resp. $\left.\mathcal{Q}^{\oplus 3}\right)$ are given by the same recurrence relation with the different initial conditions. By using mathematical induction, one can check that the duality condition (2.9), the rank condition (2.10), and in particular $\ell_{p}^{\vee}<\operatorname{rank} \mathcal{F}_{p}$, are maintained.

\section{B Hodge number calculations via the Koszul complex}

Following [54] (see also e.g. [55-58]), here we briefly review how to compute cohomologies and Hodge numbers of Calabi-Yau varieties via the Koszul complex. We will demonstrate the explicit computations for several examples.

\section{B.1 General algorithm}

Let $V$ be a complex manifold, $\mathcal{E}_{p}$ be a rank $p$ vector bundle over $V$ and consider the locus $X \subset V$ as a holomorphic section of $\mathcal{E}_{p}$. In this appendix we describe how to compute the cohomologies

$$
H^{\operatorname{dim} X-1, i}(X)=H^{i}(X, T X), \quad \text { or } \quad H^{1, i}(X)=H^{i}\left(X, T^{*} X\right), \quad i=0,1, \ldots, \operatorname{dim} X,
$$

via the Koszul complex. 


\section{B.1.1 Step 1: computation of (B.2)}

First we describe a method to compute bundle-valued cohomologies of $X$,

$$
H^{i}\left(X,\left.\mathcal{F}_{V}\right|_{X}\right),
$$

by using the Koszul exact sequence.

The Koszul exact sequence gives the resolution of $\mathcal{O}_{X}$ over $V$ as

$$
0 \longrightarrow \wedge^{p} \mathcal{E}_{p}^{*} \longrightarrow \cdots \longrightarrow \wedge^{2} \mathcal{E}_{p}^{*} \longrightarrow \mathcal{E}_{p}^{*} \longrightarrow \mathcal{O}_{V} \longrightarrow \mathcal{O}_{X} \longrightarrow 0 .
$$

For the Koszul exact sequence, the Koszul spectral sequence (see e.g. [42]),

$$
\left\{E_{r}^{i, q}\right\}, \quad i=0,1, \ldots, \operatorname{dim} V, \quad q=0,1, \ldots, p, \quad r=0,1,2, \ldots,
$$

can be associated as follows. Starting from

$$
E_{0}^{i, q}=H^{i}\left(V, \wedge^{q} \mathcal{E}_{p}^{*}\right),
$$

define $d_{r}$-cohomology recursively as

$$
E_{r+1}^{i, q}=\frac{\operatorname{ker}\left(d_{r}: E_{r}^{i, q} \longrightarrow E_{r}^{i-r, q-r-1}\right)}{\operatorname{im}\left(d_{r}: E_{r}^{i+r, q+r+1} \longrightarrow E_{r}^{i, q}\right)}
$$

which is associated with differentials

$$
d_{r}: E_{r}^{i, q} \longrightarrow E_{r}^{i-r, q-r-1},
$$

with $d_{r} \circ d_{r}=0$. Here $E_{r}^{i, q}=0$ for $i, q<0, i>\operatorname{dim} V$, and $q>p$. At finite $r=r_{0}, E_{r}^{i, q}$ converges to $E_{r_{0}}^{i, q}=E_{r_{0}+1}^{i, q}=\ldots=E_{\infty}^{i, q}$ and we obtain a cohomology of $X$ as

$$
\sum_{q=0}^{p} E_{\infty}^{i+q, q} \Longrightarrow H^{i}\left(X, \mathcal{O}_{X}\right)=H^{0, i}(X),
$$

where the summation represents a formal sum.

Remark B.1. By tensoring the Koszul exact sequence (B.3) with a vector bundle $\mathcal{F}_{V}$ (e.g. $\mathcal{E}_{p}, \mathcal{E}_{p}^{*}, T V, T^{*} V$, etc.) over $V$, one obtains the resolution of $\left.\mathcal{F}_{V}\right|_{X}$ over $V$ as

$$
\left.0 \longrightarrow \wedge^{p} \mathcal{E}_{p}^{*} \otimes \mathcal{F}_{V} \longrightarrow \cdots \longrightarrow \wedge^{2} \mathcal{E}_{p}^{*} \otimes \mathcal{F}_{V} \longrightarrow \mathcal{E}_{p}^{*} \otimes \mathcal{F}_{V} \longrightarrow \mathcal{F}_{V} \longrightarrow \mathcal{F}_{V}\right|_{X} \longrightarrow 0 .
$$

Then, by considering the Koszul spectral sequence associated with (B.8), one can obtain the bundle-valued cohomologies $H^{i}\left(X,\left.\mathcal{F}_{V}\right|_{X}\right)$ in (B.2).

Therefore, by using the Koszul spectral sequence, the cohomologies $H^{i}\left(X,\left.\mathcal{F}_{V}\right|_{X}\right)$ can be computed from the cohomologies $H^{i}\left(V, \wedge^{q} \mathcal{E}_{p}^{*} \otimes \mathcal{F}_{V}\right)$. For computing these quantities, the Bott-Borel-Weil theorem B.5 is quite useful. To state the theorem, consider a flag manifold

$$
V=\frac{\mathrm{U}(N)}{\mathrm{U}\left(n_{1}\right) \times \cdots \times \mathrm{U}\left(n_{F}\right)}, \quad N=\sum_{i=1}^{F} n_{i} .
$$


A holomorphic homogeneous vector bundle $\mathcal{F}_{V}$ over $V$ can be described by a representation of $\mathrm{U}\left(n_{1}\right) \times \cdots \times \mathrm{U}\left(n_{F}\right)$, where a representation of each $\mathrm{U}(n)$ is described by a Young diagram which is given by a monotonically increasing sequence with length $n$ of integers as $\left(a_{1}, \ldots, a_{n}\right), a_{i} \leq a_{i+1}$. Then, a vector bundle $\mathcal{F}_{V}$ is described by a representation of $\mathrm{U}\left(n_{1}\right) \times \cdots \times \mathrm{U}\left(n_{F}\right)$ as

$$
\mathcal{F}_{V} \sim\left(a_{1}, \ldots, a_{n_{1}}\left|b_{1}, \ldots, b_{n_{2}}\right| \cdots \mid r_{1}, \ldots, r_{n_{F}}\right) .
$$

Example B.2. For $V=\mathbb{P}^{n-1}=\mathrm{U}(n) /[\mathrm{U}(1) \times \mathrm{U}(n-1)]$, in terms of the representations of $\mathrm{U}(1) \times \mathrm{U}(n-1)$, one can describe e.g.,

$$
\begin{aligned}
\mathcal{S} & \sim(1 \mid 0, \ldots, 0), & \mathcal{S}^{*} & \sim(-1 \mid 0, \ldots, 0), \\
\mathcal{O}_{V}(p) & =\left(\mathcal{S}^{*}\right)^{\otimes p} \sim(-p \mid 0, \ldots, 0), & \mathcal{O}_{V}(p)^{*} & =\mathcal{S}^{\otimes p} \sim(p \mid 0, \ldots, 0), \\
T V & \sim(-1 \mid 0, \ldots, 0,1), & T^{*} V & \sim(1 \mid-1,0, \ldots, 0),
\end{aligned}
$$

where $\mathcal{S}$ is the universal subbundle on $V=\mathbb{P}^{n-1}$. The representations of tensor product and wedge product are obtained as e.g.,

$$
\mathcal{O}_{V}(p)^{*} \otimes T^{*} V \sim(p+1 \mid-1,0, \ldots, 0), \quad \wedge^{2} T V \sim(-2 \mid 0, \ldots, 0,1,1) .
$$

Example B.3. For $V=G(k, n)=\mathrm{U}(n) /[\mathrm{U}(k) \times \mathrm{U}(n-k)]$, in terms of the representations of $\mathrm{U}(k) \times \mathrm{U}(n-k)$, one can describe e.g.,

$$
\begin{aligned}
\mathcal{S} & \sim(0, \ldots, 0,1 \mid 0, \ldots, 0), \quad \mathcal{S}^{*} \sim(-1,0, \ldots, 0 \mid 0, \ldots, 0), \\
\mathcal{O}_{V}(p) & =\left(\operatorname{det} \mathcal{S}^{*}\right)^{\otimes p} \sim(-p, \ldots,-p \mid 0, \ldots, 0), \\
\mathcal{O}_{V}(p)^{*} & =(\operatorname{det} \mathcal{S})^{\otimes p} \sim(p, \ldots, p \mid 0, \ldots, 0), \\
T V & \sim(-1,0, \ldots, 0 \mid 0, \ldots, 0,1), \quad T^{*} V \sim(0, \ldots, 0,1 \mid-1,0, \ldots, 0),
\end{aligned}
$$

where $\mathcal{S}$ is the rank $k$ universal subbundle on $V=G(k, n)$. The representations of tensor product and wedge product are obtained e.g. for $V=G(2,5)$ as

$$
\begin{aligned}
& \mathcal{S}(-1)=\mathcal{S} \otimes \mathcal{O}_{V}(1)^{*} \sim(1,2 \mid 0, \ldots, 0), \quad \wedge^{2} \mathcal{S}(-1) \sim(3,3 \mid 0, \ldots, 0,0), \\
& \mathcal{S}(-1) \wedge \mathcal{O}_{V}(2)^{*} \sim(3,4 \mid 0, \ldots, 0,0), \quad\left(\wedge^{2} \mathcal{S}(-1)\right) \wedge \mathcal{O}_{V}(2)^{*} \sim(5,5 \mid 0, \ldots, 0,0) .
\end{aligned}
$$

Example B.4. For product manifold $V=\mathbb{P}^{2} \times G(2,4)$, one obtains e.g.,

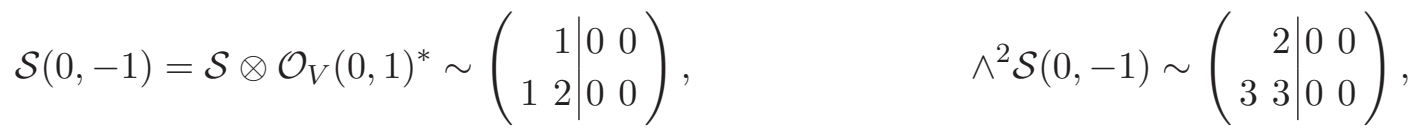

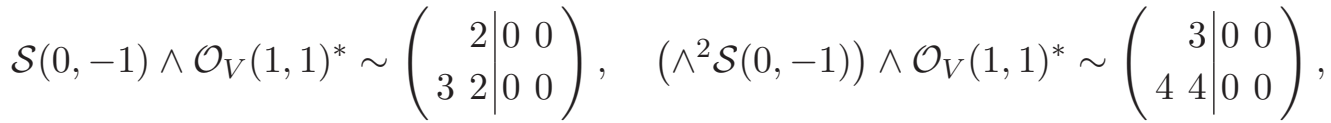

where $\mathcal{S}$ is the rank $(1,2)$ universal subbundle on $V$.

Using the above representations (B.10) for vector bundles, the Bott-Borel-Weil theorem is stated as follows. 
Theorem B.5 (Bott-Borel-Weil). Let $\mathcal{F}_{V}$ be a holomorphic homogeneous vector bundle, represented as (B.10), over a flag manifold $V$ in (B.9). Then at most only one of the cohomologies $H^{i}\left(V, \mathcal{F}_{V}\right)$ is non-trivial $\left(\cong \mathbb{C}^{D}\right)$, and $D$ is given by the dimension of an irreducible representation $\left(y_{1}, \ldots, y_{N}\right)$ of $\mathrm{U}(N)$ determined as follows:

1. For the sequence (B.10), add the sequence $(1,2, \ldots, N)$ as

$$
\left(a_{1}+1, a_{2}+2, \ldots, a_{n_{1}}+n_{1}, b_{1}+n_{1}+1, \ldots, b_{n_{2}}+n_{1}+n_{2}, \ldots, r_{n_{F}}+N\right) .
$$

2. If the above sequence contains any same number, the cohomologies $H^{i}\left(V, \mathcal{F}_{V}\right)$ are trivial, if not;

3. Minimally swap the above sequence, with the minimal swapping number $I$, so that the resulting sequence gives a strictly increasing sequence $\left(y_{1}^{\prime}, \ldots, y_{N}^{\prime}\right), y_{i}^{\prime}<y_{i+1}^{\prime}$.

4. For the above swapped sequence, subtracting the sequence $(1,2, \ldots, N)$ as

$$
\left(y_{1}, y_{2}, \ldots, y_{N}\right)=\left(y_{1}^{\prime}-1, y_{2}^{\prime}-2, \ldots, y_{N}^{\prime}-N\right)
$$

one obtains a representation $\left(y_{1}, \ldots, y_{N}\right)$ of $\mathrm{U}(N)$ which gives $H^{I}\left(V, \mathcal{F}_{V}\right)$.

Remark B.6. The dimension $D$ of a representation $\left(y_{1}, \ldots, y_{N}\right)$ of $\mathrm{U}(N)$, which is given by a Young diagram $Y$ with length $y_{i}$ for the $i$-th row, is computed by

$$
D=\prod_{s \in Y} \frac{F_{Y}(s)}{H_{Y}(s)}
$$

where $H_{Y}(s)$ is the hook length of $s$ in $Y$, and $F_{Y}(s)=N-i+j$ for $s=(i, j)$ (the box of $i$-th row and $j$-th column).

Remark B.7. When $V$ is a product manifold $V=V_{1} \times V_{2}$ of two flag manifolds $V_{1}$ and $V_{2}$, for computing the cohomologies of $V$ one can use the Künneth formula

$$
H^{i}\left(V, \mathcal{F}_{V}\right)=\bigoplus_{i_{1}+i_{2}=i} H^{i_{1}}\left(V_{1},\left.\mathcal{F}_{V}\right|_{V_{1}}\right) \otimes H^{i_{2}}\left(V_{2},\left.\mathcal{F}_{V}\right|_{V_{2}}\right)
$$

where $\mathcal{F}_{V}$ is a vector bundle over $V$ and each $\left.\mathcal{F}_{V}\right|_{V_{i}}$ is the restricted vector bundle over $V_{i}$.

\section{B.1.2 Step 2: computation of (B.1)}

By Step 1 one can compute, in particular, $H^{0, i}(X)=H^{i}\left(X, \mathcal{O}_{X}\right), H^{i}\left(X,\left.T V\right|_{X}\right)$, $H^{i}\left(X,\left.\mathcal{E}_{p}\right|_{X}\right), H^{i}\left(X,\left.T^{*} V\right|_{X}\right)$, and $H^{i}\left(X,\left.\mathcal{E}_{p}^{*}\right|_{X}\right)$. Now, using these results one can compute the cohomologies (B.1) via the short exact sequence (adjunction formula)

$$
\left.\left.0 \longrightarrow T X \longrightarrow T V\right|_{X} \longrightarrow \mathcal{E}_{p}\right|_{X} \longrightarrow 0
$$

or its dual

$$
\left.\left.0 \longrightarrow \mathcal{E}_{p}^{*}\right|_{X} \longrightarrow T^{*} V\right|_{X} \longrightarrow T^{*} X \longrightarrow 0
$$


The former and the latter in (B.1) are related by the Hodge dual, and then in the following we only describe the computation of the latter by using the exact sequence (B.16). The exact sequence (B.16) induces the following long exact sequence:

$$
\begin{aligned}
& 0 \longrightarrow \quad H^{0}\left(X,\left.\mathcal{E}_{p}^{*}\right|_{X}\right) \longrightarrow \quad H^{0}\left(X,\left.T^{*} V\right|_{X}\right) \longrightarrow \quad H^{0}\left(X, T^{*} X\right) \longrightarrow \\
& \longrightarrow \quad H^{1}\left(X,\left.\mathcal{E}_{p}^{*}\right|_{X}\right) \longrightarrow \quad H^{1}\left(X,\left.T^{*} V\right|_{X}\right) \longrightarrow \quad H^{1}\left(X, T^{*} X\right) \longrightarrow \ldots \\
& \ldots \longrightarrow H^{\operatorname{dim} X}\left(X,\left.\mathcal{E}_{p}^{*}\right|_{X}\right) \longrightarrow H^{\operatorname{dim} X}\left(X,\left.T^{*} V\right|_{X}\right) \longrightarrow H^{\operatorname{dim} X}\left(X, T^{*} X\right) \longrightarrow 0 \text {. }
\end{aligned}
$$

Then, using the above exact sequence, from the cohomologies $H^{i}\left(X,\left.T^{*} V\right|_{X}\right)$ and $H^{i}\left(X,\left.\mathcal{E}_{p}^{*}\right|_{X}\right)$ obtained in Step 1 , one can compute the cohomologies $H^{i}\left(X, T^{*} X\right)$.

Remark B.8. To find other cohomologies, one can use the following well-known relations

$$
\begin{aligned}
\text { Complex conjugate: } & & H^{i, j}(X) \cong H^{j, i}(X), \\
\text { Hodge duality: } & & H^{i, j}(X) \cong H^{\operatorname{dim} X-i, \operatorname{dim} X-j}(X) .
\end{aligned}
$$

Furthermore, if $X$ admits the unique holomorphic 3-form in $H^{\operatorname{dim} X, 0}(X)=\mathbb{C}$, Serre duality gives $H^{0, i}(X) \cong H^{\operatorname{dim} X, i}(X)$.

Remark B.9. To compute the Hodge numbers of $X$, one can also use a formula for the Hirzebruch $\chi_{y}$-genus (see [59] for the explicit formulae of $\operatorname{dim} X=2,3,4$ written in terms of the Chern classes of $X)$ :

$$
\chi_{y}=\sum_{i, j=0}^{\operatorname{dim} X}(-1)^{j} \operatorname{dim} H^{j}\left(X, \wedge^{i} T^{*} X\right) y^{i}=\int_{X} \prod_{k=1}^{p} \frac{\left(1+y \mathrm{e}^{-x_{k}}\right) x_{k}}{1-\mathrm{e}^{-x_{k}}},
$$

which is derived from Hirzebruch-Riemann-Roch index theorem. Here $x_{k}, k=1, \ldots, p$, are the Chern roots of $X$ in (3.1).

\section{B.2 Examples}

We demonstrate the explicit computations of cohomologies for some examples based on the strategy in appendix B.1.

\section{B.2.1 Quintic Calabi-Yau 3-fold: $\mathcal{E}_{1}=\mathcal{O}_{V}(5)$ on $V=\mathbb{P}^{4}$}

As a famous example, consider the quintic Calabi-Yau 3-fold $X$ defined by the zero locus of a holomorphic section of $\mathcal{E}_{1}=\mathcal{O}_{V}(5)$ on $V=\mathbb{P}^{4}$ [36]. Using the representations in Example B.2, the Koszul exact sequence (B.3) is given by

$$
0 \longrightarrow(5 \mid 0,0,0,0) \longrightarrow(0 \mid 0,0,0,0) \longrightarrow \mathcal{O}_{X} \longrightarrow 0 .
$$

By (B.7) and the Bott-Borel-Weil theorem B.5, one finds that

$$
H^{0}\left(V, \mathcal{O}_{V}\right)=\mathbb{C} \Longrightarrow H^{0,0}(X)=\mathbb{C}, \quad H^{4}\left(V, \mathcal{O}_{V}(5)^{*}\right)=\mathbb{C} \Longrightarrow H^{0,3}(X)=\mathbb{C},
$$

and $H^{0,1}(X)$ and $H^{0,2}(X)$ are trivial, i.e. $H^{0,1}(X)=H^{0,2}(X)=0$. 
For $\mathcal{F}_{V}=\mathcal{O}_{V}(5)^{*}$ and $\mathcal{F}_{V}=T^{*} V$ the exact sequence (B.8) yields

$$
\begin{aligned}
& \left.0 \longrightarrow(10 \mid 0,0,0,0) \longrightarrow(5 \mid 0,0,0,0) \longrightarrow \mathcal{O}_{V}(5)^{*}\right|_{X} \longrightarrow 0 \\
& \left.0 \longrightarrow(6 \mid-1,0,0,0) \longrightarrow(1 \mid-1,0,0,0) \longrightarrow T^{*} V\right|_{X} \longrightarrow 0 .
\end{aligned}
$$

From (B.21) one finds that

$$
\begin{aligned}
\operatorname{ker}\left(d_{0}: H^{4}\left(V, \mathcal{O}_{V}(5)^{*} \otimes \mathcal{O}_{V}(5)^{*}\right)\right. & \left.=\mathbb{C}^{126} \longrightarrow H^{4}\left(V, \mathcal{O}_{V}(5)^{*}\right)=\mathbb{C}\right) \\
\Longrightarrow H^{3}\left(X,\left.\mathcal{O}_{V}(5)^{*}\right|_{X}\right) & =\mathbb{C}^{125}
\end{aligned}
$$

and $H^{i}\left(X,\left.\mathcal{O}_{V}(5)^{*}\right|_{X}\right), i=0,1,2$, are trivial. From (B.22) one finds that

$$
\begin{aligned}
H^{1}\left(V, T^{*} V\right) & =\mathbb{C} \Longrightarrow H^{1}\left(X,\left.T^{*} V\right|_{X}\right)=\mathbb{C} \\
H^{4}\left(V, \mathcal{O}_{V}(5)^{*} \otimes T^{*} V\right) & =\mathbb{C}^{24} \Longrightarrow H^{3}\left(X,\left.T^{*} V\right|_{X}\right)=\mathbb{C}^{24},
\end{aligned}
$$

and $H^{0}\left(X,\left.T^{*} V\right|_{X}\right)$ and $H^{2}\left(X,\left.T^{*} V\right|_{X}\right)$ are trivial. Using these results, the exact sequence (B.17) yields the following exact sequences:

$$
\begin{aligned}
& 0 \longrightarrow H^{0}\left(X, T^{*} X\right) \longrightarrow 0, \\
& 0 \longrightarrow \mathbb{C} \longrightarrow H^{1}\left(X, T^{*} X\right) \longrightarrow 0, \\
& 0 \longrightarrow H^{2}\left(X, T^{*} X\right) \longrightarrow \mathbb{C}^{125} \stackrel{f}{\longrightarrow} \mathbb{C}^{24} \longrightarrow H^{3}\left(X, T^{*} X\right) \longrightarrow 0 .
\end{aligned}
$$

Then one obtains

$$
\begin{array}{ll}
H^{1,0}(X)=H^{0}\left(X, T^{*} X\right)=0, & H^{1,1}(X)=H^{1}\left(X, T^{*} X\right)=\mathbb{C}, \\
H^{1,2}(X)=H^{2}\left(X, T^{*} X\right)=\operatorname{ker}(f)=\mathbb{C}^{101}, & H^{1,3}(X)=H^{3}\left(X, T^{*} X\right)=\operatorname{coker}(f)=0 .
\end{array}
$$

As a result, the Hodge diamond is obtained as

$$
\begin{array}{ccccccccccccccc} 
& & h^{0,0} & & & & & & & 1 & & \\
& h^{1,0} & & h^{0,1} & & & & & 0 & & 0 & & \\
h^{2,0} & & h^{1,1} & & h^{0,2} & & & 0 & & 1 & & 0 & \\
& h^{2,1} & & h^{1,2} & & h^{0,3}=1 & & 101 & & 101 & & 1 \\
h^{3,1} & & h^{2,2} & & h^{1,3} & & & 0 & & 1 & & 0 & \\
& h^{3,2} & & h^{2,3} & & & & & 0 & & 0 & & \\
& & h^{3,3} & & & & & & & 1 & & &
\end{array}
$$

B.2.2 Grassmannian Calabi-Yau 3-fold: $\mathcal{E}_{3}=\mathcal{S}^{*}(1) \oplus \mathcal{O}_{V}(2)$ on $V=G(2,5)$

As a non-abelian example, consider a Grassmannian Calabi-Yau 3-fold $X$ defined by the zero locus of a holomorphic section of $\mathcal{E}_{3}=\mathcal{S}^{*}(1) \oplus \mathcal{O}_{V}(2)$ on $V=G(2,5)$ [60, 61]. Using the representations in Example B.3, the Koszul exact sequence (B.3) is given by

$$
0 \rightarrow(5,5 \mid 0,0,0) \rightarrow \underset{(3,4 \mid 0,0,0)}{\oplus} \rightarrow \underset{(2,2 \mid 0,0,0)}{\oplus} \rightarrow(0,0 \mid 0,0,0) \rightarrow \mathcal{O}_{X} \rightarrow 0
$$


By (B.7) and the Bott-Borel-Weil theorem B.5, one finds that

$$
H^{0}\left(V, \mathcal{O}_{V}\right)=\mathbb{C} \Longrightarrow H^{0,0}(X)=\mathbb{C}, \quad H^{6}\left(V, \wedge^{3} \mathcal{E}_{3}^{*}\right)=\mathbb{C} \Longrightarrow H^{0,3}(X)=\mathbb{C},
$$

and $H^{0,1}(X)$ and $H^{0,2}(X)$ are trivial, i.e. $H^{0,1}(X)=H^{0,2}(X)=0$.

For $\mathcal{F}_{V}=\mathcal{S}(1), \mathcal{O}_{V}(2)^{*}$, and $T^{*} V$ the exact sequence (B.8) yields respectively,

$$
\begin{aligned}
& (4,5 \mid 0,0,0) \quad(2,4 \mid 0,0,0) \\
& \oplus \quad \oplus \\
& \left.0 \rightarrow(6,7 \mid 0,0,0) \rightarrow(5,5 \mid 0,0,0) \rightarrow(3,3 \mid 0,0,0) \rightarrow(1,2 \mid 0,0,0) \rightarrow \mathcal{S}(1)\right|_{X} \rightarrow 0, \\
& \oplus \oplus \\
& (4,6 \mid 0,0,0) \quad(3,4 \mid 0,0,0) \\
& (5,5 \mid 0,0,0) \quad(3,4 \mid 0,0,0) \\
& \left.0 \rightarrow(7,7 \mid 0,0,0) \rightarrow \underset{(5,6 \mid 0,0,0)}{\oplus} \rightarrow \underset{(4,4 \mid 0,0,0)}{\oplus} \rightarrow(2,2 \mid 0,0,0) \rightarrow \mathcal{O}(2)^{*}\right|_{X} \rightarrow 0,
\end{aligned}
$$

and

$$
\begin{array}{cc}
(3,4 \mid-1,0,0) & (1,3 \mid-1,0,0) \\
\oplus & \oplus \\
\oplus \rightarrow(5,6 \mid-1,0,0) \rightarrow(3,5 \mid-1,0,0) & \left.\rightarrow(2,2 \mid-1,0,0) \rightarrow(0,1 \mid-1,0,0) \rightarrow T^{*} V\right|_{X} \rightarrow 0 . \\
\oplus & \oplus
\end{array}
$$

From (B.29) one finds that

$$
\begin{aligned}
\operatorname{ker}\left(d_{0}: H^{6}\left(V, \wedge^{3} \mathcal{E}_{3}^{*} \otimes \mathcal{S}(1)\right)\right. & \left.=\mathbb{C}^{40} \longrightarrow H^{6}\left(V, \wedge^{2} \mathcal{E}_{3}^{*} \otimes \mathcal{S}(1)\right)=\mathbb{C}\right) \\
\Longrightarrow H^{3}\left(X,\left.\mathcal{S}(1)\right|_{X}\right) & =\mathbb{C}^{39},
\end{aligned}
$$

and $H^{i}\left(X,\left.\mathcal{S}(1)\right|_{X}\right), i=0,1,2$, are trivial. From (B.30) one finds that

$$
\begin{aligned}
\operatorname{ker}\left(d_{0}: H^{6}\left(V, \wedge^{3} \mathcal{E}_{3}^{*} \otimes \mathcal{O}_{V}(2)^{*}\right)\right. & \left.=\mathbb{C}^{50} \longrightarrow H^{6}\left(V, \wedge^{2} \mathcal{E}_{3}^{*} \otimes \mathcal{O}_{V}(2)^{*}\right)=\mathbb{C}^{6}\right) \\
\Longrightarrow H^{3}\left(X,\left.\mathcal{O}_{V}(2)^{*}\right|_{X}\right) & =\mathbb{C}^{44}
\end{aligned}
$$

and $H^{i}\left(X,\left.\mathcal{O}_{V}(2)^{*}\right|_{X}\right), i=0,1,2$, are trivial. Then one gets $H^{3}\left(X,\left.\mathcal{E}_{3}^{*}\right|_{X}\right)=\mathbb{C}^{83}$ by $(\mathrm{B} .32)$ and (B.33). From (B.31) one finds that

$$
\begin{aligned}
H^{1}\left(V, T^{*} V\right) & =\mathbb{C} \Longrightarrow H^{1}\left(X,\left.T^{*} V\right|_{X}\right)=\mathbb{C}, \\
H^{6}\left(V, \wedge^{3} \mathcal{E}_{3}^{*} \otimes T^{*} V\right) & =\mathbb{C}^{24} \Longrightarrow H^{3}\left(X,\left.T^{*} V\right|_{X}\right)=\mathbb{C}^{24},
\end{aligned}
$$

and $H^{0}\left(X,\left.T^{*} V\right|_{X}\right)$ and $H^{2}\left(X,\left.T^{*} V\right|_{X}\right)$ are trivial. Using these results, the exact sequence (B.17) yields the following exact sequences:

$$
\begin{aligned}
& 0 \longrightarrow H^{0}\left(X, T^{*} X\right) \longrightarrow 0, \\
& 0 \longrightarrow \mathbb{C} \longrightarrow H^{1}\left(X, T^{*} X\right) \longrightarrow 0, \\
& 0 \longrightarrow H^{2}\left(X, T^{*} X\right) \longrightarrow \mathbb{C}^{83} \stackrel{f}{\longrightarrow} \mathbb{C}^{24} \longrightarrow H^{3}\left(X, T^{*} X\right) \longrightarrow 0 .
\end{aligned}
$$


Then one obtains

$$
\begin{array}{ll}
H^{1,0}(X)=H^{0}\left(X, T^{*} X\right)=0, & H^{1,1}(X)=H^{1}\left(X, T^{*} X\right)=\mathbb{C}, \\
H^{1,2}(X)=H^{2}\left(X, T^{*} X\right)=\operatorname{ker}(f)=\mathbb{C}^{59}, & H^{1,3}(X)=H^{3}\left(X, T^{*} X\right)=\operatorname{coker}(f)=0 .
\end{array}
$$

As a result, the Hodge diamond is obtained as

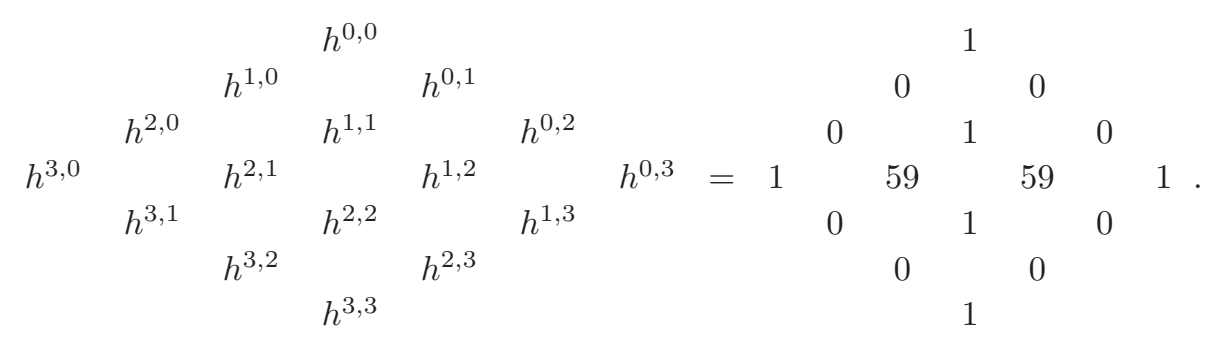

B.2.3 Determinantal Calabi-Yau 3-fold in $(2.25)$ with $\mathcal{F}_{3}=\mathcal{S}^{*}(1) \oplus \mathcal{O}_{V}(1)$

Consider a determinantal Calabi-Yau 3-fold in (2.25) with $\mathcal{F}_{3}=\mathcal{S}^{*}(1) \oplus \mathcal{O}_{V}(1)$. We especially consider a geometric phase, and then the desingularized determinantal CalabiYau 3-fold $X$ is defined by the locus of a holomorphic section of $\mathcal{E}_{3}=\mathcal{E}_{3}^{(2)} \oplus \mathcal{E}_{3}^{(1)}$ on $V^{\prime}=$ $G(2,4) \times \mathbb{P}^{2}$, where $\mathcal{E}_{3}^{(2)}=\mathcal{S}^{*} \otimes \mathcal{O}_{V^{\prime}}(1,0)$ and $\mathcal{E}_{3}^{(1)}=\mathcal{O}_{V^{\prime}}(1,1)$. Using the representations in Example B.4, the Koszul exact sequence (B.3) is given by

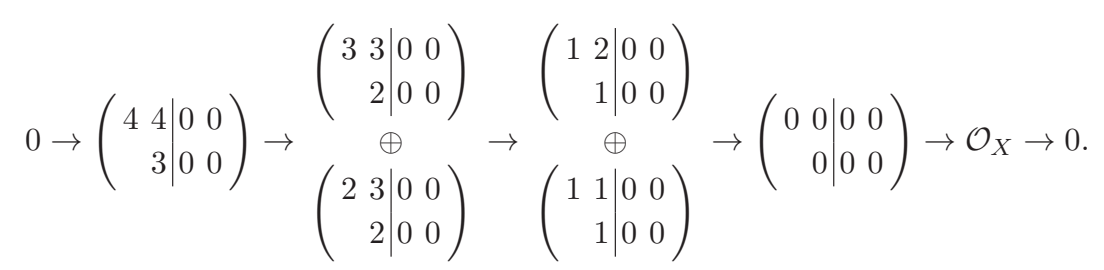

By (B.7) and the Bott-Borel-Weil theorem B.5, one finds that

$$
H^{0}\left(V^{\prime}, \mathcal{O}_{V^{\prime}}\right)=\mathbb{C} \Longrightarrow H^{0,0}(X)=\mathbb{C}, \quad H^{6}\left(V^{\prime}, \wedge^{3} \mathcal{E}_{3}^{*}\right)=\mathbb{C} \Longrightarrow H^{0,3}(X)=\mathbb{C},
$$

and $H^{0,1}(X)$ and $H^{0,2}(X)$ are trivial, i.e. $H^{0,1}(X)=H^{0,2}(X)=0$.

The exact sequence (B.8) gives, for $\mathcal{F}_{V^{\prime}}=\mathcal{E}_{3}^{(2) *}$,

$$
\begin{aligned}
& \left(\begin{array}{rr|rr}
4 & 5 & 0 & 0 \\
3 & 0 & 0
\end{array}\right) \quad\left(\begin{array}{rr|rr}
2 & 4 & 0 & 0 \\
2 & 0 & 0
\end{array}\right) \\
& \oplus \oplus
\end{aligned}
$$

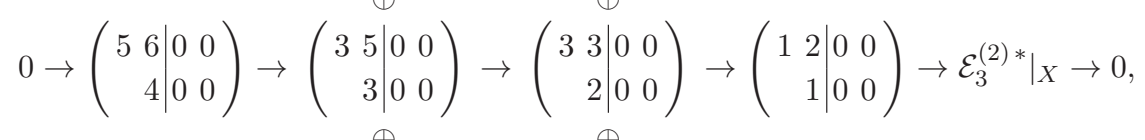

$$
\begin{aligned}
& \left(\begin{array}{rr|rr}
4 & 4 & 0 & 0 \\
3 & 0 & 0
\end{array}\right) \quad\left(\begin{array}{rr|rr}
2 & 3 & 0 & 0 \\
2 & 0 & 0
\end{array}\right)
\end{aligned}
$$

for $\mathcal{F}_{V^{\prime}}=\mathcal{E}_{3}^{(1) *}$,

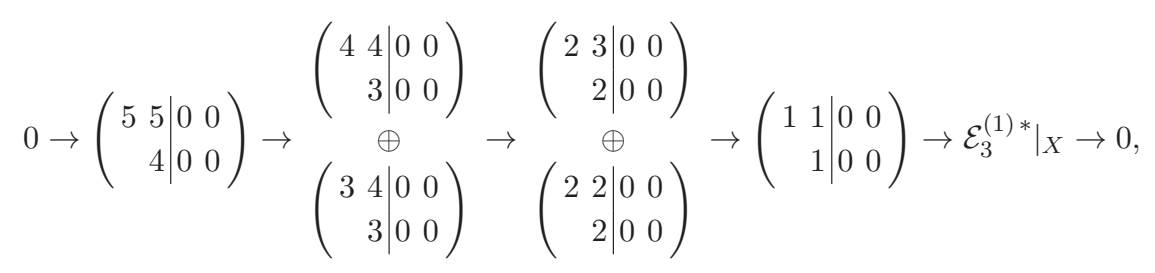


for $\mathcal{F}_{V^{\prime}}=T^{*} G(2,4)$,

$$
\begin{aligned}
& \left(\begin{array}{rr|rr}
3 & 4 & -1 & 0 \\
& 2 & 0 & 0
\end{array}\right) \quad\left(\begin{array}{rr|rr}
1 & 3 & -1 & 0 \\
& 1 & 0 & 0
\end{array}\right) \\
& \left.0 \rightarrow\left(\begin{array}{rr|rr}
4 & 5 & -1 & 0 \\
3 & 0 & 0
\end{array}\right) \rightarrow\left(\begin{array}{rr|rr}
2 & 4 & -1 & 0 \\
2 & 0 & 0
\end{array}\right) \rightarrow\left(\begin{array}{rr|rr}
2 & 2 & -1 & 0 \\
1 & 0 & 0
\end{array}\right) \rightarrow\left(\begin{array}{rr|rr}
0 & 1 & -1 & 0 \\
0 & 0 & 0
\end{array}\right) \rightarrow T^{*} G(2,4)\right|_{X} \rightarrow 0, \\
& \left(\begin{array}{rr|rr}
3 & 3 & -1 & 0 \\
& 2 & 0 & 0
\end{array}\right) \quad\left(\begin{array}{rr|rr}
1 & 2 & -1 & 0 \\
& 1 & 0 & 0
\end{array}\right)
\end{aligned}
$$

and for $\mathcal{F}_{V^{\prime}}=T^{*} \mathbb{P}^{2}$,

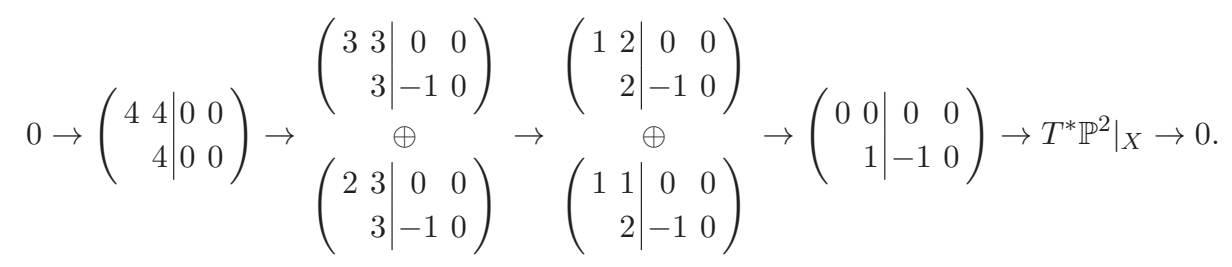

From (B.39) one finds that

$$
\begin{aligned}
\operatorname{ker}\left(d_{0}: H^{6}\left(V^{\prime}, \wedge^{3} \mathcal{E}_{3}^{*} \otimes \mathcal{E}_{3}^{(2) *}\right)\right. & \left.=\mathbb{C}^{60} \longrightarrow H^{6}\left(V^{\prime}, \wedge^{2} \mathcal{E}_{3}^{*} \otimes \mathcal{E}_{3}^{(2) *}\right)=\mathbb{C}^{5}\right) \\
\Longrightarrow H^{3}\left(X,\left.\mathcal{E}_{3}^{(2) *}\right|_{X}\right) & =\mathbb{C}^{55}
\end{aligned}
$$

and $H^{i}\left(X,\left.\mathcal{E}_{3}^{(2) *}\right|_{X}\right), i=0,1,2$, are trivial. From (B.40) one finds that

$$
\begin{aligned}
\operatorname{ker}\left(d_{0}: H^{6}\left(V^{\prime}, \wedge^{3} \mathcal{E}_{3}^{*} \otimes \mathcal{E}_{3}^{(1) *}\right)\right. & \left.=\mathbb{C}^{18} \longrightarrow H^{6}\left(V^{\prime}, \wedge^{2} \mathcal{E}_{3}^{*} \otimes \mathcal{E}_{3}^{(1) *}\right)=\mathbb{C}\right) \\
\Longrightarrow H^{3}\left(X,\left.\mathcal{E}_{3}^{(1) *}\right|_{X}\right) & =\mathbb{C}^{17},
\end{aligned}
$$

and $H^{i}\left(X,\left.\mathcal{E}_{3}^{(1) *}\right|_{X}\right), i=0,1,2$, are trivial. Then one gets $H^{3}\left(X,\left.\mathcal{E}_{3}^{*}\right|_{X}\right)=\mathbb{C}^{72}$ by $(\mathrm{B} .43)$ and (B.44). From (B.41) one finds that

$$
\begin{aligned}
H^{1}\left(V^{\prime}, T^{*} G(2,4)\right) & =\mathbb{C} \Longrightarrow H^{1}\left(X,\left.T^{*} G(2,4)\right|_{X}\right)=\mathbb{C}, \\
H^{6}\left(V^{\prime}, \wedge^{3} \mathcal{E}_{3}^{*} \otimes T^{*} G(2,4)\right) & =\mathbb{C}^{15} \Longrightarrow H^{3}\left(X,\left.T^{*} G(2,4)\right|_{X}\right)=\mathbb{C}^{15},
\end{aligned}
$$

and $H^{0}\left(X,\left.T^{*} G(2,4)\right|_{X}\right)$ and $H^{2}\left(X,\left.T^{*} G(2,4)\right|_{X}\right)$ are trivial. From (B.42) one finds that

$$
\begin{aligned}
H^{1}\left(V^{\prime}, T^{*} \mathbb{P}^{2}\right) & =\mathbb{C} \Longrightarrow H^{1}\left(X,\left.T^{*} \mathbb{P}^{2}\right|_{X}\right)=\mathbb{C}, \\
H^{6}\left(V^{\prime}, \wedge^{3} \mathcal{E}_{3}^{*} \otimes T^{*} \mathbb{P}^{2}\right) & =\mathbb{C}^{8} \Longrightarrow H^{3}\left(X,\left.T^{*} \mathbb{P}^{2}\right|_{X}\right)=\mathbb{C}^{8},
\end{aligned}
$$

and $H^{0}\left(X,\left.T^{*} \mathbb{P}^{2}\right|_{X}\right)$ and $H^{2}\left(X,\left.T^{*} \mathbb{P}^{2}\right|_{X}\right)$ are trivial. Then one gets $H^{1}\left(X,\left.T^{*} V^{\prime}\right|_{X}\right)=\mathbb{C}^{2}$ and $H^{3}\left(X,\left.T^{*} V^{\prime}\right|_{X}\right)=\mathbb{C}^{23}$ by (B.45) and (B.46). Using these results, the exact sequence (B.17) yields the following exact sequences:

$$
\begin{aligned}
& 0 \longrightarrow H^{0}\left(X, T^{*} X\right) \longrightarrow 0, \\
& 0 \longrightarrow \mathbb{C}^{2} \longrightarrow H^{1}\left(X, T^{*} X\right) \longrightarrow 0, \\
& 0 \longrightarrow H^{2}\left(X, T^{*} X\right) \longrightarrow \mathbb{C}^{72} \stackrel{f}{\longrightarrow} \mathbb{C}^{23} \longrightarrow H^{3}\left(X, T^{*} X\right) \longrightarrow 0 .
\end{aligned}
$$


Then one obtains

$$
\begin{array}{ll}
H^{1,0}(X)=H^{0}\left(X, T^{*} X\right)=0, & H^{1,1}(X)=H^{1}\left(X, T^{*} X\right)=\mathbb{C}^{2}, \\
H^{1,2}(X)=H^{2}\left(X, T^{*} X\right)=\operatorname{ker}(f)=\mathbb{C}^{49}, & H^{1,3}(X)=H^{3}\left(X, T^{*} X\right)=\operatorname{coker}(f)=0 .
\end{array}
$$

As a result, the Hodge diamond is obtained as

$$
\begin{array}{cccccccccccccccc} 
& & & h^{0,0} & & & & & & & & & & & \\
& & h^{1,0} & & h^{0,1} & & & & & 0 & & 0 & & \\
h^{3,0} & h^{2,0} & & h^{1,1} & & h^{0,2} & & & 0 & & 2 & & 0 & \\
& h^{2,1} & & h^{1,2} & & h^{0,3}=1 & & 49 & & 49 & & 1 \\
& h^{3,1} & h^{2,2} & & h^{1,3} & & & 0 & & 2 & & 0 & \\
& & h^{3,2} & & h^{2,3} & & & & & 0 & & 0 & & \\
& & & h^{3,3} & & & & & & & 1 & & &
\end{array}
$$

\section{Genus-0 invariants of determinantal Calabi-Yau 4-folds}

Following the techniques introduced in section 4.2, here we consider genus-0 invariants of the desingularized determinantal Calabi-Yau 4-folds clarified in appendix A.2 which are described by $\mathrm{U}(k) \times \mathrm{U}\left(\ell_{p}^{\vee}\right)$ PAX models with $k, \ell_{p}^{\vee}=1,2$, while ignoring the examples with the universal quotient bundle $\mathcal{Q}$ in $\mathcal{F}_{p}$. For the computation, we need to evaluate the classical intersection numbers (4.11) simply denoted by

$$
H \sigma_{1}^{2}, \quad H \sigma_{2}, \quad H \sigma_{1} \tau_{1}, \quad H \tau_{1}^{2}, \quad H \tau_{2}
$$

We also compute their Hodge numbers by analysing the Koszul complex introduced in appendix B. Note that here the genus-0 invariants $n_{d_{1}, d_{2}}(H)$ in (4.7) are denoted as

$$
\begin{array}{ll}
n_{d_{1}, d_{2}, 11}=n_{d_{1}, d_{2}}\left(\sigma_{1}^{2}\right), & n_{d_{1}, d_{2}, \sigma}=n_{d_{1}, d_{2}}\left(\sigma_{2}\right), \quad n_{d_{1}, d_{2}, 12}=n_{d_{1}, d_{2}}\left(\sigma_{1} \tau_{1}\right) \\
n_{d_{1}, d_{2}, 22}=n_{d_{1}, d_{2}}\left(\tau_{1}^{2}\right), & n_{d_{1}, d_{2}, \tau}=n_{d_{1}, d_{2}}\left(\tau_{2}\right)
\end{array}
$$

\section{C.1 Sextic family}

As a higher dimensional analogue of the quintic family discussed in section 4.3.1, we consider the determinantal Calabi-Yau 4-folds in (A.6) which can be described by $\mathrm{U}(1) \times \mathrm{U}(1)$ GLSMs. The "trivial" determinantal Calabi-Yau 4-fold with $\mathcal{F}_{p}=\mathcal{O}_{V}(6)$ on $V=\mathbb{P}^{5}$ corresponds to the sextic Calabi-Yau 4-fold $X_{1}$ with $n=6, r=1, d_{1}=6$ in section 3.2.1. The Hodge numbers are $\left(h^{1,1}, h^{2,1}, h^{2,2}, h^{3,1}\right)=(1,0,1752,426)$. The classical intersection number (3.14) and the genus-0 invariants (3.16) of the sextic $X_{1}$ are given by $\kappa=6$ and

$$
\begin{aligned}
& n_{1,11}=60480, n_{2,11}=440884080, n_{3,11}=6255156277440, \\
& n_{4,11}=117715791990353760, \ldots,
\end{aligned}
$$

respectively [30]. 
Several genus-0 invariants of the sextic family (A.6) are summarized in table 7, where one can check that $\left(h^{1,0}, h^{2,0}\right)=(0,0)$ and there is a relation originated from the extremal transition:

$$
n_{d, 11}=\sum_{d_{2}=0}^{N} n_{d, d_{2}, 11},
$$

where $N$ is a certain finite positive integer.

\begin{tabular}{|c|c|c|c|c|c|c|}
\hline \multicolumn{7}{|c|}{$\mathcal{F}_{p}=\mathcal{O}_{V}(1) \oplus \mathcal{O}_{V}(5):\left(h^{1,1}, h^{2,1}, h^{2,2}, h^{3,1}\right)=(2,0,1452,350),\left(n_{d_{1}, d_{2}, 22}=0\right)$} \\
\hline \multicolumn{3}{|c|}{ Intersection numbers } & \multicolumn{4}{|c|}{$\sigma_{1}^{4}=6, \quad \sigma_{1}^{3} \tau_{1}=5, \quad \sigma_{1}^{2} \tau_{1}^{2}=0, \quad \sigma_{1} \tau_{1}^{3}=0, \quad \tau_{1}^{4}=0$} \\
\hline$n_{d_{1}, d_{2}, 11}$ & $d_{1}=0$ & 1 & 2 & 3 & 4 & 5 \\
\hline$d_{2}=0$ & & 11100 & 5974850 & 5337637100 & 5961261947000 & 7549696778037500 \\
\hline 1 & 0 & 47800 & 139595300 & 341903160900 & 781526104500800 & 1722498037214056500 \\
\hline 2 & 0 & 2300 & 288301400 & 2474705048600 & 12772788325116200 & 51691531760557694400 \\
\hline 3 & 0 & -900 & 10709800 & 3363595465000 & 51229393390313200 & 425107528698920155100 \\
\hline 4 & 0 & 200 & -5618400 & 103567454100 & 51958819718170400 & 1158355364337024993600 \\
\hline 5 & 0 & -20 & 2835300 & -51911590000 & 1403818415592500 & 938149531037521616000 \\
\hline$n_{d_{1}, d_{2}, 12}$ & $d_{1}=0$ & 1 & 2 & 3 & 4 & 5 \\
\hline$d_{2}=0$ & & 2875 & 1218500 & 951619125 & 969870120000 & 1146529444438125 \\
\hline 1 & 25 & 43025 & 80799950 & 156102470525 & 304442819735350 & 596487343049391900 \\
\hline 2 & 0 & 7075 & 268094350 & 1716513933050 & 7342810580729600 & 25898280425210696100 \\
\hline 3 & 0 & -3325 & 27921700 & 3182702667725 & 38694830186103150 & 274863504753902753625 \\
\hline 4 & 0 & 850 & -16827350 & 244126695475 & 49743335407652800 & 920672555667202043750 \\
\hline 5 & 0 & -100 & 9475875 & -139333207500 & 3049558752331250 & 905924121779310315625 \\
\hline \multicolumn{7}{|c|}{$\mathcal{F}_{p}=\mathcal{O}_{V}(2) \oplus \mathcal{O}_{V}(4):\left(h^{1,1}, h^{2,1}, h^{2,2}, h^{3,1}\right)=(2,0,984,233),\left(n_{d_{1}, d_{2}, 22}=0\right)$} \\
\hline \multicolumn{3}{|c|}{ Intersection numbers } & \multicolumn{4}{|c|}{$\sigma_{1}^{4}=6, \quad \sigma_{1}^{3} \tau_{1}=8, \quad \sigma_{1}^{2} \tau_{1}^{2}=0, \quad \sigma_{1} \tau_{1}^{3}=0, \quad \tau_{1}^{4}=0$} \\
\hline$n_{d_{1}, d_{2}, 11}$ & $d_{1}=0$ & 1 & 2 & 3 & 4 & 5 \\
\hline$d_{2}=0$ & & 5152 & 933968 & 274818272 & 100238592192 & 41343866067168 \\
\hline 1 & 0 & 30464 & 30631168 & 24983703040 & 18823860029184 & 13607365845297920 \\
\hline 2 & 0 & 24384 & 148136832 & 344078200064 & 540875866571264 & 689210122091722112 \\
\hline 3 & 0 & 512 & 196648704 & 1484112439552 & 5090782137162496 & 11687877699117056512 \\
\hline 4 & 0 & -32 & 63767008 & 2480102598912 & 20463045985953792 & 88125209117491672192 \\
\hline 5 & 0 & 0 & 833024 & 1597722995712 & 39020950213890816 & 338009904921145655552 \\
\hline$n_{d_{1}, d_{2}, 12}$ & $d_{1}=0$ & 1 & 2 & 3 & 4 & 5 \\
\hline$d_{2}=0$ & & 1280 & 184576 & 46965504 & 15535610112 & 5954616410880 \\
\hline 1 & 64 & 32000 & 19441024 & 12147379712 & 7666954166848 & 4863492485707008 \\
\hline 2 & 0 & 45696 & 155375232 & 264748238336 & 338696685934592 & 370700658349715200 \\
\hline 3 & 0 & 1792 & 288667776 & 1555786807552 & 4265241324428224 & 8297783461923275008 \\
\hline 4 & 0 & -128 & 121767232 & 3295974933504 & 21444381835426304 & 77477798899226917376 \\
\hline 5 & 0 & 0 & 2638208 & 2582769371136 & 49128078374461248 & 354147270418259729152 \\
\hline \multicolumn{7}{|c|}{$\mathcal{F}_{p}=\mathcal{O}_{V}(3)^{\oplus 2}:\left(h^{1,1}, h^{2,1}, h^{2,2}, h^{3,1}\right)=(2,0,780,182),\left(n_{d_{1}, d_{2}, 22}=0\right)$} \\
\hline \multicolumn{3}{|c|}{ Intersection numbers } & \multicolumn{4}{|c|}{$\sigma_{1}^{4}=6, \quad \sigma_{1}^{3} \tau_{1}=9, \quad \sigma_{1}^{2} \tau_{1}^{2}=0, \quad \sigma_{1} \tau_{1}^{3}=0, \quad \tau_{1}^{4}=0$} \\
\hline$n_{d_{1}, d_{2}, 11}$ & $d_{1}=0$ & 1 & 2 & 3 & 4 & 5 \\
\hline$d_{2}=0$ & & 3996 & 528012 & 111620808 & 29176888824 & 8616413173572 \\
\hline 1 & 0 & 26244 & 18834444 & 10994448492 & 5928552658692 & 3066843382569540 \\
\hline 2 & 0 & 26244 & 107617896 & 174298692024 & 193364991313056 & 174792376622296872 \\
\hline 3 & 0 & 3996 & 186923376 & 916489110132 & 2154915386009316 & 3451110870456005940 \\
\hline 4 & 0 & 0 & 107617896 & 2025684267264 & 10761703969222224 & 31389410244093246936 \\
\hline 5 & 0 & 0 & 18834444 & 2025684267264 & 27258432537609648 & 151496100969322358520 \\
\hline
\end{tabular}




\begin{tabular}{|c|c|c|c|c|c|c|}
\hline$n_{d_{1}, d_{2}, 12}$ & $d_{1}=0$ & 1 & 2 & 3 & 4 & 5 \\
\hline$d_{2}=0$ & & 1053 & 105624 & 19272978 & 4557793536 & 1248939462915 \\
\hline 1 & 81 & 27945 & 12158991 & 5429580417 & 2449467003132 & 1110716561847951 \\
\hline 2 & 0 & 50787 & 114975450 & 136362364218 & 122998311431838 & 95426230272412266 \\
\hline 3 & 0 & 10935 & 280385064 & 978583930209 & 1836173061024606 & 2489535822420553203 \\
\hline 4 & 0 & 0 & 207878238 & 2745855802998 & 11484979618590612 & 28070822227226686614 \\
\hline 5 & 0 & 0 & 44344341 & 3331196998794 & 34978559566645782 & 161618974169879654460 \\
\hline \multicolumn{7}{|c|}{$\mathcal{F}_{p}=\mathcal{O}_{V}(2)^{\oplus 3}:\left(h^{1,1}, h^{2,1}, h^{2,2}, h^{3,1}\right)=(2,0,600,137)$} \\
\hline \multicolumn{3}{|c|}{ Intersection numbers } & \multicolumn{4}{|c|}{$\sigma_{1}^{4}=6, \quad \sigma_{1}^{3} \tau_{1}=12, \quad \sigma_{1}^{2} \tau_{1}^{2}=8, \quad \sigma_{1} \tau_{1}^{3}=0, \quad \tau_{1}^{4}=0$} \\
\hline$n_{d_{1}, d_{2}, 11}$ & $d_{1}=0$ & 1 & 2 & 3 & 4 & 5 \\
\hline$d_{2}=0$ & & 1104 & 13464 & 196848 & 3102144 & 52343184 \\
\hline 1 & 0 & 14016 & 1708224 & 120184704 & 6485946432 & 298466405568 \\
\hline 2 & 0 & 30240 & 24792672 & 6198463872 & 882834675456 & 89849127844224 \\
\hline 3 & 0 & 14016 & 107708352 & 89554908096 & 31167666411840 & 6550759785428544 \\
\hline 4 & 0 & 1104 & 172438656 & 529075766016 & 444446786009856 & 187337558146915488 \\
\hline 5 & 0 & 0 & 107708352 & 1473092769024 & 3125024856532800 & 2640802670799084864 \\
\hline$n_{d_{1}, d_{2}, 12}$ & $d_{1}=0$ & 1 & 2 & 3 & 4 & 5 \\
\hline$d_{2}=0$ & & 384 & 3744 & 47232 & 670272 & 10462080 \\
\hline 1 & 96 & 16128 & 1240896 & 68822016 & 3180759840 & 130766711040 \\
\hline 2 & 0 & 60480 & 28308096 & 5320032000 & 629362595328 & 55970237060352 \\
\hline 3 & 0 & 39936 & 168950976 & 102040729344 & 28860224852448 & 5218075942288896 \\
\hline 4 & 0 & 4032 & 344877312 & 753418660608 & 505820047097088 & 181160699096012928 \\
\hline 5 & 0 & 0 & 261882432 & 2521023722496 & 4224146727978144 & 3003266065628143872 \\
\hline$n_{d_{1}, d_{2}, 22}$ & $d_{1}=0$ & 1 & 2 & 3 & 4 & 5 \\
\hline$d_{2}=0$ & & 0 & 0 & 0 & 0 & 0 \\
\hline 1 & 96 & 7296 & 358080 & 14528256 & 528892320 & 17932977792 \\
\hline 2 & 0 & 38592 & 13180992 & 1930140672 & 186374277120 & 13980674976768 \\
\hline 3 & 0 & 31104 & 97081920 & 47789472384 & 11376521292384 & 1772136040869504 \\
\hline 4 & 0 & 3648 & 226720032 & 411451649280 & 237591482788608 & 74578392862419072 \\
\hline 5 & 0 & 0 & 190013376 & 1533694993920 & 2239140736880928 & 1413152060075199360 \\
\hline \multicolumn{7}{|c|}{$\mathcal{F}_{p}=\mathcal{O}_{V}(1) \oplus \mathcal{O}_{V}(2) \oplus \mathcal{O}_{V}(3):\left(h^{1,1}, h^{2,1}, h^{2,2}, h^{3,1}\right)=(2,0,732,170)$} \\
\hline \multicolumn{3}{|c|}{ Intersection numbers } & \multicolumn{4}{|c|}{$\sigma_{1}^{4}=6, \quad \sigma_{1}^{3} \tau_{1}=11, \quad \sigma_{1}^{2} \tau_{1}^{2}=6, \quad \sigma_{1} \tau_{1}^{3}=0, \quad \tau_{1}^{4}=0$} \\
\hline$n_{d_{1}, d_{2}, 11}$ & $d_{1}=0$ & 1 & 2 & 3 & 4 & 5 \\
\hline$d_{2}=0$ & & 1547 & 29197 & 664966 & 16655276 & 449773471 \\
\hline 1 & 0 & 17415 & 3387141 & 390771675 & 34733397231 & 2631133285191 \\
\hline 2 & 0 & 31234 & 40960891 & 16736425757 & 3941526901738 & 666261117547152 \\
\hline 3 & 0 & 10138 & 143247860 & 196780670876 & 113318694822063 & 39594013933183682 \\
\hline 4 & 0 & 147 & 172936880 & 924949591952 & 1299468913703502 & 913025985733713523 \\
\hline 5 & 0 & -1 & 72200686 & 1974502966996 & 7224038700165558 & 10275054838460143332 \\
\hline$n_{d_{1}, d_{2}, 12}$ & $d_{1}=0$ & 1 & 2 & 3 & 4 & 5 \\
\hline$d_{2}=0$ & & 546 & 8022 & 159708 & 3637752 & 91579530 \\
\hline 1 & 85 & 19550 & 2414660 & 220345310 & 16823027671 & 1141797701894 \\
\hline 2 & 0 & 61679 & 45928018 & 14111157767 & 2763493715428 & 408754388583466 \\
\hline 3 & 0 & 28503 & 221344804 & 220417729691 & 103132562736885 & 31012895322615811 \\
\hline 4 & 0 & 607 & 341620521 & 1297053558298 & 1454510648416542 & 868094813403547075 \\
\hline 5 & 0 & -5 & 173699427 & 3333597269546 & 9614835008030966 & 11495407244589530600 \\
\hline$n_{d_{1}, d_{2}, 22}$ & $d_{1}=0$ & 1 & 2 & 3 & 4 & 5 \\
\hline$d_{2}=0$ & & 0 & 0 & 0 & 0 & 0 \\
\hline 1 & 66 & 7944 & 665916 & 45550248 & 2770233906 & 155976830808 \\
\hline
\end{tabular}




\begin{tabular}{|c|c|c|c|c|c|c|}
\hline 2 & 0 & 33774 & 19165296 & 4739973306 & 774617782512 & 98109047877348 \\
\hline 3 & 0 & 18270 & 110465976 & 92373759582 & 37217080568574 & 9812878163478678 \\
\hline 4 & 0 & 498 & 190515834 & 619593755556 & 610866205882020 & 325210132883359866 \\
\hline 5 & 0 & -6 & 104930694 & 1742691953364 & 4476709752274068 & 4833888549187725312 \\
\hline \multicolumn{7}{|c|}{$\mathcal{F}_{p}=\mathcal{O}_{V}(1)^{\oplus 2} \oplus \mathcal{O}_{V}(4):\left(h^{1,1}, h^{2,1}, h^{2,2}, h^{3,1}\right)=(2,0,1068,254)$} \\
\hline \multicolumn{3}{|c|}{ Intersection numbers } & \multicolumn{4}{|c|}{$\sigma_{1}^{4}=6, \quad \sigma_{1}^{3} \tau_{1}=9, \quad \sigma_{1}^{2} \tau_{1}^{2}=4, \quad \sigma_{1} \tau_{1}^{3}=0, \quad \tau_{1}^{4}=0$} \\
\hline$n_{d_{1}, d_{2}, 11}$ & $d_{1}=0$ & 1 & 2 & 3 & 4 & 5 \\
\hline$d_{2}=0$ & & 2796 & 111420 & 5415876 & 297906744 & 17836490652 \\
\hline 1 & 0 & 25368 & 11074512 & 2923919928 & 597405875232 & 103952142270864 \\
\hline 2 & 0 & 31452 & 96756426 & 92450088216 & 50890408972608 & 20094224706004404 \\
\hline 3 & 0 & 1020 & 215383416 & 746257722660 & 1032988712010672 & 857190260480341476 \\
\hline 4 & 0 & -168 & 115603488 & 2184801219792 & 7902484863731640 & 13590303106004642712 \\
\hline 5 & 0 & 12 & 2269284 & 2399750411904 & 27079947685057788 & 100137122291641121868 \\
\hline$n_{d_{1}, d_{2}, 12}$ & $d_{1}=0$ & 1 & 2 & 3 & 4 & 5 \\
\hline$d_{2}=0$ & & 960 & 30096 & 1297728 & 65879904 & 3714521280 \\
\hline 1 & 57 & 27438 & 7644177 & 1605192702 & 282936273240 & 44282085901044 \\
\hline 2 & 0 & 3657 & 104754642 & 75519972306 & 34661964976188 & 12004979836835943 \\
\hline 3 & 0 & -681 & 320554332 & 807765491547 & 910677818722380 & 651630952247594127 \\
\hline 4 & 0 & 57 & 222052140 & 2956519017480 & 8551880283800160 & 12515007949041304332 \\
\hline 5 & 0 & 0 & 7374009 & 3919508517492 & 34811597561726766 & 108347819585126752047 \\
\hline$n_{d_{1}, d_{2}, 22}$ & $d_{1}=0$ & 1 & 2 & 3 & 4 & 5 \\
\hline$d_{2}=0$ & & 0 & 0 & 0 & 0 & 0 \\
\hline 1 & 36 & 10104 & 2000580 & 323373624 & 46020698976 & 6018914291280 \\
\hline 2 & 0 & 28260 & 39402408 & 23483405064 & 9172938062064 & 2759901496567260 \\
\hline 3 & 0 & 2340 & 140674032 & 304708034988 & 301404502100016 & 191986430652018684 \\
\hline 4 & 0 & -420 & 108023472 & 1249191338400 & 3231021483376128 & 4278942916449123696 \\
\hline 5 & 0 & 36 & 4447332 & 1793863512336 & 14384155887545592 & 40973480285079165564 \\
\hline \multicolumn{7}{|c|}{$\mathcal{F}_{p}=\mathcal{O}_{V}(1)^{\oplus 2} \oplus \mathcal{O}_{V}(2)^{\oplus 2}:\left(h^{1,1}, h^{2,1}, h^{2,2}, h^{3,1}\right)=(2,0,588,134)$} \\
\hline \multicolumn{3}{|c|}{ Intersection numbers } & \multicolumn{4}{|c|}{$\sigma_{1}^{4}=6, \quad \sigma_{1}^{3} \tau_{1}=13, \quad \sigma_{1}^{2} \tau_{1}^{2}=12, \quad \sigma_{1} \tau_{1}^{3}=4, \quad \tau_{1}^{4}=0$} \\
\hline$n_{d_{1}, d_{2}, 11}$ & $d_{1}=0$ & 1 & 2 & 3 & 4 & 5 \\
\hline$d_{2}=0$ & & 624 & 1512 & 2448 & 2352 & 3696 \\
\hline 1 & 0 & 10552 & 560168 & 12419664 & 167237208 & 1611465704 \\
\hline 2 & 0 & 29420 & 12216604 & 1270882648 & 63182183032 & 1933845747256 \\
\hline 3 & 0 & 17892 & 74261296 & 28590861496 & 4065328815272 & 310191664249228 \\
\hline 4 & 0 & 1988 & 162756004 & 245615224064 & 91643735242360 & 15636111812478864 \\
\hline 5 & 0 & 4 & 139994444 & 965140012064 & 959192743343064 & 351280339787531780 \\
\hline$n_{d_{1}, d_{2}, 12}$ & $d_{1}=0$ & 1 & 2 & 3 & 4 & 5 \\
\hline$d_{2}=0$ & & 256 & 504 & 768 & 784 & 1280 \\
\hline 1 & 97 & 12412 & 431558 & 7712632 & 90771993 & 798269252 \\
\hline 2 & 0 & 59597 & 14304660 & 1139364456 & 47795706040 & 1296194947220 \\
\hline 3 & 0 & 51377 & 118235744 & 33425512482 & 3910275895911 & 259643765174029 \\
\hline 4 & 0 & 7379 & 329186320 & 355897081724 & 107040862884600 & 15662234678584878 \\
\hline 5 & 0 & 19 & 343523755 & 1673793068984 & 1321513374907908 & 410052719920052585 \\
\hline$n_{d_{1}, d_{2}, 22}$ & $d_{1}=0$ & 1 & 2 & 3 & 4 & 5 \\
\hline$d_{2}=0$ & & 0 & 0 & 0 & 0 & 0 \\
\hline 1 & 132 & 7696 & 167672 & 2165664 & 19882116 & 143012720 \\
\hline 2 & 0 & 51428 & 8977216 & 553197376 & 18798577216 & 427186381936 \\
\hline 3 & 0 & 53076 & 91577920 & 21040910824 & 2061819081980 & 117330402886948 \\
\hline
\end{tabular}




\begin{tabular}{|c|c|c|c|c|c|c|}
\hline 4 & 0 & 8732 & 290650960 & 261518705648 & 67487950758592 & 8623613017337400 \\
\hline 5 & 0 & 28 & 332988380 & 1368920496992 & 941519514353136 & 258784559873434676 \\
\hline \multicolumn{7}{|c|}{$\overline{\mathcal{F}_{p}=\mathcal{O}_{V}(1)^{\oplus 3} \oplus \mathcal{O}_{V}(3):\left(h^{1,1}, h^{2,1}, h^{2,2}, h^{3,1}\right)=(2,0,744,173)}$} \\
\hline \multicolumn{3}{|c|}{ Intersection numbers } & \multicolumn{4}{|c|}{$\sigma_{1}^{4}=6, \quad \sigma_{1}^{3} \tau_{1}=12, \quad \sigma_{1}^{2} \tau_{1}^{2}=10, \quad \sigma_{1} \tau_{1}^{3}=3, \quad \tau_{1}^{4}=0$} \\
\hline$n_{d_{1}, d_{2}, 11}$ & $d_{1}=0$ & 1 & 2 & 3 & 4 & 5 \\
\hline$d_{2}=0$ & & 876 & 2754 & 2340 & 4506 & 6384 \\
\hline 1 & 0 & 13224 & 1111680 & 40000284 & 854694672 & 12548849748 \\
\hline 2 & 0 & 31692 & 20814618 & 3502913724 & 285343689342 & 14296324624164 \\
\hline 3 & 0 & 14388 & 105558792 & 66167398224 & 15392391723972 & 1933287656228904 \\
\hline 4 & 0 & 312 & 182631774 & 469032117948 & 288248187932658 & 80990413510330656 \\
\hline 5 & 0 & -12 & 111190416 & 1474197621264 & 2474334577233804 & 1501801964420459808 \\
\hline$n_{d_{1}, d_{2}, 12}$ & $d_{1}=0$ & 1 & 2 & 3 & 4 & 5 \\
\hline$d_{2}=0$ & & 363 & 726 & 702 & 1452 & 1815 \\
\hline 1 & 84 & 15267 & 846654 & 24454455 & 451560702 & 5971621263 \\
\hline 2 & 0 & 63390 & 24022410 & 3100366203 & 213030667314 & 9432564106950 \\
\hline 3 & 0 & 40614 & 165803226 & 76310133777 & 14614556335185 & 1597429012014378 \\
\hline 4 & 0 & 1383 & 364788126 & 670587309870 & 332222425569714 & 80079498824062290 \\
\hline 5 & 0 & -57 & 269640918 & 2524188530034 & 3364141372570932 & 1730133151583505285 \\
\hline$n_{d_{1}, d_{2}, 22}$ & $d_{1}=0$ & 1 & 2 & 3 & 4 & 5 \\
\hline$d_{2}=0$ & & 0 & 0 & 0 & 0 & 0 \\
\hline 1 & 102 & 8976 & 325302 & 6898368 & 99988848 & 1084024620 \\
\hline 2 & 0 & 51372 & 14396598 & 1462589520 & 82497883614 & 3089416732524 \\
\hline 3 & 0 & 38808 & 121109886 & 45893323332 & 7447857934212 & 704572082465418 \\
\hline 4 & 0 & 1716 & 301039440 & 466225271184 & 200138738462880 & 42499979609169498 \\
\hline 5 & 0 & -72 & 242665410 & 1939341503028 & 2272289621718792 & 1043316736192230672 \\
\hline \multicolumn{7}{|c|}{$\mathcal{F}_{p}=\mathcal{O}_{V}(1)^{\oplus 4} \oplus \mathcal{O}_{V}(2):\left(h^{1,1}, h^{2,1}, h^{2,2}, h^{3,1}\right)=(2,0,552,125)$} \\
\hline \multicolumn{3}{|c|}{ Intersection numbers } & \multicolumn{4}{|c|}{$\sigma_{1}^{4}=6, \quad \sigma_{1}^{3} \tau_{1}=14, \quad \sigma_{1}^{2} \tau_{1}^{2}=16, \quad \sigma_{1} \tau_{1}^{3}=9, \quad \tau_{1}^{4}=2$} \\
\hline$n_{d_{1}, d_{2}, 11}$ & $d_{1}=0$ & 1 & 2 & 3 & 4 & 5 \\
\hline$d_{2}=0$ & & 360 & 110 & 0 & 0 & 0 \\
\hline 1 & 0 & 7780 & 181660 & 1182060 & 3226100 & 4267500 \\
\hline 2 & 0 & 27260 & 5703230 & 247915040 & 4199645570 & 35730530600 \\
\hline 3 & 0 & 21540 & 47022520 & 8368536780 & 487011519380 & 13321298891500 \\
\hline 4 & 0 & 3500 & 137972490 & 101219111200 & 16761773920870 & 1158268337627600 \\
\hline 5 & 0 & 40 & 161154360 & 546681379380 & 252762644451260 & 40097079604396400 \\
\hline$n_{d_{1}, d_{2}, 12}$ & $d_{1}=0$ & 1 & 2 & 3 & 4 & 5 \\
\hline$d_{2}=0$ & & 165 & 40 & 0 & 0 & 0 \\
\hline 1 & 100 & 9345 & 147740 & 785690 & 1894775 & 2320375 \\
\hline 2 & 0 & 55990 & 6844020 & 231492010 & 3350412730 & 25503215825 \\
\hline 3 & 0 & 62310 & 76006130 & 10030946845 & 485539930370 & 11668962937750 \\
\hline 4 & 0 & 13125 & 282277260 & 149233093550 & 20076931277380 & 1199570758766725 \\
\hline 5 & 0 & 185 & 399094290 & 960929261945 & 354879437832140 & 48004138643512850 \\
\hline$n_{d_{1}, d_{2}, 22}$ & $d_{1}=0$ & 1 & 2 & 3 & 4 & 5 \\
\hline$d_{2}=0$ & & 0 & 0 & 0 & 0 & 0 \\
\hline 1 & 170 & 6860 & 65700 & 250120 & 468350 & 468350 \\
\hline 2 & 0 & 58020 & 5042710 & 129717540 & 1502417400 & 9512031410 \\
\hline 3 & 0 & 77400 & 70019970 & 7403781260 & 296605169360 & 6050035785700 \\
\hline 4 & 0 & 18700 & 298541880 & 129860240700 & 14833108148590 & 766859594261640 \\
\hline 5 & 0 & 300 & 465232050 & 936876897400 & 298655994540650 & 35488204982996150 \\
\hline \multicolumn{7}{|c|}{$\mathcal{F}_{p}=\mathcal{O}_{V}(1)^{\oplus 6}:\left(h^{1,1}, h^{2,1}, h^{2,2}, h^{3,1}\right)=(2,0,492,110),\left(n_{d_{1}, d_{2}, 22}=n_{d_{2}, d_{1}, 11}\right)[11]$} \\
\hline
\end{tabular}




\begin{tabular}{|c|c|c|c|c|c|c|}
\hline \multicolumn{3}{|c|}{ Intersection numbers } & \multirow{2}{*}{\multicolumn{2}{|c|}{$\begin{array}{cc}\sigma_{1}^{4}=6, & \sigma_{1}^{3} \tau_{1}=15 \\
2 & 3\end{array}$}} & \multirow{2}{*}{$\begin{array}{cc}\sigma_{1}^{2} \tau_{1}^{2}=20, & \sigma_{1} \tau_{1}^{3}=15 \\
4\end{array}$} & \multirow[t]{2}{*}{$\tau_{1}^{4}=6$} \\
\hline$n_{d_{1}, d_{2}, 11}$ & $d_{1}=0$ & 1 & & & & \\
\hline$d_{2}=0$ & & 210 & 0 & 0 & 0 & 0 \\
\hline 1 & 0 & 5670 & 59430 & 100170 & 34650 & 1680 \\
\hline 2 & 0 & 24360 & 2579640 & 47382930 & 264433680 & 546221760 \\
\hline 3 & 0 & 24360 & 28015260 & 2324403900 & 55841697870 & 539959428960 \\
\hline 4 & 0 & 5670 & 107096220 & 38404166850 & 2848564316640 & 80315543697900 \\
\hline 5 & 0 & 210 & 165382980 & 277070715810 & 60035324018880 & 4163431890254700 \\
\hline$n_{d_{1}, d_{2}, 12}$ & $d_{1}=0$ & 1 & 2 & 3 & 4 & 5 \\
\hline$d_{2}=0$ & & 105 & 0 & 0 & 0 & 0 \\
\hline 1 & 105 & 6930 & 50715 & 71085 & 21420 & 945 \\
\hline 2 & 0 & 50715 & 3166800 & 45928155 & 221593050 & 413457450 \\
\hline 3 & 0 & 71085 & 45928155 & 2851172100 & 57546197940 & 493317415605 \\
\hline 4 & 0 & 21420 & 221593050 & 57546197940 & 3492450469200 & 85788539294850 \\
\hline 5 & 0 & 945 & 413457450 & 493317415605 & 85788539294850 & 5102793274479600 \\
\hline
\end{tabular}

Table 7. Genus-0 invariants of determinantal 4-folds in (A.6) with $V=\mathbb{P}^{5}$.

\section{C.2 Determinantal Calabi-Yau 4-folds in (A.8)}

Finally, we consider the determinantal Calabi-Yau 4-folds with $p \neq 2$ in (A.8) which are described by $\mathrm{U}(2) \times \mathrm{U}(2)$ GLSMs, while ignoring the examples with the universal quotient bundle $\mathcal{Q}$ in $\mathcal{F}_{p}$. We summarized the genus-0 invariants of (C.2) in table 8, where one can check that $\left(h^{1,0}, h^{2,0}\right)=(0,0)$. For the Calabi-Yau 4-folds with $\mathcal{F}_{p}=\mathcal{S}^{*} \oplus \mathcal{O}_{V}(1)^{\oplus 2}$, $\left(\mathcal{S}^{*}\right)^{\oplus 2} \oplus \mathcal{O}_{V}(1),\left(\mathcal{S}^{*}\right)^{\oplus 3}$, just due to a technical complexity, instead of the Hodge numbers we give the $\chi_{y}$-genera $\chi_{i}=\sum_{j=0}^{4}(-1)^{j} h^{i, j}, i=0,1,2$ obtained by the formula (B.18).

Note that the determinantal 4 -fold with $\mathcal{F}_{p}=\operatorname{Sym}^{2} \mathcal{S}^{*}$ in (A.8) is an irreducible holomorphic symplectic variety with $\left(h^{1,0}, h^{2,0}\right)=(0,1)$, and all the genus-0 invariants vanished. This property is known to be a general phenomenon for irreducible holomorphic symplectic varieties (see e.g. [62]).

\begin{tabular}{|c|rrrrrrrr|}
\hline \multicolumn{7}{|c|}{$\mathcal{F}_{p}=\mathcal{O}_{V}(1)^{\oplus 3}:\left(h^{1,1}, h^{2,1}, h^{2,2}, h^{3,1}\right)=(2,0,384,83),\left(n_{d_{1}, d_{2}, \tau}=0\right)$} \\
\hline \multicolumn{6}{|c}{ Intersection numbers } & \multicolumn{6}{c|}{$\sigma_{1}^{4}=84}$, & $\sigma_{1}^{2} \sigma_{2}=54$, & $\sigma_{1}^{3} \tau_{1}=42$, & $\sigma_{1}^{2} \tau_{1}^{2}=14$, & $\sigma_{1}^{2} \tau_{2}=0$, & $\sigma_{2}^{2}=36$, \\
\hline \multicolumn{2}{|c}{$\sigma_{1} \sigma_{2} \tau_{1}=27}$, & $\sigma_{2} \tau_{1}^{2}=9$, & $\sigma_{2} \tau_{2}=0$, & $\sigma_{1} \tau_{1}^{3}=0$, & $\sigma_{1} \tau_{1} \tau_{2}=0$, & $\tau_{1}^{4}=0$, & $\tau_{1}^{2} \tau_{2}=0$, & $\tau_{2}^{2}=0$ \\
\hline$n_{d_{1}, d_{2}, 11}$ & $d_{1}=0$ & 1 & 2 & 3 & 4 & 5 & 6 \\
\hline$d_{2}=0$ & & 966 & 6258 & 40194 & 313992 & 2465694 & 20471724 \\
1 & 0 & 966 & 79464 & 2850624 & 73342920 & 1577557254 & 30264388560 \\
2 & 0 & 0 & 6258 & 2850624 & 353216472 & 23351152860 & 1075419836442 \\
3 & 0 & 0 & 0 & 40194 & 73342920 & 23351152860 & 3280923722160 \\
4 & 0 & 0 & 0 & 0 & 313992 & 1577557254 & 1075419836442 \\
5 & 0 & 0 & 0 & 0 & 0 & 2465694 & 30264388560 \\
\hline$n_{d_{1}, d_{2}, \sigma}$ & $d_{1}=0$ & 1 & 2 & 3 & 4 & & 5 \\
\hline$d_{2}=0$ & & 639 & 3987 & 25857 & 201888 & 1584999 & 13160502 \\
1 & 0 & 639 & 51804 & 1846260 & 47378196 & 1017817191 & 19510365672 \\
2 & 0 & 0 & 3987 & 1846260 & 229064418 & 15125263182 & 695710713879 \\
3 & 0 & 0 & 0 & 25857 & 47378196 & 15125263182 & 2125753214616 \\
4 & 0 & 0 & 0 & 0 & 201888 & 1017817191 & 695710713879 \\
5 & 0 & 0 & 0 & 0 & 0 & 1584999 & 19510365672 \\
\hline
\end{tabular}




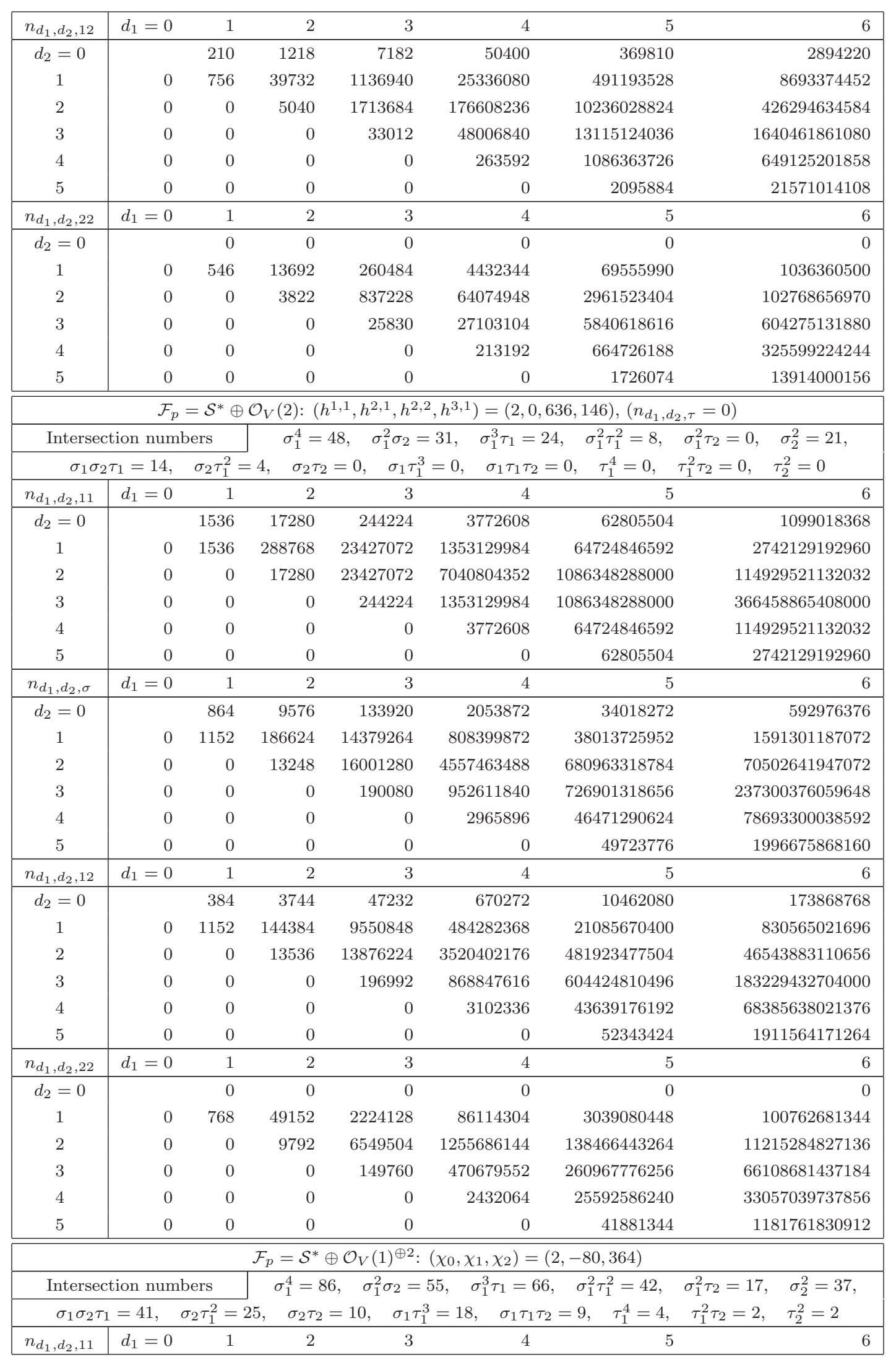




\begin{tabular}{|c|c|c|c|c|c|c|c|}
\hline$d_{2}=0$ & & 496 & 244 & 0 & 0 & 0 & 0 \\
\hline 1 & 0 & 1312 & 38880 & 238740 & 605136 & 785832 & 546816 \\
\hline 2 & 0 & 44 & 41500 & 3158040 & 65572008 & 586267944 & 2862291270 \\
\hline 3 & 0 & 0 & 760 & 1641368 & 253291560 & 11296776072 & 221197911448 \\
\hline 4 & 0 & 0 & 0 & 23284 & 88370324 & 21272865380 & 1596400086708 \\
\hline 5 & 0 & 0 & 0 & -592 & 863776 & 5440233652 & 1834451480648 \\
\hline$n_{d_{1}, d_{2}, \sigma}$ & $d_{1}=0$ & 1 & 2 & 3 & 4 & 5 & 6 \\
\hline$d_{2}=0$ & & 292 & 140 & 0 & 0 & 0 & 0 \\
\hline 1 & 0 & 880 & 24204 & 144470 & 361496 & 466204 & 323180 \\
\hline 2 & 0 & 34 & 27450 & 2002612 & 40636882 & 358061288 & 1731003173 \\
\hline 3 & 0 & 0 & 556 & 1087240 & 162344808 & 7095866952 & 137011548860 \\
\hline 4 & 0 & 0 & 0 & 16830 & 58584756 & 13723707354 & 1011627956132 \\
\hline 5 & 0 & 0 & 0 & -440 & 622184 & 3609161614 & 1188798184500 \\
\hline$n_{d_{1}, d_{2}, 12}$ & $d_{1}=0$ & 1 & 2 & 3 & 4 & 5 & 6 \\
\hline$d_{2}=0$ & & 168 & 72 & 0 & 0 & 0 & 0 \\
\hline 1 & 0 & 1176 & 23688 & 119628 & 271752 & 328080 & 216216 \\
\hline 2 & 0 & 72 & 37524 & 2229240 & 39561432 & 317016480 & 1428444174 \\
\hline 3 & 0 & 0 & 1056 & 1492992 & 191488464 & 7501134312 & 133180541856 \\
\hline 4 & 0 & 0 & 0 & 31128 & 80807544 & 16742371056 & 1125010922424 \\
\hline 5 & 0 & 0 & 0 & -864 & 1140240 & 4994099532 & 1482866868960 \\
\hline$n_{d_{1}, d_{2}, 22}$ & $d_{1}=0$ & 1 & 2 & 3 & 4 & 5 & 6 \\
\hline$d_{2}=0$ & & 0 & 0 & 0 & 0 & 0 & 0 \\
\hline 1 & 0 & 1040 & 10516 & 37316 & 66648 & 66648 & 37316 \\
\hline 2 & 0 & 112 & 31924 & 1296048 & 17835110 & 117630928 & 452438518 \\
\hline 3 & 0 & 0 & 1356 & 1271728 & 125327752 & 4009228688 & 60469656020 \\
\hline 4 & 0 & 0 & 0 & 37768 & 69214312 & 11674274648 & 663812607488 \\
\hline 5 & 0 & 0 & 0 & -1184 & 1367852 & 4298534956 & 1076178498476 \\
\hline$n_{d_{1}, d_{2}, \tau}$ & $d_{1}=0$ & 1 & 2 & 3 & 4 & 5 & 6 \\
\hline$d_{2}=0$ & & 0 & 0 & 0 & 0 & 0 & 0 \\
\hline 1 & 0 & 320 & 4618 & 18418 & 34404 & 34404 & 18418 \\
\hline 2 & 0 & 28 & 10858 & 522792 & 7879519 & 55031576 & 219730439 \\
\hline 3 & 0 & 0 & 382 & 432616 & 47989060 & 1663887680 & 26568226946 \\
\hline 4 & 0 & 0 & 0 & 11040 & 23512884 & 4349537480 & 265076269952 \\
\hline 5 & 0 & 0 & 0 & -320 & 402214 & 1457274582 & 393989709022 \\
\hline \multicolumn{8}{|c|}{$\mathcal{F}_{p}=\left(\mathcal{S}^{*}\right)^{\oplus 2} \oplus \mathcal{O}_{V}(1):\left(\chi_{0}, \chi_{1}, \chi_{2}\right)=(2,-62,292)$} \\
\hline \multicolumn{3}{|c|}{ Intersection numbers } & \multirow{2}{*}{\multicolumn{4}{|c|}{$\begin{array}{c}\sigma_{1}^{4}=92, \quad \sigma_{1}^{2} \sigma_{2}=58, \quad \sigma_{1}^{3} \tau_{1}=92, \quad \sigma_{1}^{2} \tau_{1}^{2}=80 \\
\sigma_{2} \tau_{2}=22, \quad \sigma_{1} \tau_{1}^{3}=56, \quad \sigma_{1} \tau_{1} \tau_{2}=32, \quad \tau_{1}^{4}=32\end{array}$}} & $19, \quad \sigma_{2}^{2}=40$ \\
\hline \multicolumn{3}{|c|}{$\sigma_{1} \sigma_{2} \tau_{1}=56, \quad \sigma_{2} \tau_{1}^{2}=46$} & & & & & $19, \quad \tau_{2}^{2}=13$ \\
\hline$n_{d_{1}, d_{2}, 11}$ & $d_{1}=0$ & 1 & 2 & 3 & 4 & 5 & 6 \\
\hline$d_{2}=0$ & & 180 & 0 & 0 & 0 & 0 & 0 \\
\hline 1 & 0 & 1280 & 11520 & 0 & 0 & 0 & 0 \\
\hline 2 & 0 & 180 & 39420 & 725760 & 1285920 & 110180 & -36660 \\
\hline 3 & 0 & 0 & 11520 & 1981440 & 54604800 & 288737280 & 294952960 \\
\hline 4 & 0 & 0 & 0 & 725760 & 127668480 & 4632572700 & 44638440480 \\
\hline 5 & 0 & 0 & 0 & 0 & 54604800 & 9651020800 & 425483704320 \\
\hline$n_{d_{1}, d_{2}, \sigma}$ & $d_{1}=0$ & 1 & 2 & 3 & 4 & 5 & 6 \\
\hline$d_{2}=0$ & & 90 & 0 & 0 & 0 & 0 & 0 \\
\hline 1 & 0 & 800 & 6560 & 0 & 0 & 0 & 0 \\
\hline 2 & 0 & 130 & 24730 & 428000 & 729020 & 59850 & -19710 \\
\hline 3 & 0 & 0 & 7840 & 1244480 & 32729280 & 167506560 & 166957440 \\
\hline
\end{tabular}




\begin{tabular}{|c|c|c|c|c|c|c|c|}
\hline 4 & 0 & 0 & 0 & 482400 & 80204960 & 2803401590 & 26273744220 \\
\hline 5 & 0 & 0 & 0 & 0 & 35821760 & 6063510400 & 259122088640 \\
\hline$n_{d_{1}, d_{2}, 12}$ & $d_{1}=0$ & 1 & 2 & 3 & 4 & 5 & 6 \\
\hline$d_{2}=0$ & & 60 & 0 & 0 & 0 & 0 & 0 \\
\hline 1 & 0 & 1280 & 7680 & 0 & 0 & 0 & 0 \\
\hline 2 & 0 & 300 & 39420 & 562560 & 846120 & 58940 & -18660 \\
\hline 3 & 0 & 0 & 15360 & 1981440 & 45327360 & 209679360 & 192616960 \\
\hline 4 & 0 & 0 & 0 & 888960 & 127668480 & 4000335300 & 34440454440 \\
\hline 5 & 0 & 0 & 0 & 0 & 63882240 & 9651020800 & 376969835520 \\
\hline$n_{d_{1}, d_{2}, 22}$ & $d_{1}=0$ & 1 & 2 & 3 & 4 & 5 & 6 \\
\hline$d_{2}=0$ & & 0 & 0 & 0 & 0 & 0 & 0 \\
\hline 1 & 0 & 1280 & 3840 & 0 & 0 & 0 & 0 \\
\hline 2 & 0 & 480 & 37200 & 360960 & 439440 & 19040 & -5520 \\
\hline 3 & 0 & 0 & 19200 & 1835520 & 32509440 & 125291520 & 98252800 \\
\hline 4 & 0 & 0 & 0 & 1013760 & 117776640 & 3046100640 & 22457270160 \\
\hline 5 & 0 & 0 & 0 & 0 & 69619200 & 8892083200 & 297725928960 \\
\hline$n_{d_{1}, d_{2}, \tau}$ & $d_{1}=0$ & 1 & 2 & 3 & 4 & 5 & 6 \\
\hline$d_{2}=0$ & & 0 & 0 & 0 & 0 & 0 & 0 \\
\hline 1 & 0 & 560 & 2480 & 0 & 0 & 0 & 0 \\
\hline 2 & 0 & 160 & 17200 & 207920 & 270080 & 14280 & -4140 \\
\hline 3 & 0 & 0 & 7600 & 863840 & 17630880 & 73506240 & 60984000 \\
\hline 4 & 0 & 0 & 0 & 425520 & 55648880 & 1598550680 & 12693468720 \\
\hline 5 & 0 & 0 & 0 & 0 & 30000800 & 4206510400 & 153166332320 \\
\hline \multicolumn{8}{|c|}{$\mathcal{F}_{p}=\left(\mathcal{S}^{*}\right)^{\oplus 3}:\left(\chi_{0}, \chi_{1}, \chi_{2}\right)=(2,-22,132),\left(n_{d_{1}, d_{2}, 22}=n_{d_{2}, d_{1}, 11}, n_{d_{1}, d_{2}, \tau}=n_{d_{2}, d_{1}, \sigma}\right)$} \\
\hline \multicolumn{3}{|c|}{ Intersection numbers } & \multirow{2}{*}{\multicolumn{5}{|c|}{$\begin{array}{lcccc}\sigma_{1}^{4}=102, & \sigma_{1}^{2} \sigma_{2}=63, \quad \sigma_{1}^{3} \tau_{1}=120, & \sigma_{1}^{2} \tau_{1}^{2}=128, & \sigma_{1}^{2} \tau_{2}=72, & \sigma_{2}^{2}=45 \\
\sigma_{2} \tau_{2}=36, & \sigma_{1} \tau_{1}^{3}=120, \quad \sigma_{1} \tau_{1} \tau_{2}=72, & \tau_{1}^{4}=102, & \tau_{1}^{2} \tau_{2}=63, & \tau_{2}^{2}=45\end{array}$}} \\
\hline \multicolumn{3}{|c|}{$\sigma_{1} \sigma_{2} \tau_{1}=72, \quad \sigma_{2} \tau_{1}^{2}=72$} & & & & & \\
\hline$n_{d_{1}, d_{2}, 11}$ & $d_{1}=0$ & 1 & 2 & 3 & 4 & 5 & 6 \\
\hline$d_{2}=0$ & & 0 & 0 & 0 & 0 & 0 & 0 \\
\hline 1 & 0 & 960 & 1200 & 0 & 0 & 0 & 0 \\
\hline 2 & 0 & 420 & 20160 & 42300 & 600 & 0 & 0 \\
\hline 3 & 0 & 0 & 22800 & 668160 & 1867200 & 197520 & 0 \\
\hline 4 & 0 & 0 & 210 & 1206540 & 28032600 & 94238940 & 29975670 \\
\hline 5 & 0 & 0 & 0 & 91440 & 67122240 & 1368583200 & 5269901040 \\
\hline$n_{d_{1}, d_{2}, \sigma}$ & $d_{1}=0$ & 1 & 2 & 3 & 4 & 5 & 6 \\
\hline$d_{2}=0$ & & 0 & 0 & 0 & 0 & 0 & 0 \\
\hline 1 & 0 & 540 & 540 & 0 & 0 & 0 & 0 \\
\hline 2 & 0 & 270 & 11340 & 21330 & 270 & 0 & 0 \\
\hline 3 & 0 & 0 & 14580 & 382320 & 984960 & 95580 & 0 \\
\hline 4 & 0 & 0 & 135 & 756270 & 16113600 & 50752710 & 15091785 \\
\hline 5 & 0 & 0 & 0 & 58860 & 41366160 & 787977720 & 2873723940 \\
\hline$n_{d_{1}, d_{2}, 12}$ & $d_{1}=0$ & 1 & 2 & 3 & 4 & 5 & 6 \\
\hline$d_{2}=0$ & & 0 & 0 & 0 & 0 & 0 & 0 \\
\hline 1 & 0 & 960 & 720 & 0 & 0 & 0 & 0 \\
\hline 2 & 0 & 720 & 20160 & 31920 & 360 & 0 & 0 \\
\hline 3 & 0 & 0 & 31920 & 679680 & 1547760 & 137280 & 0 \\
\hline 4 & 0 & 0 & 360 & 1547760 & 28646400 & 81883440 & 22591320 \\
\hline 5 & 0 & 0 & 0 & 137280 & 81883440 & 1400849280 & 4715787120 \\
\hline
\end{tabular}

Table 8. Genus-0 invariants of determinantal 4-folds in (A.8) with $V=G(2,6)$. 
Open Access. This article is distributed under the terms of the Creative Commons Attribution License (CC-BY 4.0), which permits any use, distribution and reproduction in any medium, provided the original author(s) and source are credited.

\section{References}

[1] P. Candelas, G.T. Horowitz, A. Strominger and E. Witten, Vacuum Configurations for Superstrings, Nucl. Phys. B 258 (1985) 46 [INSPIRE].

[2] E. Witten, Phases of $N=2$ theories in two-dimensions, Nucl. Phys. B 403 (1993) 159 [hep-th/9301042] [INSPIRE].

[3] D.R. Morrison and M.R. Plesser, Summing the instantons: Quantum cohomology and mirror symmetry in toric varieties, Nucl. Phys. B 440 (1995) 279 [hep-th/9412236] [INSPIRE].

[4] K. Hori and C. Vafa, Mirror symmetry, hep-th/0002222 [InSPIRE].

[5] W. Gu and E. Sharpe, A proposal for nonabelian mirrors, arXiv:1806.04678 [INSPIRE].

[6] Z. Chen, W. Gu, H. Parsian and E. Sharpe, Two-dimensional supersymmetric gauge theories with exceptional gauge groups, arXiv: 1808.04070 [INSPIRE].

[7] V. Pestun et al., Localization techniques in quantum field theories, J. Phys. A 50 (2017) 440301 [arXiv:1608.02952] [InSPIRE].

[8] F. Benini and S. Cremonesi, Partition Functions of $\mathcal{N}=(2,2)$ Gauge Theories on $S^{2}$ and Vortices, Commun. Math. Phys. 334 (2015) 1483 [arXiv:1206.2356] [InSPIRE].

[9] N. Doroud, J. Gomis, B. Le Floch and S. Lee, Exact Results in D = 2 Supersymmetric Gauge Theories, JHEP 05 (2013) 093 [arXiv: 1206.2606] [INSPIRE].

[10] H. Jockers, V. Kumar, J.M. Lapan, D.R. Morrison and M. Romo, Two-Sphere Partition Functions and Gromov-Witten Invariants, Commun. Math. Phys. 325 (2014) 1139 [arXiv: 1208.6244] [INSPIRE].

[11] Y. Honma and M. Manabe, Exact Kähler Potential for Calabi-Yau Fourfolds, JHEP 05 (2013) 102 [arXiv:1302.3760] [INSPIRE].

[12] C. Closset, S. Cremonesi and D.S. Park, The equivariant A-twist and gauged linear $\sigma$-models on the two-sphere, JHEP 06 (2015) 076 [arXiv: 1504.06308] [INSPIRE].

[13] F. Benini and A. Zaffaroni, A topologically twisted index for three-dimensional supersymmetric theories, JHEP 07 (2015) 127 [arXiv: 1504.03698] [INSPIRE].

[14] K. Ueda and Y. Yoshida, Equivariant A-twisted GLSM and Gromov-Witten invariants of CY 3-folds in Grassmannians, JHEP 09 (2017) 128 [arXiv: 1602.02487] [INSPIRE].

[15] B. Kim, J. Oh, K. Ueda and Y. Yoshida, Residue mirror symmetry for Grassmannians, arXiv: 1607.08317 [INSPIRE].

[16] A. Gerhardus, H. Jockers and U. Ninad, The Geometry of Gauged Linear $\sigma$-model Correlation Functions, Nucl. Phys. B 933 (2018) 65 [arXiv:1803.10253] [INSPIRE].

[17] Y. Honma and M. Manabe, Local B-model Yukawa couplings from A-twisted correlators, PTEP 2018 (2018) 073A03 [arXiv: 1805.02661] [INSPIRE].

[18] H. Jockers, V. Kumar, J.M. Lapan, D.R. Morrison and M. Romo, Nonabelian 2D Gauge Theories for Determinantal Calabi-Yau Varieties, JHEP 11 (2012) 166 [arXiv:1205.3192] [INSPIRE]. 
[19] J. Harris, Graduate Texts in Mathematics. Vol. 133: Algebraic geometry, Springer-Verlag, New York U.S.A. (1992).

[20] W. Fulton, Ergeb. Math. Grenzgeb. (3). Vol. 2: Intersection theory, second edition, Springer-Verlag, Berlin Germany (1998).

[21] A. Givental, Homological geometry I. Projective hypersurfaces, Selecta Math. 1 (1995) 325.

[22] A. Givental, Equivariant Gromov-Witten Invariants, alg-geom/9603021.

[23] T. Coates and A. Givental, Quantum Riemann-Roch, Lefschetz and Serre, Ann. Math. 165 (2007) 15 [math/0110142] [INSPIRE].

[24] S. Hosono and H. Takagi, Mirror symmetry and projective geometry of Reye congruences I, J. Alg. Geom. 23 (2014) 279 [arXiv:1101.2746] [InSPIRE].

[25] M.-A. Bertin, Examples of Calabi-Yau 3-folds of $\mathbb{P}^{7}$ with $\rho=1$, Canad. J. Math. 61 (2009) $1050[\mathrm{math} / 0701511]$.

[26] D.A. Cox and S. Katz, Mathematical surveys and monographs. Vol. 68: Mirror symmetry and algebraic geometry, AMS, New York U.S.A. (1999).

[27] G. Bonelli, A. Sciarappa, A. Tanzini and P. Vasko, Vortex partition functions, wall crossing and equivariant Gromov-Witten invariants, Commun. Math. Phys. 333 (2015) 717 [arXiv: 1307.5997] [INSPIRE].

[28] S. Hosono, A. Klemm, S. Theisen and S.-T. Yau, Mirror symmetry, mirror map and applications to Calabi-Yau hypersurfaces, Commun. Math. Phys. 167 (1995) 301 [hep-th/9308122] [INSPIRE].

[29] S. Hosono, A. Klemm, S. Theisen and S.-T. Yau, Mirror symmetry, mirror map and applications to complete intersection Calabi-Yau spaces, Nucl. Phys. B 433 (1995) 501 [hep-th/9406055] [INSPIRE].

[30] B.R. Greene, D.R. Morrison and M.R. Plesser, Mirror manifolds in higher dimension, Commun. Math. Phys. 173 (1995) 559 [hep-th/9402119] [INSPIRE].

[31] A. Klemm and R. Pandharipande, Enumerative geometry of Calabi-Yau 4-folds, Commun. Math. Phys. 281 (2008) 621 [math/0702189] [INSPIRE].

[32] A. Popa and A. Zinger, Mirror symmetry for closed, open, and unoriented Gromov-Witten invariants, Adv. Math. 259 (2014) 448 [arXiv:1010.1946].

[33] R. Pandharipande and A. Zinger, Enumerative Geometry of Calabi-Yau 5-Folds, in New Developments in Algebraic Geometry, Integrable Systems and Mirror Symmetry, Kyoto Japan (2008), pg. 239 [arXiv:0802.1640].

[34] R. Gopakumar and C. Vafa, M theory and topological strings. 2., hep-th/9812127 [INSPIRE].

[35] Y. Cao, D. Maulik and Y. Toda, Genus zero Gopakumar-Vafa type invariants for Calabi-Yau 4-folds, Adv. Math. 338 (2018) 41 [arXiv:1801.02513] [INSPIRE].

[36] P. Candelas, X.C. De La Ossa, P.S. Green and L. Parkes, A Pair of Calabi-Yau manifolds as an exactly soluble superconformal theory, Nucl. Phys. B 359 (1991) 21 [INSPIRE].

[37] P.S. Aspinwall and D.R. Morrison, Topological field theory and rational curves, Commun. Math. Phys. 151 (1993) 245 [hep-th/9110048] [INSPIRE].

[38] K. Hori and D. Tong, Aspects of Non-Abelian Gauge Dynamics in Two-Dimensional $N=(2,2)$ Theories, JHEP 05 (2007) 079 [hep-th/0609032] [INSPIRE]. 
[39] D. Inoue, A. Ito and M. Miura, I-functions of Calabi-Yau 3-folds in Grassmannians, Commun. Num. Theor. Phys. 11 (2017) 273 [arXiv:1607.08137].

[40] S. Martin, Symplectic quotients by a nonAbelian group and by its maximal torus, Submitted to: Annals Math. (1999) [math/0001002] [INSPIRE].

[41] L.C. Mihalcea, Giambelli formulae for the equivariant quantum cohomology of the Grassmannian, Trans. AMS 360 (2008) 2285 [math/0506335].

[42] P. Griffiths and J. Harris, Principles of Algebraic Geometry, Wiley, New York U.S.A. (1978).

[43] V.V. Batyrev, I. Ciocan-Fontanine, B. Kim and D. van Straten, Conifold Transitions and Mirror Symmetry for Calabi-Yau Complete Intersections in Grassmannians, Nucl. Phys. B 514 (1998) 640 [alg-geom/9710022].

[44] B. Haghighat and A. Klemm, Topological Strings on Grassmannian Calabi-Yau manifolds, JHEP 01 (2009) 029 [arXiv: 0802.2908] [INSPIRE].

[45] A. Gerhardus and H. Jockers, Quantum periods of Calabi-Yau fourfolds, Nucl. Phys. B 913 (2016) 425 [arXiv: 1604.05325] [INSPIRE].

[46] B. Jia, E. Sharpe and R. Wu, Notes on nonabelian $(0,2)$ theories and dualities, JHEP 08 (2014) 017 [arXiv:1401.1511] [INSPIRE].

[47] A.-M. Li and Y. Ruan, Symplectic surgery and Gromov-Witten invariants of Calabi-Yau 3-folds, Invent. Math. 145 (2001) 151 [math/9803036].

[48] C. Schoen, On the geometry of a special determinantal hypersurface associated to the Mumford-Horrocks vector bundle, J. Reine Angew. Math. 364 (1986) 85.

[49] M. Gross and S. Popescu, Calabi-Yau threefolds and moduli of Abelian surfaces. 1., math/0001089 [INSPIRE].

[50] T.H. Gulliksen and O.G. Negård, Un complexe résolvant pour certains idéaux déterminantiels, C.R. Acad. Sci. Paris Sér. A-B 274 (1972) A16.

[51] M. Kapustka and G. Kapustka, A cascade of determinantal Calabi-Yau threefolds, Math. Nachr. 283 (2010) 1795 [arXiv:0802.3669].

[52] A. Caldararu, J. Knapp and E. Sharpe, GLSM realizations of maps and intersections of Grassmannians and Pfaffians, JHEP 04 (2018) 119 [arXiv:1711.00047] [INSPIRE].

[53] A. Kanazawa, Pfaffian Calabi-Yau threefolds and mirror symmetry, Commun. Num. Theor. Phys. 6 (2012) 661 [INSPIRE].

[54] T. Hübsch, Calabi-Yau manifolds: A Bestiary for physicists, World Scientific, Singapore (1992).

[55] O. Küchle, On Fano 4-folds of index 1 and homogeneous vector bundles over Grassmannians, Math. Z. 218 (1995) 563.

[56] L.B. Anderson, Heterotic and M-theory Compactifications for String Phenomenology, Ph.D. Thesis, Oxford University, Oxford U.K. (2008) [arXiv: 0808.3621] [INSPIRE].

[57] R. Blumenhagen, B. Jurke, T. Rahn and H. Roschy, Cohomology of Line Bundles: A Computational Algorithm, J. Math. Phys. 51 (2010) 103525 [arXiv: 1003.5217] [INSPIRE].

[58] R. Blumenhagen, B. Jurke, T. Rahn and H. Roschy, Cohomology of Line Bundles: Applications, J. Math. Phys. 53 (2012) 012302 [arXiv: 1010.3717] [InSPIRE]. 
[59] A. Klemm, B. Lian, S.S. Roan and S.-T. Yau, Calabi-Yau fourfolds for M-theory and F-theory compactifications, Nucl. Phys. B 518 (1998) 515 [hep-th/9701023] [INSPIRE].

[60] D. Inoue, A. Ito and M. Miura, Complete intersection Calabi-Yau manifolds with respect to homogeneous vector bundles on Grassmannians, arXiv:1607.07821.

[61] V. Benedetti, Manifolds of low dimension with trivial canonical bundle in Grassmannians, arXiv: 1609.02695.

[62] D. Maulik, R. Pandharipande, R.P. Thomas and A. Pixton, Curves on K3 surfaces and modular forms, J. Topol. 3 (2010) 937 [arXiv: 1001.2719] [INSPIRE]. 